\title{
Self-interaction correction to density-functional approximations for many-electron systems
}

\author{
J. P. Perdew \\ Department of Physics and Quantum Theory Group, Tulane University, New Orleans, Louisiana 70118 \\ Alex Zunger \\ Solar Energy Research Institute, Golden, Colorado 80401 \\ and Department of Physics, University of Colorado, Boulder, Colorado 80302 \\ (Received 31 October 1980)
}

\begin{abstract}
The exact density functional for the ground-state energy is strictly self-interaction-free (i.e., orbitals demonstrably do not self-interact), but many approximations to it, including the local-spin-density (LSD) approximation for exchange and correlation, are not. We present two related methods for the self-interaction correction (SIC) of any density functional for the energy; correction of the self-consistent one-electron potenial follows naturally from the variational principle. Both methods are sanctioned by the Hohenberg-Kohn theorem. Although the first method introduces an orbital-dependent single-particle potential, the second involves a local potential as in the Kohn-Sham scheme. We apply the first method to LSD and show that it properly conserves the number content of the exchangecorrelation hole, while substantially improving the description of its shape. We apply this method to a number of physical problems, where the uncorrected LSD approach produces systematic errors. We find systematic improvements, qualitative as well as quantitative, from this simple correction. Benefits of SIC in atomic calculations include (i) improved values for the total energy and for the separate exchange and correlation pieces of it, (ii) accurate binding energies of negative ions, which are wrongly unstable in LSD, (iii) more accurate electron densities, (iv) orbital eigenvalues that closely approximate physical removal energies, including relaxation, and (v) correct longrange behavior of the potential and density. It appears that SIC can also remedy the LSD underestimate of the band gaps in insulators (as shown by numerical calculations for the rare-gas solids and $\mathrm{CuCl}$ ), and the LSD overestimate of the cohesive energies of transition metals. The LSD spin splitting in atomic $\mathrm{Ni}$ and $s-d$ interconfigurational energies of transition elements are almost unchanged by SIC. We also discuss the admissibility of fractional occupation numbers, and present a parametrization of the electron-gas correlation energy at any density, based on the recent results of Ceperley and Alder.
\end{abstract}

\section{INTRODUCTION}

A major problem of solid-state theory and quantum chemistry is to understand the behavior of many electrons interacting via Coulomb's law:

$$
\hat{V}_{e e}=\frac{1}{2} \sum_{i} \sum_{j \neq i} \frac{1}{\left|\overrightarrow{\mathrm{r}}_{i}-\overrightarrow{\mathrm{r}}_{j}\right|}
$$

(All equations are in atomic units, $\hbar=m=e^{2}=1$.) In the earliest quantum-mechanical theory, Thomas and Fermi replaced the expectation value $\left\langle\hat{V}_{e e}\right\rangle$ by the direct Coulomb energy, a functional of the electron number density $n(\overrightarrow{\mathrm{r}})$ :

$$
U[n]=\frac{1}{2} \int d^{3} r \int d^{3} r^{\prime} \frac{n(\overrightarrow{\mathrm{r}}) n\left(\overrightarrow{\mathrm{r}}^{\prime}\right)}{\left|\overrightarrow{\mathrm{r}}-\overrightarrow{\mathrm{r}}^{\prime}\right|} .
$$

As early as 1934, Fermi and Amaldi ${ }^{1}$ observed the failure of Eq. (2) to vanish for one-electron systems due to the spurious self-interaction inherent in it, and proposed the first and crudest version of self-interaction correction:

$$
\left\langle\hat{V}_{e e}\right\rangle=U[n]-N U\left[\frac{n}{N}\right],
$$

where $N$ is the number of electrons in the system. The mean-field Hartree-Fock ( $\mathrm{HF}$ ) approximation introduced orbitals $\psi_{\alpha \sigma}(\overrightarrow{\mathrm{r}})$ with occupation numbers $f_{\alpha \sigma}$ obeying Fermi statistics:

$$
n(\overrightarrow{\mathrm{r}})=\sum_{\alpha \sigma} n_{\alpha \sigma}(\overrightarrow{\mathrm{r}}),
$$

where

$$
n_{\alpha \sigma}(\vec{r})=f_{\alpha \sigma}\left|\psi_{\alpha \sigma}(\vec{r})\right|^{2}
$$

is the density of an orbital with quantum numbers $\alpha$ and $\sigma$, and $\sigma=+\frac{1}{2}(\uparrow)$ or $-\frac{1}{2}(\downarrow)$ is the spin. In this approximation, the total interelectronic energy is given as a sum of direct and exchange contributions:

$$
\left\langle\hat{V}_{e e}\right\rangle=U[n]+E_{x},
$$

where the exchange energy is

$E_{x}=-\frac{1}{2} \sum_{\sigma} \sum_{\alpha, \alpha^{\prime}} f_{\alpha \sigma} f_{\alpha^{\prime} \sigma} \int d^{3} r \int d^{3} \gamma^{\prime} \frac{\psi_{\alpha \sigma}^{*}(\overrightarrow{\mathrm{r}}) \psi_{\alpha^{\prime} \sigma}^{*}\left(\overrightarrow{\mathbf{r}}^{\prime}\right) \psi_{\alpha^{\prime} \sigma}(\overrightarrow{\mathrm{r}}) \psi_{\alpha \sigma}\left(\overrightarrow{\mathbf{r}}^{\prime}\right)}{\left|\overrightarrow{\mathbf{r}}-\overrightarrow{\mathbf{r}}^{\prime}\right|}$.

The $\alpha^{\prime}=\alpha$ terms in Eq. (7) (the only ones retained in the Hartree approximation) constitute a selfexchange energy:

$$
\text { self-exchange }=-\sum_{\alpha \sigma} U\left[n_{\alpha_{\sigma}}\right]
$$

which correctly cancels the self-Coulomb energy in $U[n]$ on an orbital-by-orbital basis. Table I (Ref. 2) shows how much of the exchange energy in atoms is simply self-exchange.

Although the $\mathrm{HF}$ approximation produces only a 
TABLE I. Hartree-Fock total energy, exchange energy, and interelectronic exchange energy (which omits self-exchange) for lighter inert gas atoms (from Ref. 2). The correlation energy is from Ref. 62. Energies in eV.

\begin{tabular}{lrrrrr}
\hline \hline Atom & $\boldsymbol{Z}$ & Total energy & Exchange & $\begin{array}{c}\text { Interelectronic } \\
\text { exchange }\end{array}$ & Correlation \\
\hline $\mathrm{He}$ & 2 & -77.9 & -27.9 & 0.0 & -1.1 \\
$\mathrm{Ne}$ & 10 & -3497.8 & -329.5 & -60.3 & -10.4 \\
$\mathrm{Ar}$ & 18 & -14334.7 & -821.3 & -203.5 & -19.9 \\
$\mathrm{Kr}$ & 36 & -74883.4 & -2561.9 & -908.3 & \\
\hline \hline
\end{tabular}

very small relative error in the total energy of atoms, it is not suitable for most solid-state and many molecular applications. The total energy is dominated by high-density inner-shell electrons that are well described by Hartree-Fock theory, but one is more usually interested in the low-density valence electrons, for which correlation (neglected in HF theory) may be as important as exchange. In addition, the long range of the Coulomb interaction produces unrealistic features in the HF energy eigenvalues, e.g., vanishing density of states at the Fermi level in metals, ${ }^{3}$ unphysically large band gaps in insulators (sometimes by a factor of 2-5), ${ }^{4}$ etc. While correlation effects can be addressed through configuration-interaction corrections to $\mathrm{HF}$, the complexity of these corrections, their remarkable sensitivity to the choice of basis functions, and the increase in effort required with the decrease in spacing between energy levels, preclude application to large systems.

The density-functional theory ${ }^{5-8}$ provides an alternative to this approach. This theory which is exact in principle includes correlation explicitly. in the total energy and one-body potential; typically in practice exchange and correlation are treated together in the local-spin-density (LSD) approximation. ${ }^{7,8}$ This essentially statistical approximation works fairly well even for one-electron systems ${ }^{9}:$ In the hydrogen atom, the direct (self-interacting) Coulomb energy $U[n]$ is large $(8.5 \mathrm{eV})$, but about $93 \%$ of this spurious energy is cancelled by the LSD exchange-correlation energy. Unlike HF theory where the self-interaction is completely canceled because the direct electrostatic and exchange operators have the same kernel $1 /\left|\overrightarrow{\mathbf{r}}-\overrightarrow{\mathbf{r}}^{\prime}\right|$, LSD achieves only a partial cancellation, assisted largely by spin-polarization and correlation effects. Hence, in LSD a residue of spurious self-interaction remains as the price to be paid for a simple, local one-electron potential. Clearly, this self-interaction vanishes for orbitals delocalized over extended systems. We will see, however, that self-interaction leads to a number of systematic errors for finite systems and localized states in extended systems.
The quantitative successes of the LSD approximation have been impressive, particularly for molecular bonding, ${ }^{10,11}$ metallic magnetism, 12-14 cohesion, ${ }^{12,15}$ and the surface electronic properties of metals ${ }^{16,17}$ and semi-conductors. ${ }^{18-20}$ Nevertheless, there remain some worrisome failures of LSD, some of which have been revealed only recently. These include the facts that:

(i) While the LSD total energy of a metal surface is too low when compared with the exact value, ${ }^{21,22}$ the LSD energy for atoms is too high. ${ }^{23}$ Furthermore, the lowest-order correction to the LSD exchange-correlation energy predicted by many-body theory, i.e., the density-gradient correction, ${ }^{24}$ is positive and so its inclusion can only worsen the agreement between the calculated energies of atoms and experiment.

(ii) For atom $\mathrm{s}^{23}$ the magnitude of the exchange is consistently underestimated by $10-15 \%$ in LSD. The magnitude of the correlation energy is overestimated by as much as 100-200\%.

(iii) The experimentally stable negative ions (e.g., $\mathrm{H}^{-}, \mathrm{O}^{-}, \mathrm{F}^{-}$) are predicted to be unstable in LSD. ${ }^{25,26}$

(iv) Self-consistent LSD band-structure calculations systematically underestimate the one-electron energy gaps of insulators by as much as $40 \%$ (e.g., Refs. 27-31). More generally, the LSD oneelectron energy eigenvalues are not close to physical removal energies from bound states. There are often large deviations from experiment when LSD energy eigenvalues are identified with the positions of surface states, deep defect levels in solids, core bands in solids, etc.

(v) The long-range behavior of the LSD one-electron potential for ions of charge $Q$ is $-Q / r$, rather than the electrostatically correct limit of $-(Q+1) /$ $r$. Among other problems, this leads to an erroneous description of charged point-defect states in solids.

(vi) The LSD calculated spin splitting of the energy bands in ferromagnetic metals [e.g., Ni (Ref. 32)] is often much larger than the observed value.

(vii) LSD total energies unduly favor the $d^{n-1} s^{1}$ configuration over the $d^{n-2} s^{2}$ configuration in $3 d$ 
transition atoms..$^{33}$ In addition, the LSD ordering of $s$ and $d$ levels in the $d^{n-1} s^{1}$ configuration of the $3 d$ elements Sc to $\mathrm{Mn}$ is reversed relative to HF.

Much of the criticism of the LSD approximation has been directed toward its incomplete description of many-body correlation effects. In this paper we show that many of the problems indicated above are interrelated and that a simple, physically transparent one-body correction to LSDthe subtraction of spurious self-interaction-accounts for and corrects most (but not all) of the qualitative and quantitative discrepancies. Empirical methods used previously for correcting some of these discrepancies (e.g., use of adjustable exchange coefficient $\alpha$, empirical gradient terms, etc.) become unnecessary. A preliminary version of our work has already appeared. ${ }^{34}$

\section{FORMALISM}

\section{A. Spin-density-functional theory}

We begin with a review of spin-density-functional theory, ${ }^{6-8}$ including a synthesis of recent developments, following the simple and elegant approach of Levy. ${ }^{35}$ This formally exact theory will then serve to introduce self-interaction corrections in Sec. II B.

Consider a system of $N$ electrons with the Hamiltonian

$$
\hat{H}=\hat{T}+\hat{V}_{e e}+\sum_{i} v\left(\overrightarrow{\mathbf{r}}_{i}\right)-2 \mu \sum_{i} B\left(\vec{\gamma}_{i}\right) \hat{s}_{z}^{i},
$$

where $v(\overrightarrow{\mathrm{r}})$ and $B(\overrightarrow{\mathrm{r}})$ are external scalar and magnetic fields (the latter coupling to electron spin $\left.\hat{s}_{z}^{i}\right)$, and $\hat{T}$ is the kinetic energy operator. Let $n_{\uparrow}(\overrightarrow{\mathrm{r}})$ and $n_{\downarrow}(\overrightarrow{\mathrm{r}})$ be up- and down-spin number densities, and define the universal functional ${ }^{35}$

$$
Q\left[n_{\uparrow}, n_{\downarrow}\right]=\min \left\langle\hat{T}+\hat{V}_{e e}\right\rangle,
$$

which searches the set of all $N$-particle antisymmetric wave functions producing the given spin densities $n_{\sigma}(\overrightarrow{\mathrm{r}})=\left\langle\hat{n}_{\sigma}(\overrightarrow{\mathrm{r}})\right\rangle$, and delivers the minimum expectation value. Then the usual variational principle $(\langle\hat{H}\rangle \geqslant$ the ground-state energy $E)$ implies that for any choice of trial spin densities $n_{\uparrow}$ and $n_{\downarrow}$ representing $N$ electrons,

$Q\left[n_{\uparrow}, n_{\downarrow}\right]+\int d^{3} r v(\overrightarrow{\mathrm{r}}) n(\overrightarrow{\mathrm{r}})-2 \mu \int d^{3} r B(\overrightarrow{\mathrm{r}}) \sum_{\sigma} \sigma n_{\sigma}(\overrightarrow{\mathrm{r}}) \geqslant E$,

where $n=\sum_{\sigma} n_{\sigma}$. Furthermore, the true groundstate spin densities are those which make Eq. (11) an equality. (This is the generalized Hohenberg$\mathrm{Kohn}^{6}$ theorem. Note that the argument does not require a nondegenerate ground state, nor does it require that the trial spin densities be $v$ representable.)

The variational principle (11) may be used to calculate $n_{\uparrow}, n_{\downarrow}$, and $E$, if the functional dependence of $Q$ on $n_{\uparrow}$ and $n_{\downarrow}$ is known. It is convenient to break $Q$ up into three parts as follows:

$$
Q\left[n_{\uparrow}, n_{\downarrow}\right]=T\left[n_{\uparrow}, n_{\downarrow}\right]+U[n]+E_{\text {xc }}\left[n_{\uparrow}, n_{\downarrow}\right],
$$

where $T\left[n_{\uparrow}, n_{\downarrow}\right]$ is the "noninteracting" kinetic energy defined below, $U[n]$ is the direct Coulomb energy of Eq. (2), and $E_{\mathrm{xc}}\left[n_{\uparrow}, n_{\downarrow}\right]$ is the exchangecorrelation energy.

The spin densities can always be written (nonuniquely) in terms of orthonormal orbitals $\psi_{\alpha \sigma}(\overrightarrow{\mathrm{r}})$ and occupation numbers in the interval $0 \leqslant f_{\alpha \sigma} \leqslant 1$ :

$$
n_{\sigma}(\overrightarrow{\mathbf{r}})=\sum_{\alpha} f_{\alpha \sigma}\left|\psi_{\alpha \sigma}(\overrightarrow{\mathbf{r}})\right|^{2}
$$

We define $e^{36}$

$$
T\left[n_{\uparrow}, n_{\downarrow}\right]=\min \left(\sum_{\alpha \sigma} f_{\alpha \sigma}\left\langle\psi_{\alpha \sigma}\left|-\frac{1}{2} \nabla^{2}\right| \psi_{\alpha \sigma}\right\rangle\right),
$$

where the minimum is over all possible $f_{\alpha \sigma}$ and $\psi_{\alpha \sigma}$ yielding the given spin densities. (For further discussion of this definition, see Appendix A.) Following Janak, ${ }^{37}$ the minimization of the lefthand side of Eq. (11) can now be accomplished in two steps:

(1) Assume a fixed set of $f_{\alpha \sigma}$ and minimize

$$
\tilde{E}=\sum_{\alpha \sigma} f_{\alpha \sigma}\left\langle\psi_{\alpha \sigma}\left|-\frac{1}{2} \nabla^{2}\right| \psi_{\alpha \sigma}\right\rangle+U[n]+E_{\mathrm{xc}}\left[n_{\uparrow}, n_{\uparrow}\right]+\int d^{3} \gamma v(\overrightarrow{\mathrm{r}}) n(\overrightarrow{\mathrm{r}})-2 \mu \int d^{3} \gamma B(\overrightarrow{\mathrm{r}}) \sum_{\sigma} \sigma n_{\sigma}(\overrightarrow{\mathrm{r}})
$$

[with $n_{\sigma}$ given by Eq. (13)] with respect to the $\psi_{\alpha \sigma}(\overrightarrow{\mathrm{r}})$, subject to the normalization constraint on the orbitals. The resulting Euler equation is

$\frac{\delta}{\delta \psi_{\alpha \sigma}(\overrightarrow{\mathrm{r}})}\left(\tilde{E}-\sum_{\alpha^{\prime} \sigma^{\prime}} \epsilon_{\alpha^{\prime} \sigma^{\prime}} f_{\alpha^{\prime} \sigma^{\prime}} \int d^{3} r^{\prime}\left|\psi_{\alpha^{\prime} \sigma}\left(\overrightarrow{\mathbf{r}}^{\prime}\right)\right|^{2}\right)=0$,

where $\epsilon_{\alpha \sigma} f_{\alpha \sigma}$ is a Lagrange multiplier. This leads directly to the Kohn-Sham ${ }^{7}$ one-electron self-consistent equations:

$$
\left[-\frac{1}{2} \nabla^{2}+v_{\text {eff }}^{\sigma}(\overrightarrow{\mathrm{r}})\right] \psi_{\alpha \sigma}(\overrightarrow{\mathrm{r}})=\epsilon_{\alpha \sigma} \psi_{\alpha \sigma}(\overrightarrow{\mathrm{r}}),
$$

with an effective one-body potential

$$
\begin{aligned}
v_{\text {eff }}^{\sigma}(\overrightarrow{\mathrm{r}})= & v(\overrightarrow{\mathrm{r}})-2 \mu \sigma B(\overrightarrow{\mathrm{r}})+u([n] ; \overrightarrow{\mathrm{r}}) \\
& +v_{\mathrm{xc}}^{\sigma}\left(\left[n_{\uparrow}, n_{\downarrow}\right] ; \overrightarrow{\mathrm{r}}\right),
\end{aligned}
$$

where the direct Coulomb potential is

$$
u([n] ; \overrightarrow{\mathbf{r}})=\frac{\delta}{\delta n(\overrightarrow{\mathbf{r}})} U[n]=\int d^{3} r^{\prime} \frac{n\left(\overrightarrow{\mathbf{r}}^{\prime}\right)}{\left|\overrightarrow{\mathbf{r}}-\overrightarrow{\mathbf{r}}^{\prime}\right|},
$$


and the exchange-correlation potential is

$$
v_{\mathrm{xc}}^{\sigma}\left(\left[n_{\uparrow}, n_{\downarrow}\right] ; \overrightarrow{\mathrm{r}}\right)=\frac{\delta}{\delta n_{\sigma}(\overrightarrow{\mathrm{r}})} E_{\mathrm{xc}}\left[n_{\uparrow}, n_{\uparrow}\right]
$$

The significance of the eigenvalue $\epsilon_{\alpha \sigma}$ can be seen by an argument of Slater ${ }^{5}$ and Janak ${ }^{37}$ : Differentiation of Eq. (15) with respect to the occupation number $f_{\alpha \sigma}$ (with all orbitals fixed) yields

$$
\begin{aligned}
\frac{\partial \tilde{E}}{\partial f_{\alpha \sigma}} & =\int d^{3} r \psi_{\alpha \sigma}^{*}(\overrightarrow{\mathrm{r}})\left[-\frac{1}{2} \nabla^{2}+v_{\mathrm{eff}}^{\sigma}(\overrightarrow{\mathrm{r}})\right] \psi_{\alpha \sigma}(\overrightarrow{\mathrm{r}}) \\
& =\epsilon_{\alpha \sigma} .
\end{aligned}
$$

Since $\tilde{E}$ has been minimized with respect to normalization-preserving variations of the orbitals, it follows that in any infinitesimal, normalizationpreserving variation of the occupation numbers and orbitals,

$$
\delta \tilde{E}=\sum_{\alpha \sigma} \epsilon_{\alpha \sigma} \delta f_{\alpha \sigma} .
$$

(2) Now minimize $\tilde{E}$ with respect to the $f_{\alpha \sigma}$, subject to the particle-conserving constraint $\sum_{\alpha \sigma}^{\alpha \sigma} f_{\alpha \sigma}$ $=N$. This is equivalent to the unconstrained ${ }^{38} \mathrm{~min}$ imization of $\tilde{E}-\mu N$, where $\mu$ is the chemical potential $[=- \text { electronegativity }]^{39}$ :

$$
\delta(\tilde{E}-\mu N)=\sum_{\alpha \sigma}\left(\epsilon_{\alpha \sigma}-\mu\right) \delta f_{\alpha \sigma} \geqslant 0 .
$$

From Eq. (23) we immediately find an "aufbau principle" ${ }^{5,37}$ : orbitals with $\epsilon_{\alpha_{\sigma}}<\mu$ have $f_{\alpha \sigma}=1$, and those with $\epsilon_{\alpha \sigma}>\mu$ have $f_{\alpha \sigma}=0$. Fractional occupation is found only for $\epsilon_{\alpha \sigma}=\mu$. If it turns out that all occupation numbers are 0 or 1 , then the ground-state spin densities of the real system are also ground-state spin densities of a fictitious system of noninteracting electrons moving in the potential $v_{\text {eff }}^{\sigma}(\overrightarrow{\mathrm{r}})$, as was assumed in the original Kohn-Sham ${ }^{7}$ derivation of Eq. (17). This assumption may not be universally true, and in the present formulation it is unnecessary. If this assumption is true, and if in addition the interacting ground state is adiabatically connected to the noninteracting one, then the exchange-correlation energy may be written as half the sum of the electrostatic interaction of each electron with its positively charged exchange-correlation hole $e^{21,40}$ :

$$
E_{\mathrm{xc}}=\frac{1}{2} \int d^{3} r \int d^{3} r^{\prime} \frac{n(\overrightarrow{\mathrm{r}}) \rho\left(\overrightarrow{\mathrm{r}}, \overrightarrow{\mathrm{r}}^{\prime}\right)}{\left|\overrightarrow{\mathbf{r}}-\overrightarrow{\mathrm{r}}^{\prime}\right|}
$$

where

$$
\begin{aligned}
\rho\left(\overrightarrow{\mathbf{r}}, \overrightarrow{\mathbf{r}}^{\prime}\right)=\int_{0}^{1} d \lambda & \left\langle\Psi_{\lambda}\right|[\hat{n}(\overrightarrow{\mathbf{r}})-n(\overrightarrow{\mathbf{r}})] \\
& \times\left[\hat{n}\left(\overrightarrow{\mathbf{r}}^{\prime}\right)-n\left(\overrightarrow{\mathbf{r}}^{\prime}\right)\right] / n(\overrightarrow{\mathrm{r}})-\delta\left(\overrightarrow{\mathbf{r}}-\overrightarrow{\mathbf{r}}^{\prime}\right)\left|\Psi_{\lambda}\right\rangle
\end{aligned}
$$

is the density at $\overrightarrow{\mathbf{r}}^{\prime}$ of the hole around an electron at $\vec{r} . \Psi_{\lambda}$ is the ground state of a fictitious system in which the electron-electron interaction is $\lambda /\left|\overrightarrow{\mathbf{r}}-\overrightarrow{\mathbf{r}}^{\prime}\right|$ and the spin densities are those of the real system $(\lambda=1)$. The $\lambda$ integration appears because of the definitions of $T\left[n_{\uparrow}, n_{\downarrow}\right](\neq\langle\hat{T}\rangle)$ and $U[n]$ $+E_{\mathrm{xc}}\left[n_{\uparrow}, n_{\downarrow}\right]\left(\neq\left\langle\hat{V}_{e e}\right\rangle\right)$; the integrand of Eq. (25) at the limit $\lambda=0$ is the exchange hole. From the definition (25), one gets

$$
\int d^{3} r^{\prime} \rho\left(\overrightarrow{\mathrm{r}}, \overrightarrow{\mathrm{r}}^{\prime}\right)=-1
$$

i.e., the electron and its hole together constitute a neutral object.

Spin-density-functional calculations would yield exact results if the exact $E_{\text {xc }}\left[n_{\uparrow}, n_{\downarrow}\right]$ were known and used. In practice one often makes the localspin-density (LSD) approximation ${ }^{7,8}$

$$
E_{\mathrm{xc}}^{\mathrm{LSD}}=\int d^{3} r n(\overrightarrow{\mathrm{r}}) \epsilon_{\mathrm{xc}}\left(n_{\uparrow}(\overrightarrow{\mathrm{r}}), n_{\downarrow}(\overrightarrow{\mathrm{r}})\right),
$$

where $\epsilon_{\mathrm{xc}}\left(n_{\uparrow}, n_{\downarrow}\right)$ is the exchange-correlation energy per particle of an electron gas with uniform spin densities $n_{\uparrow}, n_{\downarrow}$. In this way the results of manybody calculations for the homogeneous system, which have recently become quite reliable, ${ }^{41,42}$ can be folded into calculations for inhomogeneous systems. The functional derivative $(20)$ gives the LSD exchange-correlation potential

$$
v_{\mathrm{xc}}^{\sigma, \mathrm{LSD}}(\overrightarrow{\mathrm{r}})=\mu_{\mathrm{xc}}^{\sigma}\left(n_{\uparrow}(\overrightarrow{\mathrm{r}}), n_{\downarrow}(\overrightarrow{\mathrm{r}})\right),
$$

where $\mu_{x c}^{\sigma}\left(n_{\uparrow}, n_{\downarrow}\right)=\partial\left[n \epsilon_{x c}\left(n_{\uparrow}, n_{\downarrow}\right)\right] / \partial n_{\sigma}$.

By construction the LSD approximation becomes exact when the spin densities vary slowly enough on the scale of the local Fermi wavelength and screening length, a formal validity condition that is seldom satisfied in real systems. For reasons reviewed in Sec. II C, the LSD approximation is still reasonably successful for real systems, while inclusion of the next term in the density-gradient expansion does not give the systematic improvement that might naively be expected.

\section{B. Self-interaction correction}

From our definitions of the functionals $Q, T$, and $E_{\mathrm{xc}}$, it follows at once that the exchange-correlation energy of a single, fully occupied orbital must exactly cancel its self-direct Coulomb energy, i.e.,

$$
U\left[n_{\alpha \sigma}\right]+E_{\mathrm{xc}}\left[n_{\alpha \sigma}, 0\right]=0,
$$

where $n_{\alpha \sigma}=f_{\alpha \sigma}\left|\psi_{\alpha \sigma}\right|^{2}$ and $f_{\alpha \sigma}=1$. (Note that a single orbital is fully spin polarized, and note further that the orbital density $n_{\alpha \sigma}$ does not have to be a possible ground-state density.) Although Eq. (29) is hardly surprising, this is the first proof of it to our knowledge. By a suitable continuation 
of $E_{\mathrm{xc}}$ to noninteger numbers of electrons, we can also satisfy Eq. (29) for $f_{\alpha \sigma}<1$. (Physical reasons for doing so are presented in Appendix B.) Further insight can be gained if we define the exchange energy $E_{x}\left[n_{\uparrow}, n_{\downarrow}\right]$ as the right-hand side of Eq. (7), which is a functional of the spin densities because the Kohn-Sham orbitals and occupation numbers are. (This definition is obviously the correct one for a single, fully occupied orbital.) Then we find directly from the definitions of $U$ and $E_{x}$ that

$$
U\left[n_{\alpha \sigma}\right]+E_{x}\left[n_{\alpha \sigma}, 0\right]=0 .
$$

Defining the correlation energy as $E_{c}=E_{x_{c}}-E_{x}$, we find from Eqs. (29) and (30) that

$$
E_{c}\left[n_{\alpha \sigma}\right]=0 \text {. }
$$

Equations (29), (30), and (31) form the basis for our self-interaction corrections.

Suppose now that we have some approximation $E_{\text {xc }}^{\text {appox }}\left[n_{\uparrow}, n_{\downarrow}\right]$ (such as LSD, the gradient expansion, pseudopotential LSD screening, etc.) which does not satisfy Eq. (29) but is useful otherwise. We define our self-interaction corrected (SIC) version of this approximation by

$$
E_{\mathrm{xc}}^{\mathrm{SIC}}=E_{\mathrm{xc}}^{\mathrm{approx}}\left[n_{\uparrow}, n_{\downarrow}\right]-\sum_{\alpha \sigma} \delta_{\alpha \sigma},
$$

where

$$
\delta_{\alpha \sigma}=U\left[n_{\alpha \sigma}\right]+E_{\text {xc }}^{\text {approx }}\left[n_{\alpha \sigma}, 0\right]
$$

is the self-interaction of orbital $\alpha \sigma$. Unlike some other schemes which have been previously proposed (Sec. IV), this correction may be applied to any spin-density functional for $E_{\mathrm{xc}}$; when applied to the exact one the correction vanishes.

In order to get some feeling for the behavior and magnitude of the self-interaction correction, we pause to estimate it within LSD. Gadre et al..$^{43}$ have proved the general inequality [for $n(\overrightarrow{\mathrm{r}}) \geqslant 0$ and $\left.N=\int d^{3} r n(\overrightarrow{\mathrm{r}})\right]$ :

$$
U[n] \leqslant 1.092 N^{2 / 3} \int d^{3} r n^{4 / 3}(\overrightarrow{\mathbf{r}}) .
$$

Since the LSD approximation for exchange is

$$
E_{x}^{\mathrm{LSD}}\left[n_{\uparrow}, n_{\downarrow}\right]=-0.9305 \int d^{3} r\left[n_{\uparrow}^{4 / 3}(\overrightarrow{\mathrm{r}})+n_{\downarrow}^{4 / 3}(\overrightarrow{\mathrm{r}})\right],
$$

the rough cancellation between $U\left[n_{\alpha \sigma}\right]$ and $E_{x}^{\text {LSD }}\left[n_{\alpha_{\sigma}}, 0\right]$ for a single occupied orbital is evident. The spurious residue of this incomplete cancellation may, however, be non-negligible.

$$
\delta_{\alpha \sigma} \leqslant 0.16 \int d^{3} r n_{\alpha \sigma}^{4 / 3}(\overrightarrow{\mathrm{r}}) .
$$

Since (34) is usually close to an equality, ${ }^{43}$ so is (36). We see that, in the absence of correlation, $\delta_{\alpha \sigma}$ is positive and the self-interaction correction to the LSD total energy [Eq. (32)] is negative. Furthermore, the correction becomes more significant as the localization of the orbital increases: For a $1 s$ hydrogenic orbital, $n_{\alpha \sigma}(\overrightarrow{\mathrm{r}})=Z^{3} e^{-2 Z r} / \pi$ and Eq. (36) becomes $\delta_{\alpha \sigma} \leqslant 0.047 Z$; as $Z$ increases, correlation becomes less important relative to exchange and the inequality gets closer to a relative equality. On the other hand, for an electron delocalized over a volume $\Omega, n_{\alpha \sigma}$ is of order $\Omega^{-1}$ so $\delta_{\alpha \sigma}$ is of order $\Omega^{-1 / 3}$, which vanishes as $\Omega \rightarrow \infty$. (In a metal the sum over orbitals contributes another factor of $\Omega$, so the self-interaction correction to the total energy is of order $\Omega^{2 / 3}$.) It follows that, for densities varying slowly enough over space, the LSD and self-interaction-corrected LSD (SIC-LSD) approximations both yield the exact energy per electron.

In order to have a self-consistent self-interaction correction, we now seek the orbitals $\bar{\psi}_{\alpha \sigma}$ and occupation numbers $\bar{f}_{\alpha \sigma}$ which minimize $\tilde{E}$ of Eq. (15), with $E_{\mathrm{xc}}$ replaced by $E_{\mathrm{xc}}^{\text {SIC }}$. (Here we use a bar to denote quantities which are self-consistent with a self-interaction-corrected potential; elsewhere our notation will not be so explicit.) Following the procedure of Eq. (16), we obtain a oneelectron Schrödinger equation like Eq. (17),

$$
\left[-\frac{1}{2} \nabla^{2}+\bar{v}_{\text {eff }}^{\alpha \sigma}(\overrightarrow{\mathrm{r}})\right] \bar{\psi}_{\alpha \sigma}(\overrightarrow{\mathrm{r}})=\epsilon_{\alpha \sigma}^{\mathrm{SIC}} \bar{\psi}_{\alpha \sigma}(\overrightarrow{\mathrm{r}}),
$$

but with an orbital-dependent potential:

$$
\bar{v}_{\text {eff }}^{\alpha \sigma}(\overrightarrow{\mathrm{r}})=\left\{v(\overrightarrow{\mathrm{r}})-2 \mu \sigma B(\overrightarrow{\mathrm{r}})+u([\vec{n}] ; \overrightarrow{\mathrm{r}})+v_{\mathrm{xc}}^{\sigma, \text { approx }}\left(\left[\bar{n}_{\uparrow}, \bar{n}_{\downarrow}\right] ; \overrightarrow{\mathrm{r}}\right)\right\}-\left\{u\left(\left[\bar{n}_{\alpha \sigma}\right] ; \overrightarrow{\mathrm{r}}\right)+v_{\mathrm{xc}}^{\uparrow, \text { approx }}\left(\left[\bar{n}_{\alpha \sigma}, 0\right] ; \overrightarrow{\mathrm{r}}\right)\right\} .
$$

The self-interaction correction to the potential [the second curly brace in Eq. (38)] appears as a natural consequence of the correction to the energy. For a one-electron system, Eq. (38) correctly reduces to the external potential $v(\overrightarrow{\mathrm{r}})-2 \mu \sigma B(\overrightarrow{\mathrm{r}})$. Equations (32) and (37) form the basis of the SIC approach.

When applied to an electron gas of uniform density $\bar{n}$, Eq. (37) admits plane-wave orbitals as self-consistent solutions, for which the SIC-LSD total energy per electron is exact. Conceivably, however, there might be another set of self-consistent solutions to Eq. (37), with localized orbital densities adding up to a uniform total density, for which the SIC-LSD energy per electron lies below the exact value. Since the orbitals cannot be localized to a region of radius less than $r_{s}=(3 / 4 \pi \bar{n})^{1 / 3}$, any resulting contamination of the total energy from self-interaction correction should be minor if it occurs at all.

The energy eigenvalue $\epsilon_{\alpha \sigma}^{\text {SIC }}$ of the SIC single-particle Eq. (37) is 


$$
\begin{aligned}
\epsilon_{\alpha \sigma}^{\mathrm{SIC}}= & \left\langle\bar{\psi}_{\alpha \sigma}\left|-\frac{1}{2} \nabla^{2}+v(\overrightarrow{\mathrm{r}})-2 \mu \sigma B(\overrightarrow{\mathbf{r}})+u([\bar{n}] ; \overrightarrow{\mathrm{r}})+v_{\mathrm{xc}}^{\sigma, \text { approx }}\left(\left[\bar{n}_{\uparrow}, \bar{n}_{\downarrow}\right] ; \overrightarrow{\mathrm{r}}\right)\right| \bar{\psi}_{\alpha \sigma}\right\rangle \\
& -\left\langle\bar{\psi}_{\alpha \sigma}\left|u\left(\left[\bar{n}_{\alpha \sigma}\right] ; \overrightarrow{\mathbf{r}}\right)+v_{\mathbf{x c}}^{\mathrm{t}, \text { approx }}\left(\left[\bar{n}_{\alpha \sigma}, 0\right], \overrightarrow{\mathbf{r}}\right)\right| \bar{\psi}_{\alpha \sigma}\right\rangle,
\end{aligned}
$$

while the uncorrected energy eigenvalue is

$$
\epsilon_{\alpha \sigma}^{\text {approx }}=\left\langle\psi_{\alpha \sigma}\left|-\frac{1}{2} \nabla^{2}+v(\overrightarrow{\mathrm{r}})-2 \mu \sigma B(\overrightarrow{\mathrm{r}})+u([n] ; \overrightarrow{\mathrm{r}})+v_{\mathrm{xc}}^{\sigma, \text { approx }}\left(\left[n_{f}, n_{\downarrow}\right] ; \overrightarrow{\mathrm{r}}\right)\right| \psi_{\alpha \sigma}\right\rangle .
$$

The first terms in Eqs. (39) and (40) are identical except that in Eq. (39) the SIC orbitals $\bar{\psi}_{\alpha_{\sigma}}(\overrightarrow{\mathrm{r}})$ are used instead of the uncorrected orbitals $\psi_{\alpha \sigma}(\vec{r})$. Hence, SIC has not only a direct effect on the energy eigenvalue [the last term in Eq. (39)], but also an indirect effect (change in energy due to modification of the orbitals). Note that the direct change is made of two contributions having opposite signs: The self-Coulomb correction is negative while the self-exchange-correlation correction is positive. Much of the success of the LSD approximation applied to localized configurations is due to the fact that in many systems the indirect SIC effect is small and the two contributions to the direct term show large cancellation. The SIC eigenvalues $\epsilon_{\alpha \sigma}^{\text {SIC }}$ still have the significance of Eq. (22), and the aufbau principle still applies, as can be seen by repeating the arguments of Sec. II A.

A simple approximate relationship exists between the orbital energies of the LSD and STC-LSD approximations for exchange if one neglects the difference between the orbitals $\bar{\psi}_{\alpha \sigma}$ and $\psi_{\alpha \sigma}$ (i.e., self-consistency effects). We find from Eqs. (39) and (40) that

$$
\begin{aligned}
\epsilon_{\alpha \sigma}^{\mathrm{SIC}} & \approx \epsilon_{\alpha \sigma}^{\mathrm{LSD}}-\left(2 U\left[n_{\alpha \sigma}\right]-0.9305\left(\frac{4}{3}\right) \int d^{3} r n_{\alpha \sigma}^{4 / 3}(\overrightarrow{\mathrm{r}})\right) \\
& \approx \epsilon_{\alpha \sigma}^{\mathrm{LSD}}-0.94 \int d^{3} r n_{\alpha \sigma}^{4 / 3}(\overrightarrow{\mathrm{r}}) .
\end{aligned}
$$

Comparison of Eq. (41) with Eq. (36) shows how the self-interaction error of the LSD total energy gets magnified in the LSD orbital energy.

This SIC formalism can be regarded as a density-functional approximation corrected for self-interaction, or equivalently as the Hartree approximation corrected by an approximation for exchange and correlation between different orbitals. Like the Hartree approximation, it introduces an orbital-dependent effective potential [Eq. (38)] which makes the orbitals slightly nonorthogonal. If desired, the optimum set of orthogonal orbitals could of course be found by introducing off-diagonal Lagrange multipliers into Eq. (37), or by other means. ${ }^{44}$ As discussed in Sec. III B, we have found for atoms that orthogonalization of the orbitals obtained from the one-electron Schrödinger equation has only negligible effect on the total energy.

Clearly, in an orbital-functional scheme such as the present one, exchange could be treated ex- actly via Eq. (7), leaving only correlation to be approximated by

$$
E_{c}^{\mathrm{SIC}}=E_{c}^{\mathrm{approx}}\left[n_{\uparrow}, n_{\downarrow}\right]-\sum_{\alpha \sigma} E_{c}^{\mathrm{approx}}\left[n_{\alpha \sigma}, 0\right] .
$$

However, it is not clear that such an unsymmetrical treatment of exchange and correlation is desirable, since it would reproduce the well known pathologies of the Hartree-Fock eigenvalues for valence electrons in metals. ${ }^{3}$

\section{Exchange-correlation hole}

Gunnarsson and Lundqvist ${ }^{40}$ have argued that the LSD approximation (27) gives reasonable results for realistic systems that are formally outside its domain of validity because it exactly satisfies the sum rule Eq. (26) on the number content of the exchange-correlation hole. The density-fluctuation wave-vector analysis of Langreth and Per$\mathrm{dew}^{21,22}$ supports this conclusion, and also shows (at the risk of oversimplification) that the gradient correction does not give the expected improvement over LSD because it fails to satisfy this sum rule.

The LSD exchange-correlation hole $\mathrm{e}^{40}$ is easily deduced from Eqs. (24) and (27):

$$
\rho^{\mathrm{LSD}}\left(\overrightarrow{\mathrm{r}}, \overrightarrow{\mathrm{r}}^{\prime}\right)=\rho_{h}\left(n_{\mathrm{f}}(\overrightarrow{\mathrm{r}}), n_{\mathrm{t}}(\overrightarrow{\mathrm{r}}) ;\left|\overrightarrow{\mathrm{r}}^{\prime}-\overrightarrow{\mathrm{r}}\right|\right),
$$

where $\rho_{h}\left(n_{\uparrow}, n_{\downarrow} ; R\right)$ is the hole in a homogeneous electron gas with spin densities $n_{\uparrow}$ and $n_{\downarrow}$. Note that the LSD hole around the electron at $\overrightarrow{\mathbf{r}}$ is spherically symmetric, i.e., Eq. (43) depends on the observation point $\overrightarrow{\mathbf{r}}^{\prime}$ only through $\left|\overrightarrow{\mathbf{r}}^{\prime}-\overrightarrow{\mathbf{r}}\right|$ : This spherical symmetry is incorrect, but introduces no error into $E_{\mathrm{xc}}$, since the Coulomb interaction $1 /\left|\vec{r}^{\prime}-\vec{r}\right|$ samples only the spherical average of the hole ${ }^{40}$ :

$E_{\mathrm{xc}}=-\frac{1}{2} \int d^{3} r n(\overrightarrow{\mathrm{r}}) \int_{0}^{\infty} d R 4 \pi R\langle\rho(\overrightarrow{\mathrm{r}}, \overrightarrow{\mathrm{r}}+\overrightarrow{\mathrm{R}})\rangle_{\mathrm{sph}}$.

Since $\rho_{h}$ satisfies the sum rule (26), so obviously does $\rho^{\text {LSD }}$.

The hole in SIC-LSD can also be found by inspection of Eqs. (24), (27), and (32):

$$
\begin{aligned}
\rho^{\operatorname{SIC}}\left(\overrightarrow{\mathrm{r}}, \overrightarrow{\mathbf{r}}^{\prime}\right) & =\rho_{h}\left(n_{\uparrow}(\overrightarrow{\mathrm{r}}), n_{\downarrow}(\overrightarrow{\mathrm{r}}) ;\left|\overrightarrow{\mathbf{r}}^{\prime}-\overrightarrow{\mathbf{r}}\right|\right) \\
& -\sum_{\alpha \sigma} \frac{n_{\alpha \sigma}(\overrightarrow{\mathbf{r}})}{n(\overrightarrow{\mathbf{r}})}\left[n_{\alpha \sigma}\left(\overrightarrow{\mathbf{r}}^{\prime}\right)+\rho_{h}\left(n_{\alpha \sigma}(\overrightarrow{\mathrm{r}}), 0 ;\left|\overrightarrow{\mathbf{r}}^{\prime}-\overrightarrow{\mathbf{r}}\right|\right)\right],
\end{aligned}
$$

from which we find 


$$
\int d^{3} r^{\prime} \rho^{\operatorname{SIC}}\left(\overrightarrow{\mathbf{r}}, \overrightarrow{\mathbf{r}}^{\prime}\right)=-\left[\sum_{\alpha \sigma} f_{\alpha \sigma} n_{\alpha \sigma}(\overrightarrow{\mathrm{r}})\right] / n(\overrightarrow{\mathrm{r}}) .
$$

The right-hand side of Eq. (46) reduces to -1 if the occupation numbers $f_{\alpha \sigma}$ are restricted to the values 0 or 1 , which will be the case under the conditions for which the sum rule (26) was derived; otherwise, the right-hand side of Eq. (46) lies between 0 and -1 . Note that $\rho^{\mathrm{SIC}}\left(\overrightarrow{\mathbf{r}}, \overrightarrow{\mathbf{r}}^{\prime}\right)$ is not spherically symmetric about the point $\vec{r}$, even when the orbital densities $n_{\alpha \sigma}(\overrightarrow{\mathrm{r}})$ have been sphericalized; it depends on $n_{\alpha \sigma}\left(\overrightarrow{\mathbf{r}}^{\prime}\right)$ as well as on $\left|\overrightarrow{\mathbf{r}}^{\prime}-\overrightarrow{\mathbf{r}}\right|$. We will show below that the SIC-LSD exchange hole agrees much better with the exact one then the LSD hole does.

The exchange-correlation hole is not known very accurately, even for the uniform electron gas. However, the exact exchange hole may be found from Eq. (7):

$\rho^{x}\left(\overrightarrow{\mathbf{r}}, \overrightarrow{\mathbf{r}}^{\prime}\right)=-\sum_{\sigma}\left|\sum_{\alpha} f_{\alpha \sigma} \psi_{\alpha \sigma}^{*}(\overrightarrow{\mathrm{r}}) \psi_{\alpha \sigma}\left(\overrightarrow{\mathbf{r}}^{\prime}\right)\right|^{2} / n(\overrightarrow{\mathbf{r}})$.

Note that for orthogonal orbitals we find

$$
\int d^{3} r^{\prime} \rho^{x}\left(\overrightarrow{\mathbf{r}}, \overrightarrow{\mathrm{r}}^{\prime}\right)=-\left[\sum_{\alpha \sigma} f_{\alpha \sigma} n_{\alpha \sigma}(\overrightarrow{\mathrm{r}})\right] / n(\overrightarrow{\mathrm{r}}),
$$

the same as the result (46) for the SIC-LSD scheme. Note further that

$$
\rho^{x}(\overrightarrow{\mathrm{r}}, \overrightarrow{\mathrm{r}})=-\left[n_{\uparrow}^{2}(\overrightarrow{\mathrm{r}})+n_{\uparrow}^{2}(\overrightarrow{\mathrm{r}})\right] / n(\overrightarrow{\mathrm{r}}),
$$

an exact result which is also satisifed by the LSD and SIC-LSD approximations for exchange. From Eq. (47) the exact exchange hole is strictly nonpositive, a condition which is also satisfied by LSD but not by SIC-LSD. (The SIC-LSD hole is positive only at large $R$, as shown in Fig. 1, so this error has little effect on the total energy.)

In order to evaluate the exchange hole in LSD and SIC-LSD, we need to know the exchange hole of the homogeneous electron gas. Using plane waves as orbitals in Eq. (47), we find

$$
\begin{aligned}
\rho_{h}^{x}\left(n_{\uparrow}, n_{\downarrow} ; R\right)=-\frac{1}{2}[ & (1+\zeta) n_{\uparrow} F\left(k_{F}^{\dagger} R\right) \\
& \left.+(1-\zeta) n_{\downarrow} F\left(k_{F}^{\downarrow} R\right)\right],
\end{aligned}
$$

where the relative spin polarization is $\zeta=\left(n_{\uparrow}-n_{\downarrow}\right) /$ $n$, the Fermi wave vector is $k_{F}^{\sigma}=\left(6 \pi^{2} n_{\sigma}\right)^{1 / 3}$, and

$$
F(x)=9\left(\frac{\sin x-x \cos x}{x^{3}}\right)^{2}
$$

The SIC scheme describes exchange exactly in the He atom, since two electrons of opposite spin experience only self-exchange. Our results for the exchange hole in the $\mathrm{Ne}$ atom are shown in Figs. 1 and 2. (Similar figures for the exact and LSD hole have been given by Gunnarsson, Jonson, and Lundqvist. ${ }^{45}$ ) The orbitals employed were the analytic HF orbitals of Bagus. ${ }^{46}$ Figure 1 shows
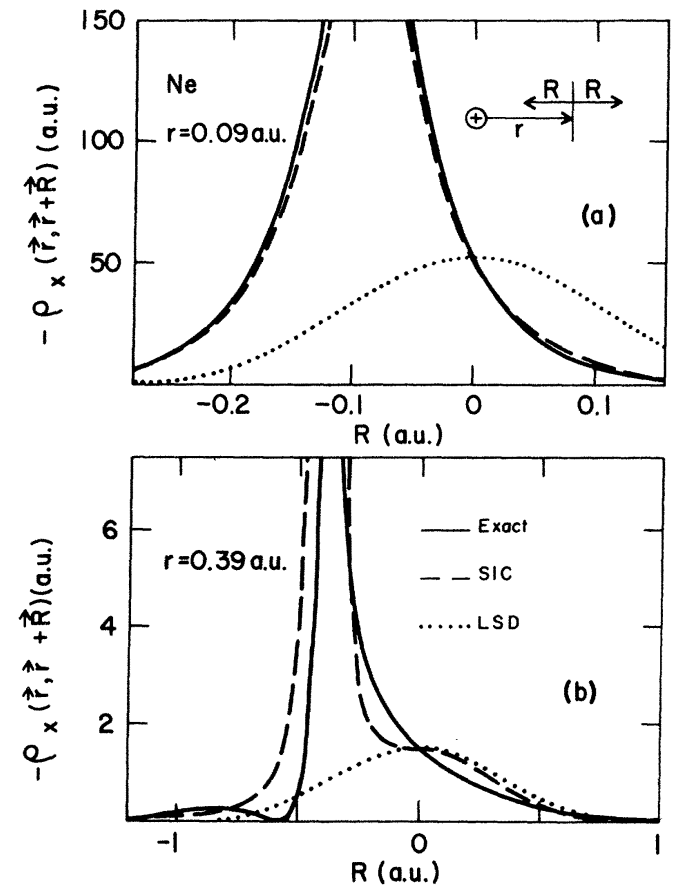

FIG. 1. Exchange hole about an electron located at distance $r$ from the nucleus in the neon atom. The full curves are exact, while the dashed and dotted curves represent the SIC-LSD and LSD approximations, respectively. Parts (a) and (b) are for two different values of $r$. Note that the observation point $\vec{r}^{\prime}=\vec{r}+\vec{R}$ lies on a line with the electron and the nucleus.

that SIC-LSD gives a much more realistic description of the shape of the hole, which peaks up strongly when the observation point $\overrightarrow{\mathbf{r}}^{\prime}$ is near the nucleus, than does LSD. Figure 2 shows that SIC also improves $-R\langle\rho(\overrightarrow{\mathrm{r}}, \overrightarrow{\mathrm{r}}+\overrightarrow{\mathrm{R}})\rangle_{\text {sph }}[$ the spherical average which by Eq. (44) yields the exchange energy] when the electron at $\vec{r}$ is close to the nucleus [Fig. 2(a)], but not necessarily when the electron is further out [Fig. 2(b)]. The improved description of the exchange-correlation hole in the SIC scheme will show up as a substantial improvement in the atomic exchange, correlation, and total energies, presented in Sec. III.

\section{Eigenvalues and removal energies}

Much of the utility of the Hartree-Fock theory lies in Koopmans's theorem, which states that the change in HF total energy due to removal of an electron from an unrelaxed orbital $\alpha \sigma$ is simply related to the $\mathrm{HF}$ eigenvalue:

$$
\left.\Delta E_{\alpha \sigma}^{\mathrm{HF}}\right|_{\text {unrel }}=-\epsilon_{\alpha \sigma}^{\mathrm{HF}} .
$$

Relaxation effects upon removal energies can be calculated in the HF theory by performing two independent self-consistent-field (SCF) calculations 

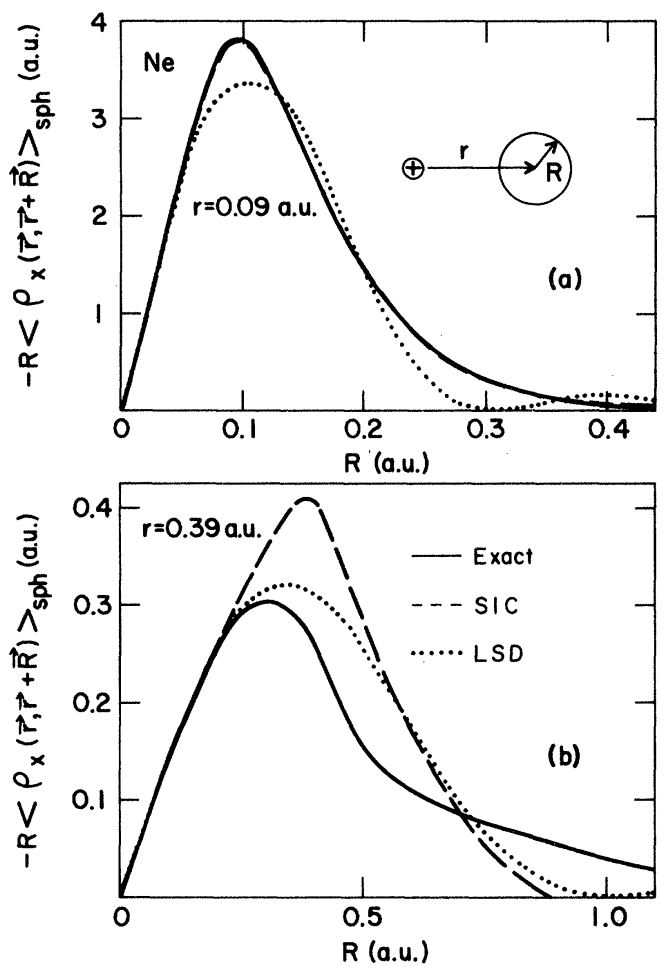

FIG. 2. Spherical average of the neon exchange hole times $R$ for (a) $r=0.09$ a.u. and (b) $r=0.39$ a.u. The full, dashed, and dotted curves are the exact, SIC-LSD and LSD results, respectively. In part (a), the SIC-LSD curve is almost indistinguishable from the exact one.

for the inital and final states (" $\triangle \mathrm{SCF}$ " method); the resulting energy difference is used to define the orbital relaxation energy $\Sigma_{\alpha_{0}}^{\mathrm{HF}}$ :

$$
\left.\left.\Delta E_{\alpha \sigma}^{\mathrm{HF}}\right|_{\text {rel }} \equiv \Delta E_{\alpha \sigma}^{\mathrm{HF}}\right|_{\text {unrel }}+\Sigma_{\alpha \sigma}^{\mathrm{HF}} \text {. }
$$

The relaxation energy $\Sigma_{\alpha \sigma}^{\mathrm{HF}}$ is negative, since relaxation lowers the energy of the final state relative to a non-self-consistent estimate for the ionized system.

The advantage of having Koopmans's theorem is, of course, that fairly accurate removal energies can be found from a single calculation of the eigenvalues for the $\mathrm{N}$-electron system, without also calculating for the $N-1$ electron system as in a $\triangle \mathrm{SCF}$ approach. (If the relaxation of the orbitals is small, of order $\eta$, the relaxation energy is only of order $\eta^{2}$.)

Koopmans's theorem has been a cornerstone of energy-band theory for bulk solids, surfaces, defects, and interfaces, in which much of the interest is directed toward the energy eigenvalues. We will see below that Koopmans's theorem does not hold in the LSD approximation which is frequently used in electronic-structure calculations. The non-Koopmans correction to it, denoted as $\Pi_{\alpha \sigma}$, is positive. Furthermore, in many applications to localized states (deep defects in insulators and semiconductors, surface states, core excitation energies in solids and molecules, etc.), relaxation effects $\Sigma_{\alpha \sigma}$ are significant. We will demonstrate, however, that for atoms the negative of the SIC-LSD energy eigenvalue, $-\epsilon_{\alpha \sigma}^{\text {SIC, }}$ approximately equals the relaxed excitation energy, due to a remarkable near cancellation between the negative relaxation energy $\Sigma_{\alpha \sigma}$ and the positive non-Koopmans correction $\Pi_{\alpha \sigma}$ in SIC. Such an effective cancellation does not appear in the LSD approach. This cancellation allows the formulation of a physically correct one-electron theory

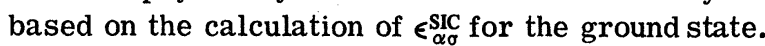

Although many band calculations (pseudopotential or all-electron) now rely on one version of density-functional theory or another, densityfunctional theorists have always viewed the eigenvalues with suspicion. They appear first as purely formal Lagrange multipliers, unredeemed by any Koopmans's theorem. The relationship between removal energy and eigenvalue can be found, however, by integrating Eq. (21):

$$
\left.\Delta E_{\alpha \sigma}\right|_{\text {rel }}=-\int_{0}^{1} d f_{\alpha \sigma} \epsilon_{\alpha \sigma}\left(f_{\alpha \sigma}\right) \text {. }
$$

Here it is the relaxed energy difference or physical removal energy that is obtained, but the eigenvalue must be calculated, using the relaxed orbitals, for occupation numbers $f_{\alpha \sigma}$ between 0 and 1 . Clearly, the use of Eq. (54) would undermine the simplicity of band theory, which seeks a description of excitations in terms of differences between fixed energy levels. Equation (54) was first discovered within $X \alpha$ theory by Slater, ${ }^{5}$ who proposed approximating it via the "transition state"s,47 of half-occupancy:

$$
\left.\Delta E_{\alpha \sigma}\right|_{\text {rel }} \approx-\epsilon_{\alpha \sigma}\left(\frac{1}{2}\right)
$$

Obviously, evaluation of Eq. (54) or even Eq. (55) can be difficult, since the "transition state" may lack the symmetry of the initial state.

Contrary to popular prejudice, Eq. (54) is also true in HF theory once we admit fractional occupation numbers [with the exchange energy defined by Eq. (7)]. An approximation to the integral of Eq. (54), which avoids the need to calculate for fractional occupation numbers, is

$$
\left.\Delta E_{\alpha \sigma}^{\mathrm{HF}}\right|_{\mathrm{re1}} \approx-\frac{1}{2}\left[\epsilon_{\alpha_{\sigma}}^{\mathrm{HF}}(1)+\epsilon_{\alpha_{\sigma}}^{\mathrm{HF}}(0)\right]
$$

Equation (56) was derived in a very different way by Hedin and Johansson, ${ }^{48}$ using the HF "polarization potential".

In order to have an analog of Koopmans's theorem, one would need $\epsilon_{\alpha \sigma}(1)$ (the eigenvalue at full occupancy) in place of $\epsilon_{\alpha_{\sigma}}\left(\frac{1}{2}\right)$ in Eq. (55). However, 
in the $X \alpha$ and LSD approximations, $\epsilon_{\alpha_{\sigma}}$ depends strongly on $f_{\alpha_{\sigma}}$ and no analog of Koopmans's theorem is possible. This result is an artifact of the self-interaction error of the local approximation, and not a general feature of density-functional theory. Even in the hydrogen atom, the LSD eigenvalue varies dramatically as $f_{\alpha \sigma}$ is reduced from 1 to 0 , while the eigenvalue would be independent of $f_{\alpha_{\sigma}}$ for the exact density-functional satisfying Eq. (29). In a self-interaction-free calculation for a system with more than one electron, the eigenvalue $\epsilon_{\alpha_{\sigma}}$ will depend only indirectly on $f_{\alpha_{\sigma}}$, through the reflection back onto orbital $\alpha_{\sigma}$ of its self-consistent effect on the other orbitals, i.e., through physical (rather than spurious) relaxation effects. Physical (interelectron) relaxation effects are easily described: When an electron is removed from an atomic orbital $\alpha \sigma$, i.e., when $f_{\alpha_{\sigma}} \rightarrow 0$, the remaining orbitals are drawn closer to the nucleus, which becomes more effectively screened. This makes $\epsilon_{\alpha_{\sigma}}\left(\frac{1}{2}\right) \geqslant \epsilon_{\alpha_{\sigma}}(1)$, i.e., in a self-interaction-free theory one expects that

$$
\left.\Delta E_{\alpha \sigma}\right|_{\mathrm{rel}} \leqslant-\epsilon_{\alpha \sigma}(1) \text {. }
$$

Since the screening effect from the physical relaxation is often small, the inequality (57) is often close to an equality, yielding a powerful if approximate analog of Koopmans's theorem.

Closed-form expressions for the change in unrelaxed total energy can be found by evaluating energy expressions such as Eq. (15) with fixed orbitals for $f_{\alpha \sigma}=1$ or 0 , and taking the difference. Neglecting correlation terms for simplicity (inclusion of correlation terms poses no difficulty), we find that the LSD and SIC-LSD approximations for exchange yield the simple results:

$$
\begin{aligned}
& \left.\Delta E_{\alpha \sigma}^{\mathrm{LSD}}\right|_{\text {unrel }}=-\epsilon_{\alpha \sigma}^{\mathrm{LSD}}+\Pi_{\alpha \sigma}^{\mathrm{LSD}}, \\
& \left.\Delta E_{\alpha \sigma}^{\mathrm{SIC}}\right|_{\text {unrel }}=-\epsilon_{\alpha \sigma}^{\mathrm{SIC}}+\Pi_{\alpha \sigma}^{\mathrm{SIC}},
\end{aligned}
$$

where the "non-Koopmans" corrections are

$$
\begin{aligned}
& \Pi_{\alpha \sigma}^{\mathrm{LSD}}=U\left[n_{\alpha \sigma}\right]+S_{\alpha \sigma}, \\
& \Pi_{\alpha \sigma}^{\mathrm{SIC}}=-\frac{1}{3} \int d^{3} r n_{\alpha \sigma} \epsilon_{x}\left(n_{\alpha \sigma}, 0\right)+S_{\alpha \sigma},
\end{aligned}
$$

and

$$
\begin{aligned}
S_{\alpha \sigma}=\int d^{3} r & {\left[\left(n_{\sigma}-n_{\alpha \sigma}\right) \epsilon_{x}\left(n_{\sigma}-n_{\alpha \sigma}, 0\right)\right.} \\
& \left.-n_{\sigma} \epsilon_{x}\left(n_{\sigma}, 0\right)+\frac{4}{3} n_{\alpha \sigma} \epsilon_{x}\left(n_{\sigma}, 0\right)\right] .
\end{aligned}
$$

All quantities in Eqs. (58)-(62) are to be evaluated at full occupancy. The analogs of Eqs. (58) and (60) within $X \alpha$ theory were derived by Gopinathan, ${ }^{49}$ and less directly by Slater and Wood. ${ }^{2}$ Note that by Eq. (52), $\Pi_{\alpha \sigma}^{\mathrm{HF}} \equiv 0$.

Two cases are simple enough to be instructive: (i) For a one-electron system, $\Pi_{\alpha \sigma}^{\text {SIC }}$ is clearly zero as it must be, while $\Pi_{\alpha \sigma}^{\mathrm{LSD}} \approx 0.78 \int d^{3} r n_{\alpha \sigma}^{4 / 3}$. (ii) When the orbital density $n_{\alpha \sigma}$ contributes little to the total spin density $n_{\sigma}, S_{\alpha \sigma}$ may be expanded as

$$
S_{\alpha \sigma}=\frac{2}{9} \int d^{3} r n_{\sigma} \epsilon_{x}\left(n_{\sigma}, 0\right)\left(\frac{n_{\alpha \sigma}}{n_{\sigma}}\right)^{2}+O\left(\frac{n_{\alpha \sigma}}{n_{\sigma}}\right)^{3},
$$

or simply neglected. In this case Koopmans's theorem does not hold for localized orbitals in either LSD or SIC-LSD, since the "non-Koopmans" corrections are positive. Using Eq. (34) for estimates, we find that in general

$$
\begin{aligned}
& \Pi_{\alpha \sigma}^{\mathrm{LSD}} \approx 1.09 \int d^{3} r n_{\alpha \sigma}^{4 / 3}+S_{\alpha \sigma}, \\
& \Pi_{\alpha \sigma}^{\mathrm{SIC}} \approx 0.31 \int d^{3} r n_{\alpha \sigma}^{4 / 3}+S_{\alpha \sigma} .
\end{aligned}
$$

As in HF theory, the physical removal energies include a relaxation energy $\Sigma_{\alpha \sigma}$ :

$$
\begin{aligned}
\left.\Delta E_{\alpha \sigma}^{\mathrm{LSD}}\right|_{\text {rel }} & \left.\equiv \Delta E_{\alpha \sigma}^{\mathrm{LSD}}\right|_{\text {unrel }}+\Sigma_{\alpha \sigma}^{\mathrm{LSD}} \\
& =-\epsilon_{\alpha \sigma}^{\mathrm{LSD}}+\Pi_{\alpha \sigma}^{\mathrm{LSD}}+\Sigma_{\alpha \sigma}^{\mathrm{LSD}},
\end{aligned}
$$

and similarly

$$
\left.\Delta E_{\alpha \sigma}^{\mathrm{SIC}}\right|_{\mathrm{rel}}=-\epsilon_{\alpha \sigma}^{\mathrm{SIC}}+\Pi_{\alpha \sigma}^{\mathrm{SIC}}+\Sigma_{\alpha \sigma}^{\mathrm{SIC}} .
$$

While $\Sigma_{\alpha \sigma}$ cannot be calculated analytically, its scaling with simple energy terms in known: Gopinathan $^{49}$ has found by numerical calculations that

$$
\Sigma_{\alpha \sigma}^{\mathrm{LSD}} \approx k_{1} U\left[n_{\alpha \sigma}\right],
$$

with $k_{1}=-0.44$ for atoms. (Better approximations with a shell-dependent $k_{1}$ are possible but will not concern us here.) Equation (68) reduces via Eq. (34) to

$$
\Sigma_{\alpha \sigma}^{\mathrm{LSD}} \approx-0.48 \int d^{3} r n_{\alpha \sigma}^{4 / 3} .
$$

By comparing Eq. (69) with Eqs. (64) and (65), we see that $\Sigma_{\alpha \sigma}^{\mathrm{LSD}}$ can typically cancel less than half of $\Pi_{\alpha \sigma}^{L S D}$, so that the physical removal energy $\left.\Delta E_{\alpha \sigma}^{\mathrm{LSD}}\right|_{\text {rel }}$ is poorly approximated by the orbital energy $-\epsilon_{\alpha \sigma}^{\mathrm{LSD}}$. In contrast, in SIC-LSD there is an effective cancellation between $\Sigma_{\alpha \sigma}$ and $\Pi_{\alpha \sigma}$ leading to a generalized Koopmans scheme in which $-\epsilon_{\alpha \sigma}^{\text {SIC }}$ forms a good approximation to the relaxed ionization energy. Ironically, in the HF theory, which is the only case where Koopmans's theorem holds, no such generalized scheme can exist since there is no "non-Koopmans" term to counteract relaxation effects. As a result, a $\triangle$ SCF approach is often unavoidable in $\mathrm{HF}$. 
From the relations (64)-(69) one can deduce the ordering of terms

$$
\left.\Delta E_{\alpha \sigma}^{\mathrm{LSD}}\right|_{\text {unrel }}>\left.\Delta E_{\alpha \sigma}^{\mathrm{LSD}}\right|_{\text {rel }}>-\epsilon_{\alpha \sigma}^{\mathrm{LSD}} .
$$

If $\Sigma_{\alpha \sigma}^{\text {SIC }}$ is similar to $\Sigma_{\alpha \sigma}^{\text {LSD }}$, it will slightly overcancel $\Pi_{\alpha \sigma}^{\text {SIC }}$, so that

$$
\left.\Delta E_{\alpha \sigma}^{\mathrm{SIC}}\right|_{\text {unrel }}>-\left.\epsilon_{\alpha \sigma}^{\mathrm{SIC}} \gtrless \Delta E_{\alpha \sigma}^{\mathrm{SIC}}\right|_{\text {rel }}
$$

[in agreement with Eq. (57)]. These relationships are illustrated graphically in Fig. 3. Numerical examples are given in Sec. III G. The striking result is that the negative of the SIC orbital energy forms a good approximation to the relaxed removal energy, in contrast to both $L S D$ and $H F$.

Finally, we observe that a simple relationship exists between the unrelaxed energy differences in the LSD and SIC -LSD approximations for exchange when the difference between the LSD and SIC-LSD orbitals is neglected. From Eq. (41) and from Eqs. (58)-(61) we find that

$$
\left.\left.\Delta E_{\alpha \sigma}^{\mathrm{SIC}}\right|_{\text {unrel }} \approx \Delta E_{\alpha \sigma}^{\mathrm{LSD}}\right|_{\text {unrel }}+\delta_{\alpha \sigma}
$$

where $\delta_{\alpha \sigma}$ is the spurious self-interaction of Eqs. (33) and (36). In general, however, higher-order terms (i.e., the self-interaction effects on the orbitals) should not be neglected.

\section{E. Long-range behavior of the potential and density in an atom; excited states}

The LSD one-electron potential for an ion of charge $Q$ has erroneous long-range behavior due to self-interaction, as is evident from inspection of Eqs. (18) and (28): Far from the nucleus the LSD exchange-correlation potential (28) decays exponentially along with the density, leaving only the long-range potentials $v(\overrightarrow{\mathbf{r}})$ and $u([n] ; \overrightarrow{\mathbf{r}})$ which sum to $-Q / r$. However, classical electrostatics

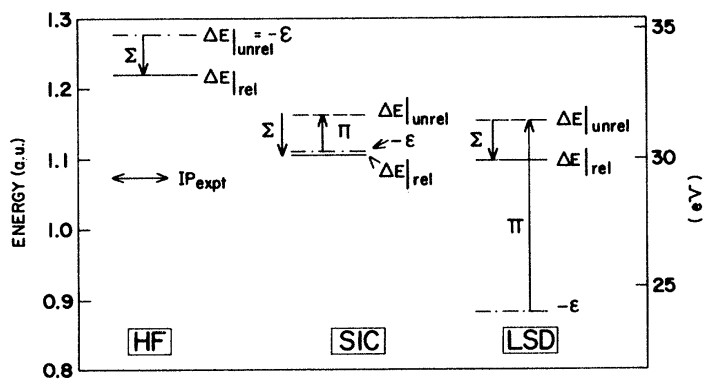

FIG. 3. Relationships between the orbital-energy eigenvalue $\epsilon$, the unrelaxed energy difference $\left.\Delta E\right|_{\text {unrel }}$, and the relaxed energy difference $\left.\Delta E\right|_{\text {rel }}$ upon removal of an electron, in the HF, SIC-LSD, and LSD approximations. In the example shown here, the electron is removed from the $3 s$ orbital in atomic Ar. The relaxation energy $\Sigma$ has been taken from HF calculations. tells us that an electron which ventures out to large $r$ will not "see" its own charge, and so will experience the potential $-(Q+1) / r$; this conclusion is also supported by a detailed analysis of the behavior of the exchange-correlation hole..$^{45}$ The SIC-LSD potential (38), unlike the LSD potential, clearly has the correct long-range behavior. This is necessary for the successful description of negative ions (Sec. IIIE).

After the discussion of Sec. IID, there can be little doubt that the eigenvalues of density-functional theory have physical significance. This is especially true of the eigenvalue $\epsilon_{\max }$ of the leastbound occupied orbital for an $\mathrm{N}$-electron system in its ground state, which by the arguments surrounding Eq. (57) should be almost the negative of the first ionization potential. (Here the initial and final states are ground states of the $N$ and $N-1$ electron systems, and the relaxation effects associated with removal of this outermost electron should be small.) $\epsilon_{\max }$ controls the long-range behavior of the electron density. For example, in an ion of charge $Q$ the one-electron potential tends to $-Z^{*} / r$ as $r \rightarrow \infty$ (where $Z^{*}=Q+1$ in a self-interaction-free theory), and the solution of the oneelectron Schrödinger equation tends to

$$
[n(r)]^{1 / 2}=k r^{\beta} e^{-\alpha r},
$$

where $\beta=Z^{*} / \alpha-1$ and $\alpha=\left(-2 \epsilon_{\max }\right)^{1 / 2}$. In fact if we define $\alpha^{\prime}=(2 I)^{1 / 2}$, where $I$ is the first ionization potential, we find from Eq. (57) that $\alpha^{\prime} \leqslant \alpha$ and hence from Eq. (73)

$$
[n(r)]^{1 / 2} \leqslant k r^{\beta^{\prime}} e^{-\alpha^{\prime} r},
$$

where $\beta^{\prime}=Z^{*} / \alpha^{\prime}-1$, in agreement with the rigorous asymptotic upper bounds of Morrell, Parr, and Levy ${ }^{50}$ and Tal. ${ }^{51}$

Figure 4 illustrates the long-range behavior of the SIC potential $\bar{v}_{\text {eff }}^{\alpha \sigma}(r)$ [Eq. (38)]. We have plotted the difference between the SIC-LSD potential and the LSD potential, both multiplied by $r$, for the $\mathrm{Cu}$ atom in the $3 d^{10} 4 s^{1}$ configuration. At large $r$, this quantity correctly approaches -1 . Note, however, that the self-consistently calculated SIC potentials differ from the LSD potential not only in their asymptotic limit but also at finite $r$ : to set the scale we define the quantities $R_{M}$ and $R_{T}$ which denote the muffin-tin radius of metallic $\mathrm{Cu}$ and the Pauling tetrahedral radius, respectively, while $\langle r\rangle_{n l}$ indicates orbital moments. Clearly the SIC potentials deviate substantially from the LSD potential at distances from the origin which are chemically and physically important.

We close this section with a comment on excited states. The Kohn-Sham equations (17) have solutions that look like states of particle-hole excitation. Although several density-functional theorems 


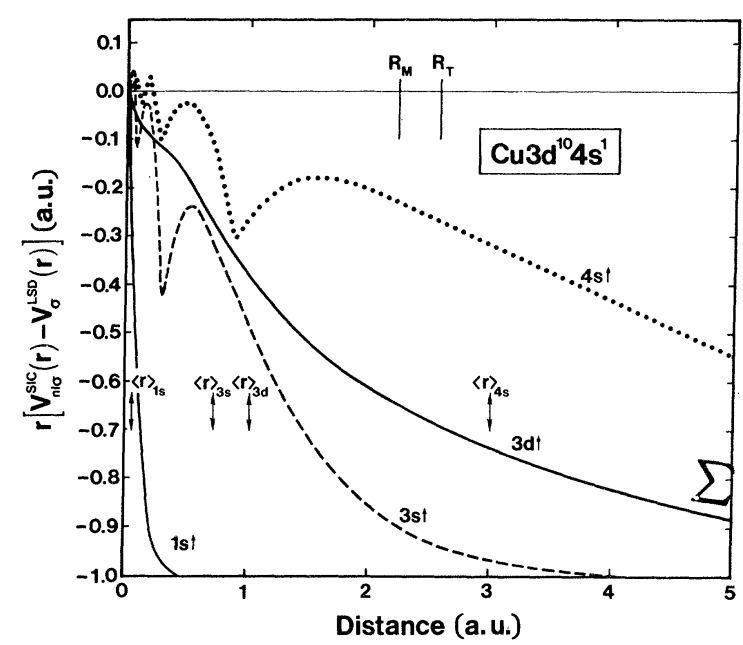

FIG. 4. Orbital-dependent self-interaction correction to the LSD one-electron potential in atomic $\mathrm{Cu}$. The cusps arise from nonanalyticity of $n_{\alpha \sigma}^{1 / 3}(\vec{r})$ at nodes of orbital $\alpha \sigma$. All curves tend to -1 at large $r$.

have been proved about excited states, ${ }^{40,52-54}$ there is usually little a priori justification to interpret the Kohn-Sham solutions as descriptions of physical excitations. Nevertheless, only a purist would discard these Kohn-Sham solutions. While each excitation ought to require a separate self-consistent calculation, in practice one can often just transfer an electron from an orbital occupied in the ground state to a "virtual orbital" (one of the unoccupied eigenfunctions of the ground-state self-consistent one-electron Hamiltonian). In view of our discussion of long-range behavior, the potential used to calculate the virtual orbital ought to be self-interaction free. If we have an orbital-dependent potential as in Sec. II B, the natural way to achieve this is as follows: For excitation of an electron from state $\alpha_{i}$ to state $\alpha_{f}$, compute the virtual orbital $\psi_{\alpha_{f}, \sigma}(\overrightarrow{\mathrm{r}})$ using the potential $v_{\text {eff }}^{\alpha_{i} \sigma}(\overrightarrow{\mathrm{r}})$. The HF analog of this prescription was suggested by Hunt and Goddard. ${ }^{55}$

\section{F. Relationship of SIC to the Hohenberg-Kohn and Kohn-Sham theorems}

In this section we will show that the self-consistent SIC formalism of Sec. II B is protected by the Hohenberg-Kohn theorem of Eq. (11) and so has the same theoretical sanction as other self-consistent density-functional schemes. We will also show that this formalism does not fit into the Kohn-Sham scheme of Eq. (17) (e.g., it has an orbital-dependent potential), although it can be made to fit by minor alterations. (These conclusions apply with equal force to the self-consistent Hartree formalism.)

Since the SIC formalism specifies the total en- ergy as a functional of orbitals and occupation numbers, the SIC energy will be a functional of the density if the orbitals and occupation numbers are. The needed functional relationship is generated by the following rule: Given any pair of trial spin densities $n_{\uparrow}(\overrightarrow{\mathrm{r}})$ and $n_{\downarrow}(\overrightarrow{\mathrm{r}})$, consider all possible occupation numbers $f_{\alpha \sigma}$ in the range $0 \leqslant f_{\alpha \sigma}$ $\leqslant 1$ and normalized orbitals $\psi_{\alpha \sigma}(\overrightarrow{\mathbf{r}})$ satisfying Eq. (13). (The orbitals do not have to be orthogonal.) The correct $f_{\alpha \sigma}$ and $\psi_{\alpha \sigma}(\overrightarrow{\mathrm{r}})$ corresponding to these trial spin densities are the ones which minimize

$$
\sum_{\alpha \sigma}\left(f_{\alpha \sigma}\left\langle\psi_{\alpha \sigma}\left|-\frac{1}{2} \nabla^{2}\right| \psi_{\alpha \sigma}\right\rangle-U\left[n_{\alpha \sigma}\right]-E_{\mathbf{x c}}^{\text {approx }}\left[n_{\alpha \sigma}, 0\right]\right),
$$

with $n_{\alpha \sigma}$ given by Eq. (5). Since the one-electron Schrödinger equation with the orbital-dependent potential (38) results from minimization of the energy over all orbitals, the Hohenberg-Kohn variational principle (11) is valid in the SIC formalism of Sec. II B. (For further discussion, see Appendix A.)

This self-consistent SIC formalism does not fit into the Kohn-Sham scheme because, unlike the Kohn-Sham scheme, it does not define a "kineticenergy" functional of the density which is independent of the choice of $E_{\mathrm{xc}}^{\text {approx }}$. As a result two unusual and possibly unlikable features emerge: (1) The minimizing orbitals that solve the oneelectron Schrödinger equation are slightly but annoyingly nonorthogonal. (2) These orbitals belong to an orbital-dependent potential which can break the symmetry of the system. In a crystal, for example, the core orbitals which minimize the SICLSD total energy are localized, so that the oneelectron potential may not be periodic even when the spin densities are, and Bloch's theorem (with all its computational utility) is lost. (Note, however, that although individual orbitals may not obey this theorem, the total wave function can easily be constructed to have a Bloch periodicity.) While in principle as well as in practice one may, in a perfectly legitimate way, solve problems such as core excitations in molecules or solids using the SIC scheme with nonorthogonal orbitals and broken symmetries, it may also be useful to seek other methods.

These unlikable features could be avoided by incorporating self-interaction corrections within the Kohn-Sham scheme, in which the kinetic-energy density functional has the independent definition of Eq. (14). The simplest scheme which does this is the following ${ }^{34}$ : (1) To each pair of trial spin densities $n_{\sigma}(\overrightarrow{\mathrm{r}})$ there corresponds a pair of model potentials $v_{\text {eff }}^{\sigma}(\overrightarrow{\mathrm{r}})$ which generate these spin densities via Eqs. (13) and (17). $\left[v_{\text {eff }}^{\sigma}(\overrightarrow{\mathrm{r}})\right.$ need not be the self-consistent Kohn-Sham potentials but may be some assumed model potentials.] Solve 
Eq. (17) using these model potentials to find the orbitals $\psi_{\alpha \sigma}(\overrightarrow{\mathrm{r}})$, which are now functionals of the spin density. For small systems such as atoms and ions, the Kohn-Sham orbital densities $n_{\alpha \sigma}(\overrightarrow{\mathbf{r}})$ can be substituted directly into the SIC energy expression. (2) This expression can then be minimized by varying the spin densities; in practice, however, one varies the model potentials $v_{\text {eff }}^{\sigma}(\overrightarrow{\mathrm{r}})$. If the model potentials have sufficient variational flexibility, they will approach a self-consistent solution. This method of potential variation was suggested as a practical scheme for LSD calculations by Rose and Shore. ${ }^{56}$ [The minimization discussed here is constrained to the "Kohn-Sham" space of orbitals belonging to orbital-independent potentials, so the resulting self-interaction-corrected total energy will be greater than the value found by the method of Sec. II B, Eq. (37), which does not impose this constraint.]

The scheme just defined is a Kohn-Sham formalism which is self-interaction free in the sense that it satisfies Eq. (29). Unfortunately, the selfinteraction error reappears in the form of a sizeconsistency problem: The theory will be useful for an isolated atom, but not for a lattice of atoms in the limit of infinite lattice constant, since in the latter case the Kohn-Sham orbitals will be atomiclike Bloch functions and the correction per atom will be zero. As mentioned in Sec. II B, there will in this latter case be a correction to the total energy proportional to the volume of the system to the $\frac{2}{3}$ power, which looks at first like a contribution to the surface energy, but is not; it is simply a shadow of the size-consistency problem. This example, and another discussed in Appendix B, show how subtle the self-interaction problem can be: Satisfaction of Eq. (29) is a necessary condition for solving it but not a sufficient one.

The reason for the size-consistency problem is that the self-interaction correction depends on the choice of orbital representation. For example, the core electrons in a solid could be represented ${ }^{3}$ either by localized Wannier orbitals which carry self-interaction, or by delocalized Bloch orbitals which do not. Stated differently, the self-interaction $\delta_{\alpha \sigma}$ depends on the number of atoms which share the orbital $\psi_{\alpha \sigma}$, as illustrated by the SIC band-structure calculation for solid Ar presented in Sec. II H.

Thus, the key to resolution of the size-consis tency problem lies in unitary transformation of the fully occupied orbitals. Such a transformation leaves invariant a Slater determinant and any oneelectron property that can be evaluated from a Slater determinant, such as the spin density [Eq. (13)], the kinetic energy [Eq. (14)], or the exchange energy $[\mathrm{Eq} .(7)]$. As a result, the Hartree-
Fock and Kohn-Sham total energies are invariant under such a transformation. Since the orbital densities $n_{\alpha \sigma}(\overrightarrow{\mathrm{r}})$ change under the transformation, the SIC total energies are not invariant.

To get a size-consistent self-interaction-corrected Kohn-Sham theory, insert between steps (1) and (2) above another step: (1') Perform a unitary transformation of the Kohn-Sham fully occupied (molecular or Bloch) orbitals to a second set of (more localized) orbitals which will generate the orbital densities to be used in step (2). The right unitary transformation might be the one which minimizes the SIC energy expression. Since the spin densities and the kinetic energy remain invariant, so does each piece of the energy (15) except the self-interaction correction, so this choice amounts to finding the equivalent orbitals that carry maximal self-interaction error. Within SIC-LSD, the transformed orbitals for core electrons in solids will be localized, corresponding roughly to the localized hole that is left behind in x-ray ionization, while loosely bound conduction electrons may have delocalized transformed orbitals.

Finally, although the exact realization of this scheme would be tedious, it is probably unnecessary. LSD already gives reasonable spin densities and one-electron potentials, and it is not hard to imagine an approximate way to incorporate selfinteraction corrections within a self-consistent orbital-independent potential, e.g., by using a weighted average of the SIC orbital-dependent potentials of Eq. (38) [the orbitally-averaged-potential (OAP) method]:

$$
\tilde{v}_{\text {eff }}^{\sigma, 0 A P}(\overrightarrow{\mathrm{r}})=\sum_{\alpha} n_{\alpha \sigma}^{p}(\overrightarrow{\mathrm{r}}) v_{\text {eff }}^{\alpha \sigma}(\overrightarrow{\mathrm{r}}) / \sum_{\alpha} n_{\alpha \sigma}^{p}(\overrightarrow{\mathrm{r}}),
$$

where $p$ is some positive constant. Furthermore, the variational principle suggests that accurate energies could be obtained without optimizing the unitary transformation of the orbitals: It should often suffice to compare the Kohn-Sham orbitals with any unitarily equivalent but more localized orbitals. Such localizing transformations are already familiar in the Edmiston-Ruedenberg ${ }^{57}$ method for molecules and the Wannier transformation for filled bands in solids. ${ }^{3}$

\section{NUMERICAL RESULTS}

\section{A. Ingredients of the calculation}

We have performed numerical calculations using the SIC-LSD formalism of Sec. II B. The parametrized electron-gas correlation energies $\epsilon_{c}$ $\left(n_{\uparrow}, n_{\downarrow}\right)$ are described in Appendix C. They are based on Ceperley's ${ }^{41}$ accurate Monte Carlo calculations for low and metallic densities, which 
we have matched smoothly to the correct highdensity limit. As shown in Appendix C, other parametrized correlation energies commonly used in density-functional calculations are in error at the high densities which are important in atoms.

For atoms and ions, we solve Eq. (37) with the state-dependent potential of Eq. (38) using a direct predictor-corrector numerical integration. At each iteration we have trial spin densities $\left\{n_{\uparrow}(\overrightarrow{\mathbf{r}})\right.$, $\left.n_{\downarrow}(\overrightarrow{\mathbf{r}})\right\}$ as well as trial spin-orbital densities $\left\{n_{n l \sigma}(\overrightarrow{\mathbf{r}})\right\}$. We achieve self-consistency in both sets to a relative accuracy of $10^{-6}$ or a relative accuracy of $10^{-5}$ in the energy (whichever comes first). With the self-consistent orbitals we calculate the total energy and its individual components [e.g., Eqs. (32) and (33)] as well as various moments of $r$. The same calculation is repeated in each case for the LSD approximation.

Following the custom of LSD calculations, we have sphericalized the orbital densities $n_{\alpha \sigma}(\overrightarrow{\mathrm{r}})$ before evaluating the potential and total energy for atoms. This "central-field" approximation is perhaps most severe for the $2 p$ state, which makes a sizable contribution to the total self-interaction correction. However, for a hydrogenic $2 p$ state (with $m_{l}=0$ ) the self-direct Coulomb energy $U\left[n_{2_{p}}\right]$ is only $4 \%$ larger than it would be for the sphericalized orbital density..$^{58}$

The eigenfunctions of the orbital-dependent oneelectron Hamiltonian are very slightly nonorthogonal. Except where otherwise noted, we have performed a Schmidt orthogonalization after each iteration: The $2 s$ orbital is orthogonalized to the $1 s$ then $3 s$ is orthogonalized to $1 s$ and $2 s$, etc.

\section{B. Size of self-interaction terms}

Table II compares the orbital eigenvalues, total energy and separate exchange and correlation energies for the $\mathrm{Kr}$ atom calculated self-consistently in three schemes: LSD, SIC-LSD without orthogonalization, and SIC-LSD with orthogonalization.

The separate orbital contributions to the selfinteraction correction $-\delta_{n l}$ for $\mathrm{Kr}$ are shown in Table III. The sum $-\sum_{l} 2(2 l+1) \delta_{n l}=-185.1 \mathrm{eV}$ is just the difference between the LSD and SIC total energies of Table II, showing that the difference between LSD and SIC-LSD orbitals has little effect on the total energy. Note that even in an atom as large as $\mathrm{Kr}, 44 \%$ of the self-interaction correction to the total energy is carried by the $1 \mathrm{~s}$ orbitals. It is clear from Table II that SIC has a profound effect on all the orbital energies, core as well as valence, and that orthogonalizing the SIC-LSD orbitals is a small effect.

The various self-interaction contributions to the spin-up valence orbital energies $\epsilon_{n l \sigma}^{\text {SIC }}[\mathrm{Eq}$.
TABLE II. Orbital eigenvalues $\epsilon_{n l}$, total energy $E$, and separate exchange $\left(E_{x}\right)$ and correlation $\left(E_{c}\right)$ energies for the $\mathrm{Kr}$ atom, calculated self-consistently in three schemes. Energies in $\mathrm{eV}$. (Throughout the paper the calculations were done in atomic units. We then used the conversion 1 a.u. $=27.21 \mathrm{eV}$.)

\begin{tabular}{lccc}
\hline \hline & \multicolumn{1}{c}{$\begin{array}{c}\text { SIC-LSD } \\
\text { LSD }\end{array}$} & $\begin{array}{c}\text { SIC-LSD } \\
\text { (not orthogonalized) }\end{array}$ & (orthogonalized) \\
\hline$\epsilon_{1 s}$ & -13876.6 & -14127.4 & -14129.5 \\
$\epsilon_{2 s}$ & -1803.6 & -1851.9 & -1852.5 \\
$\epsilon_{2 p}$ & -1633.1 & -1695.0 & -1695.8 \\
$\epsilon_{3 s}$ & -253.46 & -269.46 & -269.54 \\
$\epsilon_{3 p}$ & -192.81 & -209.02 & -209.11 \\
$\epsilon_{3 d}$ & -83.63 & -101.27 & -101.34 \\
$\epsilon_{4 s}$ & -22.30 & -27.76 & -27.76 \\
$\epsilon_{4 p}$ & -9.40 & -13.95 & -13.95 \\
$E$ & -74830.57 & -75015.47 & -75015.47 \\
$E_{x}$ & -2409.06 & -2632.90 & -2631.75 \\
$E_{c}$ & -88.40 & -53.26 & -53.25 \\
\hline \hline
\end{tabular}

are shown diagramatically in Fig. 5 for the $3 d$ elements in the $d^{n-1} s^{1}$ configuration. White areas indicate the self-direct contribution, dashed areas indicate the self-exchange part, while dotted areas denote the self-correlation part. Asterisks mark the SIC eigenvalues $\epsilon_{n l \sigma}^{\text {SIC }}$ while open circles denote the LSD eigenvalues $\epsilon_{n l \sigma}^{\text {LSD }}$. The self-interaction terms are clearly of similar magnitude to the energy eigenvalues themselves. The self-correlation is usually a small correction. For the more localized $3 d$ orbitals, SIC effects are significantly larger than for the more diffuse $4 s$ orbitals. The SIC contributions to the orbital energies follow the same trend as the energy eigenvalues in going from the left- to the right-hand side of the row in the Periodic Table. The large (but incomplete) cancellation between the selfdirect and self-exchange-correlation terms is evident.

TABLE III. Orbital self-interaction corrections $-\delta_{n l}$ [see Eqs. (32) and (33)] to the total energy in the $\mathrm{Kr}$ atom. (SIC-LSD orbitals.) Energies in eV.

\begin{tabular}{ll}
\hline \hline$n l$ & $-\delta_{n l}$ \\
\hline $1 s$ & -40.86 \\
$2 s$ & -5.05 \\
$2 p$ & -9.80 \\
$3 s$ & -1.12 \\
$3 p$ & -1.33 \\
$3 d$ & -2.31 \\
$4 s$ & -0.18 \\
$4 p$ & -0.17 \\
\hline \hline
\end{tabular}




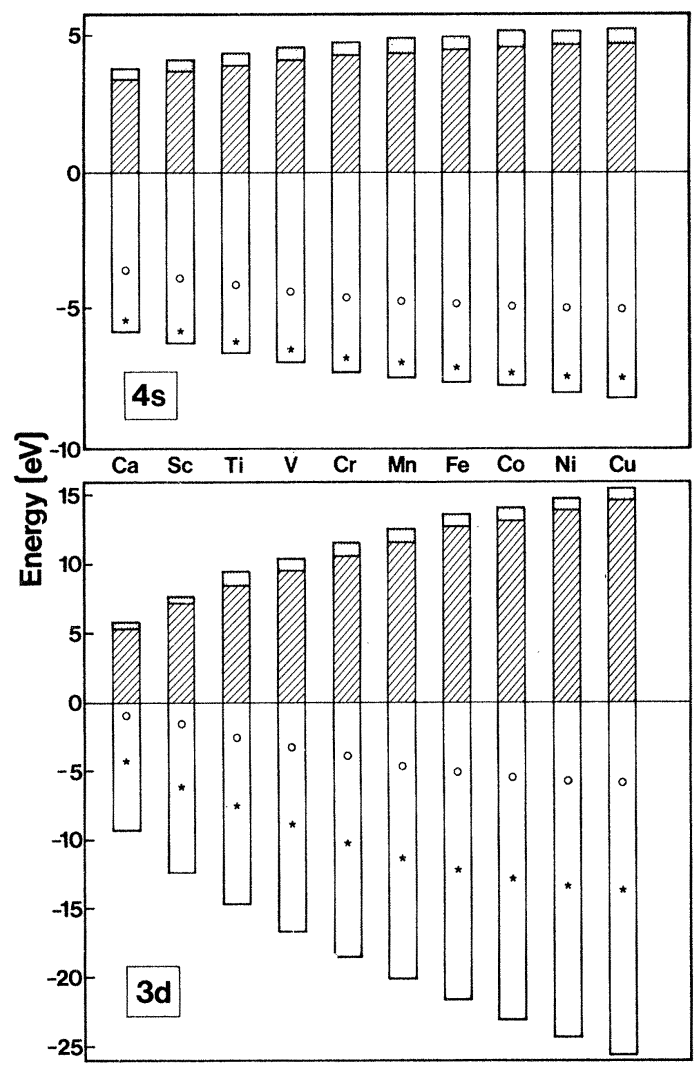

FIG. 5. Breakdown of the orbital energies in the SICLSD formalism for spin-up $4 s$ and $3 d$ orbitals of transition atoms in the $d^{n-1} s \uparrow$ configuration. White area: self-Coulomb; dashed: self-exchange; dotted: selfcorrelation. Open circles: LSD eigenvalues; asterisks: SIC eigenvalues.

\section{Exchange-only calculations compared to Hartree-Fock}

Exchange-only calculations are performed by setting the electron-gas correlation energy $\epsilon_{c}$ $\left(n_{\uparrow}, n_{\downarrow}\right)$ to zero. Table IV shows a comparison of exchange-only orbital eigenvalues, total and kinetic energies, and orbital expectation values $\langle r\rangle$ and $\left\langle r^{2}\right\rangle$ for the Ne atom in LSD, SIC-LSD, and Hartree Fock. ${ }^{59}$ All three approximations satisfy the virial theorem $E=-T$. The LSD orbital values of $\langle r\rangle$ (and also $\left\langle r^{-1}\right\rangle$, not shown) are already remarkably close to Hartree-Fock values, so self-interaction correction provides little improvement in them. The same is true of $\left\langle r^{2}\right\rangle$ except for the loosely bound $2 p$ orbital, which is too diffuse in LSD. On the other hand, the total energy and even more so the orbital eigenvalues have been significantly improved by SIC.

Figure 6 shows the splitting in energy between the spin-up $3 d$ and $4 s$ eigenvalues in the SIC and LSD approximations for the $3 d$ elements. Correla-
TABLE IV. Results of exchange-only calculations for the Ne atom. Here the SIC-LSD orbitals were not orthogonalized. Energies in $\mathrm{eV}$ and distances in a.u.

\begin{tabular}{lrrr}
\hline \hline & & & \\
& LSD & SIC-LSD & $\begin{array}{c}\text { HF } \\
\text { (Ref. 59) }\end{array}$ \\
\hline$\epsilon_{1 s}$ & -822.7 & -888.4 & -891.7 \\
$\epsilon_{2 s}$ & -34.4 & -44.2 & -52.5 \\
$\epsilon_{2 p}$ & -12.1 & -22.0 & -23.1 \\
$E$ & -3469.0 & -3506.2 & -3497.8 \\
$T$ & 3469.0 & 3506.2 & 3497.8 \\
& & & \\
$\langle r\rangle_{1 s}$ & 0.159 & 0.158 & 0.158 \\
$\langle r\rangle_{2 s}$ & 0.906 & 0.908 & 0.892 \\
$\langle r\rangle_{2 p}$ & 0.990 & 0.965 & 0.965 \\
$\left\langle r^{2}\right\rangle_{1 s}$ & 0.034 & 0.034 & 0.033 \\
$\left\langle r^{2}\right\rangle_{2 s}$ & 1.005 & 1.007 & 0.967 \\
$\left\langle r^{2}\right\rangle_{2 p}$ & 1.326 & 1.238 & 1.228 \\
\hline \hline
\end{tabular}

tion effects were not included in this calculation. The results are compared with the HF results of Ref. 59. Note that, for the $d^{n-1} s^{1}$ configuration, LSD predicts that the $3 d$ energies are above the $4 s$ energies for the elements $\mathrm{Sc}$ to $\mathrm{Mn}$, in contrast to the $\mathrm{HF}$ results. The SIC scheme, on the other hand, shows a reasonable agreement with the HF results. Self-interaction corrections may have profound effects on the band structures of the corresponding elemental metals and the energy levels

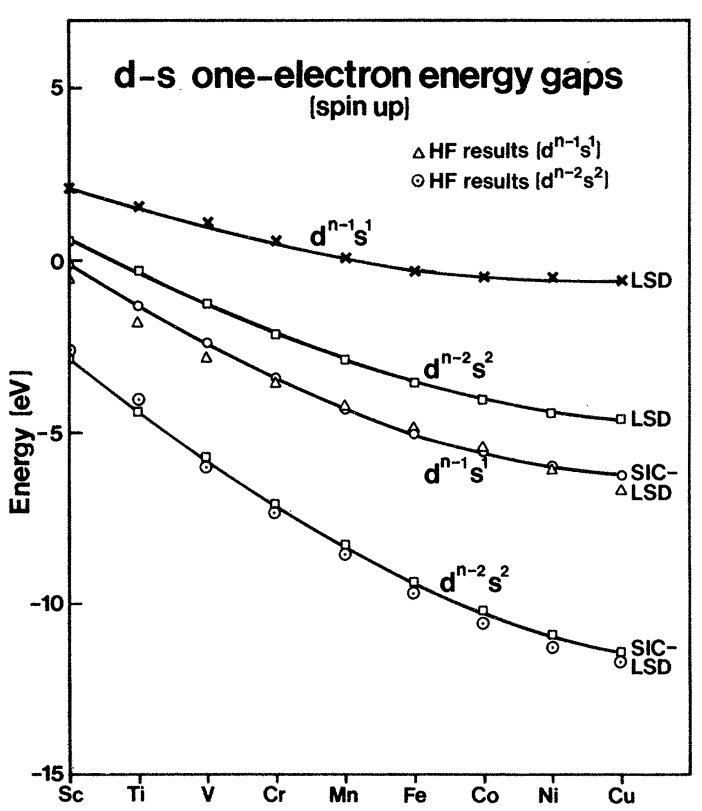

FIG. 6. Difference between $d$ and $s$ orbital energies (spin-up) in LSD, SIC-LSD, and spin-unpolarized HF (Ref. 59) calculations for the $3 d$ elements in the $d^{m-1} s t$ and $d^{n-2} s^{2}$ configurations. 
of transition-element impurities in solids. ${ }^{60}$

Table $\mathrm{V}$ compares the exchange energies of atoms calculated in these three approximations. LSD underestimates the magnitude of the exchange energy by 10-15\%, while SIC-LSD gives values in better agreement with HF. (Note that SIC-LSD for exchange only and HF are the same approximation in the case of atomic $\mathrm{H}$ and $\mathrm{He}$.)

It was once hoped that LSD exchange energies would be improved by including the first corrections from the density-gradient expansion (e.g., the $X \alpha \beta$ method, Ref. 61). For exchange alone (as opposed to exchange and correlation), the a priori gradient correction is negative and so improves the LSD exchange energy, but the improvement is slight for all but the lightest atoms; the coefficient of the gradient term must be artificially magnified by a factor of 5 to get agreement with Hartree-Fock energies. ${ }^{61}$ It might be interesting to reexamine the convergence of the gradient expansion for exchange once each term has been self-interaction corrected.

\section{Total and correlation energies of atoms compared with experiment}

The "experimental" total energy of an $N$-electron atom is the sum of the ionization potentials

$$
\sum_{i=1}^{N} I_{i}
$$

corrected for relativistic and reduced-mass effects. In Table VI we compare experimental values determined this way by Veillard and Clemen$\mathrm{ti}^{62}$ with LSD and SIC-LSD values, which now and hereafter include correlation. The HF values ${ }^{59}$ are of course higher than experiment, and the LSD
TABLE V. Exchange energies of atoms from exchange-only calculations. Here the SIC-LSD orbitals were not orthogonalized. Energies in $\mathrm{eV}$.

\begin{tabular}{lrrr}
\hline \hline Atom & \multicolumn{1}{c}{$E_{x}^{\mathrm{LSD}}$} & $E_{x}^{\text {SIC-LSD }}$ & $\begin{array}{c}E_{x}^{\mathrm{HF}} \\
\text { (Ref. 2) }\end{array}$ \\
\hline $\mathrm{H}$ & -6.9 & -8.5 & -8.5 \\
$\mathrm{He}$ & -23.2 & -27.9 & -27.9 \\
$\mathrm{Ne}$ & -297.6 & -337.8 & -329.5 \\
$\mathrm{Ar}$ & -755.8 & -842.4 & -821.3 \\
$\mathrm{Kr}$ & -2407.5 & -2632.0 & -2561.9 \\
\hline \hline
\end{tabular}

values are higher still, with an error about twice that of HF. The SIC-LSD values, on the other hand, are lower than experiment (because the magnitude of the exchange energy is slightly overestimated, as shown in Table V), with an error magnitude far less than that of $\mathrm{HF}$ for the lighter atoms but comparable to $\mathrm{HF}$ for $\mathrm{Ne}$ and $\mathrm{Ar}$.

The conventional correlation energy $E_{c}^{\text {conv }}$ is defined $^{62}$ as the difference between the experimental total energy and the spin-restricted HF value. The exact spin-density-functional theory defines the correlation energy $E_{c}$ as $E-T-\int v n-U-E_{x}$, with $E_{x}$ given by Eq. (7). The difference between these two definitions arises only from the difference between the spin-restricted HF and KohnSham orbitals, so for spin-unpolarized atoms the variational principle implies the close inequality

$$
E_{c}^{\text {conv }} \geqslant E_{c} \text {. }
$$

Table VII compares the LSD and SIC-LSD correlation energies [the latter evaluated from Eq. (42)] with $E_{c}^{\text {conv }}$. Although the inequality (77) is satisfied, it seems clear that essentially all of the

TABLE VI. Calculated ground-state total energies of atoms compared with experiment (eV). The uncertain Lamb-shift corrections (Ref. 62) were not included in the experimental numbers.

\begin{tabular}{lrrrr}
\hline \hline & $\begin{array}{c}\text { Spin-restricted } \\
E^{\mathrm{HF}} \\
\text { Atom }\end{array}$ & $E^{\text {LSD }}$ & $E^{\text {SIC-LSD }}$ & $\begin{array}{c}\text { Expt. } \\
\text { (Ref. 62) }\end{array}$ \\
\hline $\mathrm{H}$ & -13.6 & -13.0 & -13.6 & -13.6 \\
$\mathrm{He}$ & -77.9 & -77.1 & -79.4 & -79.0 \\
$\mathrm{Li}$ & -202.2 & -199.8 & -204.2 & -203.5 \\
$\mathrm{Be}$ & -396.5 & -393.0 & -399.8 & -399.1 \\
$\mathrm{~B}$ & -667.4 & -662.5 & -672.0 & -670.8 \\
$\mathrm{~N}$ & -1480.2 & -1472.7 & -1488.9 & -1485.3 \\
$\mathrm{~F}$ & -2704.9 & -2696.6 & -2720.7 & -2713.5 \\
$\mathrm{Ne}$ & -3497.8 & -3488.9 & -3517.6 & -3508.1 \\
$\mathrm{Na}$ & -4404.2 & -4392.6 & -4426.1 & -4414.7 \\
$\mathrm{Mg}$ & -5431.5 & -5418.3 & -5456.4 & -5443.2 \\
$\mathrm{Al}$ & -6581.5 & -6566.0 & -6608.8 & -6594.0 \\
$\mathrm{P}$ & -9271.0 & -9251.1 & -9303.7 & -9285.1 \\
$\mathrm{Ar}$ & -14334.7 & -14310.5 & -14378.3 & -14354.6 \\
\hline \hline
\end{tabular}


TABLE VII. Calculated correlation energies of atoms compared with the "conventional" experimental values $E_{c}^{\text {conv }}(\mathrm{eV})$. The uncertain Lamb-shift corrections (Ref. 62 ) were not included in the experimental values.

\begin{tabular}{lccc}
\hline \hline Atom & $E_{c}^{\text {LSD }}$ & $E_{c}^{\text {SIC-LSD }}$ & $\begin{array}{c}E_{c}^{\text {conv }} \\
\text { (Ref. 62) }\end{array}$ \\
\hline $\mathrm{H}$ & -0.6 & -0.0 & -0.0 \\
$\mathrm{He}$ & -3.0 & -1.5 & -1.1 \\
$\mathrm{Be}$ & -6.0 & -3.1 & -2.6 \\
$\mathrm{Ne}$ & -19.9 & -11.4 & -10.4 \\
$\mathrm{Mg}$ & -23.9 & -13.6 & -11.6 \\
$\mathrm{Ar}$ & -38.4 & -22.3 & -19.9 \\
\hline \hline
\end{tabular}

difference between $E_{c}$ and $E_{c}^{\text {conv }}$ arises from errors in our approximations. LSD overestimates the magnitude of the correlation energy by $100-200 \%$ while SIC-LSD overestimates it by only $\sim 20 \%$.

Tong ${ }^{63}$ ascribed the LSD overestimate of the magnitude of the atomic correlation energy to the difference in polarizability between systems with discrete energy levels like atoms and those with continuous energy levels like the electron gas. Since the self-interaction correction arises only for localized orbitals, and localized orbitals imply discrete energy levels, there is perhaps no conflict between his argument and the correctness of the SIC-LSD correlation energies.

Figure 7 shows the LSD and SIC-LSD correlation energies $E_{c}$ for neutral atoms as a function of $N=Z$. Note the change of slope that occurs in the first transition series at $\mathrm{Cr}$, as electrons of antiparallel spin start to be added to the $d$ shell. Ex- cept for such fine details, the LSD and SIC-LSD correlation energies for all atoms and ions may be described by a simple analytic model.$^{64}$

We have established that the SIC-LSD functionals for the total and correlation energies of neutral atoms are rather accurate. Even greater accuracy might be found if the orbital densities were not sphericalized. However, an alternate approach promises to establish a new standard of theoretical accuracy: Levy, Clement, and $\mathrm{Tal}^{65}$ have shown how to get the total energy of an atom of atomic number $Z$ from a knowledge of the first ionization potentials, electron affinities, and $\left\langle r^{-1}\right\rangle$ for neutral atoms with atomic number $\leqslant Z$. Using experimental ionization potentials and affinities, and $\mathrm{HF}$ values of $\left\langle r^{-1}\right\rangle$, they obtained remarkably accurate total and correlation energies. All the inputs needed for this calculation could be computed to excellent accuracy in the SIC-LSD formalism.

\section{E. Binding energies of negative ions}

The negative ions are a sensitive test of any many-electron theory, since a large fraction of their binding energy is due to correlation. In LSD the Kohn-Sham equations do not admit self-consistent negative-ion solutions ${ }^{25}$ : The eigenvalue of the extra electron iterates to a positive value because of the incorrect long-range behavior of the one-electron potential discussed in Sec. II E. (If, however, the extra electron is artificially confined to a region near the atom, the LSD total energy is

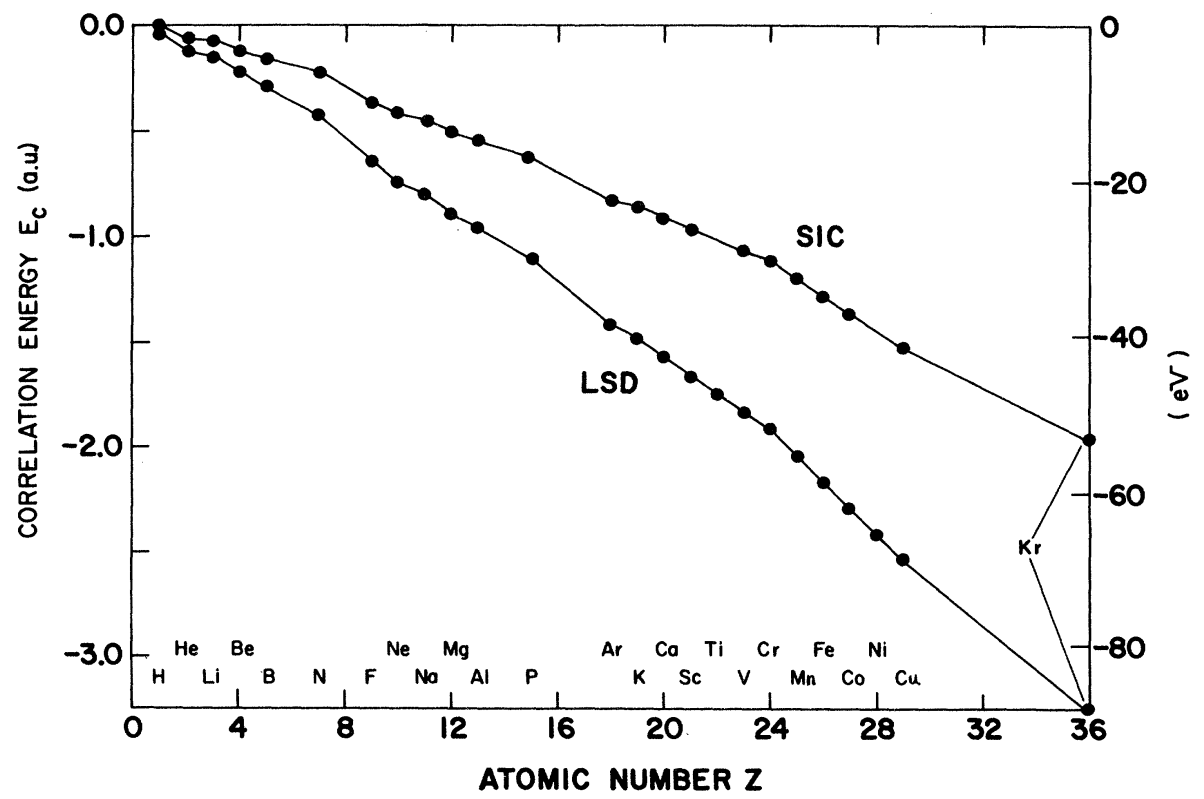

FIG. 7. LSD and SIC-LSD correlation energies of neutral atoms, as a function of atomic number $Z$. 
lower than for the neutral atom, and the difference is a rough estimate of the true binding energy. ${ }^{26}$ ) This kind of misbehavior cannot occur in a selfinteraction-free theory like HF or SIC-LSD: Because of the inequality (57), the eigenvalue of the extra electron will always be negative if binding is allowed by the total energy.

Table VIII shows HF (Refs. 66 and 67) and SICLSD binding energies of negative ions (calculated from total energy differences) in comparision with experiment. ${ }^{68}$ The HF values grossly underestimate the binding, and in some cases predict an instability for the experimentally stable negative ions, while the SIC-LSD values are remarkably close to experiment, giving us additional confidence in the SIC-LSD description of correlation.

\section{F. Density response to the correlation potential}

In Fig. 8 we show the density response to the correlation potential, i.e., the difference between the electron density calculated self-consistently with and without correlation, for the $\mathrm{He}$ atom in LSD and SIC-LSD. The effect of the correlation potential is to build up some extra density near the nucleus by depleting the low-density tail (the region $r>1$ a.u.); the resulting relative change in the density is less than $2 \%$ in the high-density region ( $r<1$ a.u. ), and the relative change may be even smaller in larger atoms where correlation is less important. Just as we found that the SICLSD correlation energy is about half that of LSD in Sec. IIIC, we now find that the SIC-LSD correlation potential is about half that of LSD.

In Fig. 8 we have also plotted the difference between the configuration-interaction (CI) and $\mathrm{HF}$ densities in $\mathrm{He},{ }^{69}$ which can be regarded as the exact density response to the correlation potential. Although the SIC-LSD density is clearly more realistic than the LSD density, both approximations miss the CI tendency to deplete the density in the intermediate region $(r \approx 0.5$ a.u. $)$. Smith, Jagannathan, and Handler ${ }^{70}$ have pointed out how hard it is for approximations based on the uniform electron gas to get this behavior right.

TABLE VIII. Binding energies of negative ions in $\mathrm{eV}$ (or electron affinities of the corresponding neutral atoms).

\begin{tabular}{lccc}
\hline & $\begin{array}{c}\mathrm{HF} \\
\text { (Refs. 66 and 67) }\end{array}$ & SIC-LSD & $\begin{array}{c}\text { Expt. } \\
\text { (Ref. 68) }\end{array}$ \\
\hline $\mathrm{H}^{-}$ & -0.33 & 0.7 & 0.75 \\
$\mathrm{O}^{-}$ & -0.54 & 1.6 & 1.46 \\
$\mathrm{~F}^{-}$ & 1.36 & 3.6 & 3.40 \\
$\mathrm{Cl}^{-}$ & 2.58 & 3.8 & 3.62 \\
\hline \hline
\end{tabular}

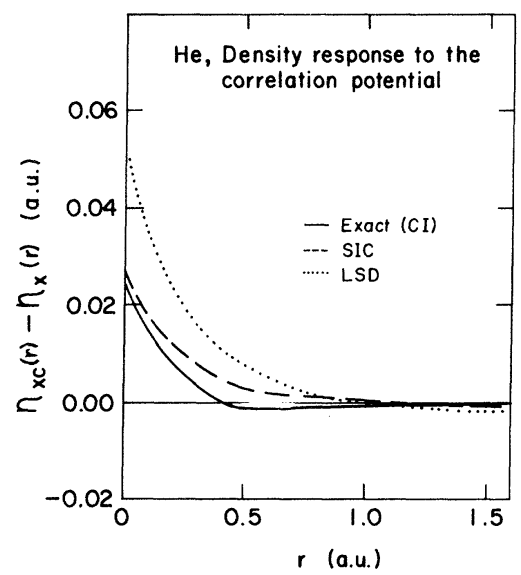

FIG. 8. Density response to the correlation potential in the He atom, in LSD, SIC-LSD, and configurationinteraction (CI) calculations. (The exact or CI curve has been sketched from a figure in Ref. 69.)

\section{G. Eigenvalues and removal energies}

As discussed in Sec. IID, the orbital eigenvalues (multiplied by -1 ) should approximate the relaxed energy changes due to electron removal in SIC-LSD, but not in LSD. This conclusion is supported by Table IX, which compares the eigenvalue of the least-bound occupied orbital to the measured first-ionization potential ${ }^{71}$ in a series of atoms. On the average, the SIC-LSD eigenvalues agree with experiment better than the HF eigenvalues do. They seem to satisfy the inequality (57) almost as an equality, even in He where relaxation effects should be largest. In contrast,

TABLE LX. Comparison of outer-orbital eigenvalues $\epsilon_{\max }$ with measured first ionization potentials $I_{1} \cdot\left(\epsilon_{\max }\right.$ is the least negative eigenvalue of the occupied orbitals.) Energies in $\mathrm{eV}$.

\begin{tabular}{lcccc}
\hline \hline & $\begin{array}{c}-\epsilon_{\max }^{\mathrm{HF}} \\
\text { (Ref. 59) }\end{array}$ & $-\epsilon_{\max }^{\mathrm{LSD}}$ & $-\epsilon_{\max }^{\mathrm{STC}-\mathrm{LSD}}$ & $\begin{array}{c}I_{1} \\
\text { (Ref. 71) }\end{array}$ \\
\hline $\mathrm{H}$ & 13.6 & 7.3 & 13.6 & 13.6 \\
$\mathrm{Li}$ & 5.3 & 3.2 & 5.4 & 5.4 \\
$\mathrm{Na}$ & 5.0 & 3.1 & 5.1 & 5.1 \\
$\mathrm{~K}$ & 4.0 & 2.6 & 4.3 & 4.3 \\
$\mathrm{~N}$ & 15.4 & 8.3 & 14.9 & 14.5 \\
$\mathrm{P}$ & 10.7 & 6.3 & 10.0 & 10.5 \\
$\mathrm{Cr}$ & 6.5 & 4.0 & 6.7 & 6.8 \\
$\mathrm{Mn}$ & 6.7 & 4.6 & 7.1 & 7.4 \\
& & & & \\
$\mathrm{He}$ & 25.0 & 15.5 & 25.8 & 24.6 \\
$\mathrm{Ne}$ & 23.1 & 13.5 & 22.9 & 21.6 \\
$\mathrm{Ar}$ & 16.1 & 10.4 & 15.8 & 15.8 \\
$\mathrm{Kr}$ & 14.3 & 9.4 & 14.0 & 14.0 \\
\hline \hline
\end{tabular}


the LSD eigenvalues only capture about $60 \%$ of the ionization potential.

Qualitatively similar conclusions apply when the ionization process leaves the atom in an excited state. Table $\mathrm{X}$ displays a detailed comparison between ground-state orbital eigenvalues and relaxed energy differences due to hole creation in Ar. First consider the relaxed energy differences $\left.\Delta E\right|_{\text {rel }}$ in comparison with measured removal energies $^{46}$ : The SIC-LSD values show the best overall agreement with experiment, while the $\mathrm{HF}$ values are somewhat in error for the outer orbitals (due to neglect of correlation) and the LSD values are slightly in error for the inner orbitals (due to self-interaction). Note in particular the serious error of the HF approximation for the $3 \mathrm{~s}$ hole in Ar, where $\left.\Delta E^{\mathrm{HF}}\right|_{\text {rel }}$ is $4 \mathrm{eV}$ greater than the experimental removal energy. Bagus ${ }^{46}$ has attributed this error to an anomalous increase in the magnitude of the correlation energy upon formation of the hole. Most of this increase is accounted for within the LSD and SIC-LSD total energy calculations.

Next compare the orbital eigenvalues to the measured removal energies in Table X: The HF eigenvalues overestimate the removal energies from inner orbitals (due to neglect of relaxation and correlation), while the LSD eigenvalues seriously underestimate the removal energies from all orbitals. In contrast the SIC-LSD eigenvalues yield a good estimate of the removal energies. Finally, note that the self-interaction-free theories (HF and SIC-LSD) satisfy the inequality (57).

In Table $\mathrm{X}$ we also show an estimate of the unrelaxed energy difference in SIC-LSD, based on the assumption that the relaxation energy $\Sigma_{\alpha \sigma}^{\text {sIc of }}$ Eq. (67) is the same as $\Sigma_{\alpha \sigma}^{\mathrm{HF}}$ of Eq. (53). Note that in SIC-LSD the negative of the eigenvalue falls between the unrelaxed and relaxed energy differences in agreement with Eq. (71), and lies closer to the relaxed energy difference except for the $1 \mathrm{~s}$ hole.
To what extent can these conclusions carry over into the self-interaction-corrected Kohn-Sham scheme of Sec. II F, in which all orbitals see the same potential? In an attempt to answer this question, we have also calculated the orbital eigenvalues for the Ar atom using the orbitally averaged potential (OAP) of Eq. (75) (with exponent $p=1$ ), and the results are displayed in Table $\mathrm{X}$ where they can be compared to the eigenvalues obtained from the orbital-dependent potential of Eq. (38). There is fair agreement between the two sets of eigenvalues, suggesting that our conclusions can in fact carry over.

\section{H. Band structure of nonmetals}

Turning now to solids, we note that LSD band calculations have had some striking successes in relating the band eigenvalues and related spectral functions to observed excitation. However, in many of the calculations for insulators, if the calculations are pushed to full self-consistency and if additional approximations such as artificial exchange scaling, muffin-tin approximations, empirical charge-transfer factors, or empirical pseudopotentials are avoided, anomalously small optical gaps $E_{g}$ are frequently obtained in LSD [e.g., see Refs. (27)-(31) and Table XI]. Such anomalies normally do not occur in semiempirical pseudopotential calculations, even though LSD is used for screening, ${ }^{72}$ since agreement with the experimental band gaps is usually forced through empirical fitting of the pseudopotential. However, in the first-principles pseudopotential approach ${ }^{73}$ such systematic errors persist. This failure of first-principles band-structure theory with local LSD-type potentials occurs, in fact, for chemically broad classes of nommetals, ranging from van der Waals solids such as $\mathrm{Ne}\left[E_{g}^{\mathrm{LSD}}=11.2 \mathrm{eV},{ }^{29}\right.$ $E_{g}^{\text {expt }}=21.4 \mathrm{eV}$ (Ref. 27)], through covalent solids like $\operatorname{Si}\left(E_{g}^{\mathrm{LSD}}=0.6 \mathrm{eV},{ }^{73(\mathrm{~b})},{ }^{73(\mathrm{c})} E_{g}^{\text {expt }}=1.17 \mathrm{eV}\right)$ to ionic solids like $\mathrm{LiF}\left(E_{g}^{\mathrm{LSD}}=10.5 \mathrm{eV},{ }^{31} E_{g}^{\text {expt }}=14.2\right.$

TABLE X. Comparison of ground-state orbital eigenvalues $\epsilon_{n l}$, relaxed energy differences $\left.\Delta E\right|_{\text {rel }}$, and experimental electron removal energies in atomic Ar. The SIC-LSD column also shows an estimate of the unvelaxed energy differences $\left.\Delta E\right|_{\text {unrel }}$ and eigenvalues calculated with the orbitally averaged potential (OAP). Energies in eV.

\begin{tabular}{|c|c|c|c|c|c|c|c|c|c|}
\hline \multirow{3}{*}{$\begin{array}{l}\text { Hole } \\
\text { state }\end{array}$} & \multirow{2}{*}{\multicolumn{2}{|c|}{ HF (Ref. 46) }} & \multirow{2}{*}{\multicolumn{2}{|c|}{ LSD }} & \multirow[b]{3}{*}{$\left.\Delta E\right|_{\text {urrel }}$} & \multicolumn{2}{|c|}{ SIC-LSD } & \multirow[b]{3}{*}{$-\left.\Delta E\right|_{\mathrm{rel}}$} & \multirow{3}{*}{$\begin{array}{c}\text { Expt. } \\
\text { (Ref. 46) }\end{array}$} \\
\hline & & & & & & $-\epsilon_{n l}$ & & & \\
\hline & $-\epsilon_{n l}$ & $\left.\Delta E\right|_{\mathrm{rel}}$ & $-\epsilon_{n l}$ & $\left.\Delta E\right|_{\mathrm{rel}}$ & & (OAP) & $-\epsilon_{n l}$ & & \\
\hline $1 s$ & 3227.0 & 3195.0 & 3097.0 & 3179.0 & 3227.0 & 3197.0 & 3220.0 & 3195.0 & 3206.0 \\
\hline $2 s$ & 335.0 & 325.0 & 294.0 & 310.0 & 322.0 & 322.0 & 316.0 & 311.0 & \\
\hline $2 p$ & 260.0 & 249.0 & 230.0 & 248.0 & 263.0 & 260.0 & 257.0 & 251.0 & 249.0 \\
\hline $3 s$ & 34.8 & 33.2 & 24.0 & 29.9 & 31.6 & 32.3 & 30.2 & 30.1 & 29.2 \\
\hline $3 p$ & 16.1 & 14.8 & 10.4 & 15.7 & 17.2 & 18.2 & 15.8 & 15.8 & 15.8 \\
\hline
\end{tabular}


eV) and transition-metal oxides like CoO (predicted erroneously by LSD to be metallic ${ }^{74}$ ). While for years such anomalies were often disguised by computational devices which "fixed" the gaps, it has become clearer in the last few years ${ }^{31,73(b), 73(c), 75,76}$ that they reflect a fundamental limitation of the LSD band-structure model. In what follows we will present evidence (see also Refs. 31, 75, and 76 for our earlier corrections to LSD band gaps due to SIC) that these discrepancies arise largely (but not entirely) from self-interaction errors, and that the SIC scheme can form the basis for a new and improved band theory.

It is not difficult to see why self-interaction corrections should significantly alter the results of band theory for atomic crystals like solid Ar (Ref. 77): Although an electron should see an $\mathrm{Ar}^{+}$-like potential in the valence band and an $\mathrm{Ar}^{0}-$ like potential in the conduction band, LSD actually forces an $\mathrm{Ar}^{0}$-like potential for both bands. The difference between the two corresponding orbital energies has both a kinetic component $W$ (included in band theory) and an intra-atomic self-direct component $U$ (neglected in LSD but not in SIC). For localized electronic states one expects $U \gtrless W$.

We show in Table XI, along with the LSD bandstructure error $\Delta E_{g}\left(=E_{g}^{L S D}-E_{g}^{\text {expt }}\right)$ for rare-gas solids, the difference $\Delta_{\text {SIC }}$ between the LSD and SIC-LSD eigenvalues of the outermost atomic orbital forming the valence-band maximum (e.g., $2 p$, $3 p, 4 p$ for $\mathrm{Ne}, \mathrm{Ar}$, and $\mathrm{Kr}$, respectively). The correction $\Delta_{\text {SIC }}$ is expected to be accurate for the valence band when the latter is largely atomiclike and unhybridized. This is indeed the case for raregas solids having narrow $p$-like valence bands which are well separated from the lower $s$-like band. We have calculated $\Delta_{\mathrm{SIC}}=\epsilon_{n p}^{\mathrm{LSD}}-\epsilon_{n p}^{\mathrm{SIC}}$ [Eqs. (39) and (40), where $\epsilon_{\alpha \sigma}^{\text {approx }}$ of Eq. (40) is taken as

TABLE XI. Self-interaction corrections $\Delta_{\text {SIC }}$ to the LSD band-structure error $\Delta E_{g}$ in the band gaps of raregas solids and the core ionization in solid LiF. Energies in $\mathrm{eV}$.

\begin{tabular}{lrrrr}
\hline \hline \multicolumn{1}{c}{ Solid } & $E_{\boldsymbol{g}}^{\text {exp }}$ & \multicolumn{1}{c}{$E_{\boldsymbol{g}}^{\mathrm{LSD}}$} & $\Delta E_{\boldsymbol{g}}$ & $\Delta_{\text {STC }}$ \\
\hline $\mathrm{Ne}$ & $21.4^{\mathrm{a}}$ & $11.2^{\mathrm{c}}$ & -10.2 & 9.9 \\
$\mathrm{Ar}$ & $14.2^{\mathrm{a}}$ & $8.3^{\mathrm{c}}$ & -5.9 & 5.8 \\
$\mathrm{Kr}$ & $11.6^{\mathrm{a}}$ & $6.8^{\mathrm{d}}$ & -4.9 & 4.9 \\
$\mathrm{Xe}$ & $9.3^{\mathrm{a}}$ & & & 4.2 \\
$\mathrm{LiF}(\mathrm{Li} 1 s)$ & $64.4^{\mathrm{b}}$ & $47.2^{\mathrm{e}}$ & -17.2 & 18.0 \\
$\mathrm{LiF}(\mathrm{F} \mathrm{2s})$ & $37.6^{\mathrm{b}}$ & $30.1^{\mathrm{e}}$ & -7.5 & 7.9 \\
\hline \hline
\end{tabular}

${ }^{a}$ Reference 27.

${ }^{\mathrm{b}}$ References 28 and 31.

${ }^{c}$ Reference 29.

${ }^{\mathrm{d}}$ Reference 30.

${ }^{\text {e }}$ Reference 31 . the LSD approximation] using exactly the same local exchange-correlation functionals which were employed in the corresponding band-structure calculations (Refs. 29 and 30). Table XI clearly shows that the self-interaction of this orbital accounts for almost $100 \%$ of the LSD error in the insulating gap of the solid. This is also confirmed through an actual SIC band-structure calculation for solid Ar, described below.

A similar argument applies to ionic solids such as the alkali halides, in which the valence band is constructed predominantly from the anion $p$ orbitals. Interestingly, in the past, the discrepancies between the small LDS band gaps and the larger experimental values in these materials were "remedied" by employing an artifically large exchange parameter $\alpha \geqslant 1 .^{78}$ This device restored an apparent agreement with experiment since the valence states, characterized by fairly localized anion $p$ orbitals, were lowered in energy more than the diffuse conduction-band cationlike $s$ orbitals by the artificial enhancement of the (negative) exchange potential. From our present discussion it is clear, however, that such an empirical approach does not treat the true physical origin of the discrepancy.

It is easy to see why such an approach is not a panacea: One could adjust $\alpha$ to make the self-interaction correction to the total energy zero, by choosing in Eq. (33)

$$
\alpha_{\alpha \sigma}^{(1)}=-U\left[n_{\alpha \sigma}\right] / \frac{3}{2} E_{x}^{\mathrm{LSD}}\left[n_{\alpha \sigma}, 0\right],
$$

or to zero the self-interaction correction to the orbital energy, by choosing in Eq. (39)

$$
\alpha_{\alpha \sigma}^{(2)}=-\frac{\left\langle\psi_{\alpha \sigma}\left|u\left(\left[n_{\alpha \sigma}\right] ; \overrightarrow{\mathrm{r}}\right)\right| \psi_{\alpha \sigma}\right\rangle}{\frac{3}{2}\left\langle\psi_{\alpha \sigma}\left|v_{x}^{4, \mathrm{LSD}}\left(\left[n_{\alpha \sigma}, 0\right] ; \overrightarrow{\mathrm{r}}\right)\right| \psi_{\alpha \sigma}\right\rangle} .
$$

Not only are $\alpha_{\alpha \sigma}^{(1)} \neq \alpha_{\alpha \sigma}^{(2)}$ (so that fixing the band gaps may spoil the total energy), but also these exchange parameters are state dependent (so fixing one gap may spoil another one).

A recent example of this situation is the $\mathrm{CuCl}$ band structure. The experimental band gap (3.4 eV) was obtained in an $X \alpha$ band-structure ${ }^{79}$ calculation using $\alpha=1$, while the correct LSD treatment incorporating $\alpha=\frac{2}{3}$ for exchange (plus local correlation) yielded $E_{g}=2.0 \mathrm{eV} .{ }^{75}$ The reason for this is that the top of the valence band in this material is composed largely of the localized Cu $3 d$ orbitals (with $25 \%$ admixture of $\mathrm{Cl} p$ orbitals) which carry a considerable amount of self-interaction whether $\alpha=1$ or $\frac{2}{3}$. The correction due to this self-interaction term has been estimated ${ }^{75}$ from simple atomic models and found to lead to the experimentally correct band gap only if $\alpha=\frac{2}{3}$ (plus correlation) is used, while for $\alpha=1$ the "corrected" band gap is 
$\sim 4.0 \mathrm{eV}$. It is gratifying that an artificial enhancement of the exchange parameter is no longer needed to obtain the correct band gap in such materials, since the use of $\alpha=1$ spoils the agreement between the calculated ground-state bulk properties (equilibrium lattice parameter, cohesive energy, and bulk modulus) and experiment, while the use of $\alpha=\frac{2}{3}$ (plus correlation) produces the correct results. ${ }^{80}$

It is interesting to contrast this situation with the one encountered in HF band-structure studies. HF calculations (e.g., Ref. 4) predict band gaps for insulators that are too large, often by as much as a factor of 2-5 relative to experiment (due to lack of correlation and the inclusion of spurious self-interaction in the conduction band). On the other hand, LSD band-structure calculations show band gaps which are 20-40\% too small (among other reasons due to spurious self-interaction in the valence band). Both the correlated HF results (e.g., Ref. 4) and the self-interaction-corrected LSD results match the experimental gaps reasonsonably well. It would seem, therefore, that LSD forms a better starting point than $\mathrm{HF}$ to calculate band structures, as the needed corrections to the band structure are far smaller than in HF while the bulk ground-state properties are described in LSD at least as well (and usually better) than in HF.

It is likely that SIC could explain additional discrepancies between LSD calculations and experiment for localized states in extended system. For example, the LSD-calculated positions of the localized surface states in the relaxed GaAs(110) surface $^{20}$ are systematically too high in energy by $0.2-0.3 \mathrm{eV}$ relative to experiment, when the energy of the state is measured in both cases with respect to the Bloch-type valence-band maximum. SIC is expected to lower these states relative to the Bloch states. Similarly, nonempirical LSD band-structure calculations on tetrahedrally bonded heteropolar semiconductors and insulators (e.g., BN, CdS, GaAs) ${ }^{20,76}$ as well as alkali and alkali-earth halides ${ }^{81}$ often indicate a systematically too narrow valence-band width. Inspection of the wave functions involved shows that at the bottom of the band (predominantly of cation $s$-like character) the wave function is more localized than it is at the valence-band maximum (predominantly anion $p$-like character). Self-interaction corrections would then tend to lower the valenceband minimum more than the valence-band maximum, leading to a larger bandwidth.

A somewhat similar situation occurs in the Mott insulators $\mathrm{FeO}, \mathrm{CoO}, \mathrm{MnO}$, and $\mathrm{NiO}$ : While photoemission studies indicate that the upper (transition-element $3 d$-derived) valence band strongly overlaps with the lower (oxygen $2 p$-derived) valence bands, local-density band-structure calculations (e.g., Ref. 74) indicate a large gap (up to 8 $\mathrm{eV})$ between the two groups of bands. One expects then that self-interaction corrections will play a central role in reconciling this result with experiment: The $d$-based valence bands, being far more localized spatially than the $2 p$ band, would receive a larger self-interaction correction (and orbital relaxation correction) and move down in energy relative to the $p$ band, reducing if not closing the spurious LSD gap.

LSD band structures are known to exhibit differences with optical experiments not only at threshold but also in the interband region, a few $\mathrm{eV}$ threshold. Such differences usually show up as $0.1-0.5 \mathrm{eV}$ shifts of the calculated peaks in $\epsilon_{2}(\omega)$ relative to experiment. These differences have encouraged theoretical work (e.g., Refs. 82-84) which emphasizes many-body corrections to the spectra, in particular screened electron-hole and exchange effects. These corrections produce a desired shift in the peaks (e.g., a $0.2-\mathrm{eV}$ shift out of the 0.1-0.5 eV shift needed). However, such approaches completely ignore self-interaction corrections (a fundamental one-body effect) which lead to a far larger shift at threshold and may well result in substantial shifts of $\epsilon_{2}(\omega)$ above threshold due both to a direct SIC modification in the singleparticle energies and to the change in orbitals which enter the screened exchange calculations. For example, the LSD band structure of Si predicts a gap of $0.6 \mathrm{eV}$ [Refs. 73(b) and 73(c)], compared with the observed value of $1.17 \mathrm{eV}$. Manyelectron corrections ${ }^{82}$ amount merely to $a \approx 0.2$ $\mathrm{eV}$ shift in the interband region and practically do not affect the value of the band gap.

We also show in Table XI the error of the LSD band-structure prediction of the energy of core electrons, relative to vacuum, in the ionic insulator LiF; again self-interaction correction of the eigenvalue removes most of the discrepancy. Alternately, but with much greater effort, the correct answer could be obtained within LSD by a $\triangle$ SCF calculation of the relaxed total energy difference in the solid ${ }^{31}$ upon removal of the core electron. The significance of self-interaction terms in narrow-band solids has been emphasized by Zunger and Freeman ${ }^{31}$ who have evaluated them directly in a solid-state $\triangle$ SCF approach.

All band-structure techniques which employ inscribed atomic spheres, and solve a radial atomiclike Schrödinger equation inside such spheres, lend themselves naturally to incorporating a simplified SIC. One has to solve an SIC-LSD singleparticle equation within the sphere (rather than an LSD equation) much as in the atomic case (except 
for the modified Wigner-Seitz boundary conditions at the sphere boundary), while the more diffuse wave-function components outside the sphere can be treated in the standard LSD form. The wave functions are normalized over the entire unit cell. This approach constitutes a generalization of the classical Wigner-Seitz treatment of metallic sodi$\mathrm{um}^{85}$ to include both exchange and correlation in a self-consistent fashion. Such a technique would incorporate the major SIC on the localized states and lead to improved band-structure energies and wave functions.

A self-interaction-corrected self-consistent band-structure calculation has been performed ${ }^{86}$ for the valence $(3 p)$ and conduction bands of solid Ar in the fcc structure. A pseudopotential appropriate to the $3 p$ states, and a mixed basis of Gaussians and plane waves, were employed. The resulting pseudo-orbitals $\psi_{j \overrightarrow{\mathrm{k}}}(\overrightarrow{\mathrm{r}})$ were used to find the pseudodensity by summing over the first Brillouin zone and over the three upper valence bands as

$$
n_{p s}(\overrightarrow{\mathrm{r}})=2 \sum_{\overrightarrow{\mathrm{k}}}^{\mathrm{BZ}} \sum_{j=1}^{3}\left|\psi_{j \overrightarrow{\mathrm{k}}}(\overrightarrow{\mathrm{r}})\right|^{2} \text {. }
$$

If there are $M$ atoms per unit cell (where conventionally $M=1$ ), then the integral of $n_{p s}(\overrightarrow{\mathbf{r}})$ over the unit cell equals $6 \mathrm{M}$. The averaged orbital density $n_{p s}(\overrightarrow{\mathrm{r}}) / 6 M$ was used to calculate $\delta_{\alpha_{\sigma}}[\mathrm{Eq} .(33)]$ as well as the self-interaction-corrected potential $v_{\text {eff }}^{\alpha_{\sigma}}(\overrightarrow{\mathrm{r}})\left[\right.$ [Eq. (38)]. Since $n_{p s}(\overrightarrow{\mathrm{r}}) / 6$ approaches the atomic orbital density $\left|\psi_{3 p}(\overrightarrow{\mathrm{r}})\right|^{2}$ in the limit of infinite lattice constant, the resulting band structure for $M=1$ has the correct atomic limit. Preliminary results indicate that at the observed lattice constant, the calculated band gap is $13.8 \mathrm{eV}$, with the Kohn-Sham exchange and $15.0 \mathrm{eV}$ with the Hedin-Lundqvist exchange-correlation (8.2 eV without SIC) which compares favorably with both the experimental value $(14.2 \mathrm{eV})$ and the atomic estimate of Table XI. The valence-band width increases from the LSD value of $1.3 \mathrm{eV}$ to the SICLSD value of $2.4 \mathrm{eV}$. Note, however, that the success of this approach rests on the intuitive choice $M=1$; if the calculation were repeated with larger unit cells, the orbital density $n_{p s}(\overrightarrow{\mathbf{r}}) / 6 \mathrm{M}$ would carry zero self-interaction in the limit of large $M$. This approach clearly exemplifies the size-consistency problem described in Sec. IIF.

More applications of the SIC band-structure method will have to be attempted in the future (either within the practical schemes described here and in Sec. II F or by other schemes) before an efficient computational method will be clearly identified. One would expect such a method to be particularly useful for band-structure calculations of nonmetals as well as for the calculation of the electronic structure of core excitations and exci- tons in solids, localized surface and interface states, $f$-band materials and valence fluctuations, and deep defect levels, and for the description of metal-nonmetal transitions.

\section{The "Fermi Statistics" problem in Fe and Co}

A peculiar behavior occurs in LSD calculations for atomic $\mathrm{Fe}$ and $\mathrm{Co}$ : If the occupation numbers are restricted to int eger values ( 0 or 1$)$, then the orbital configuration which minimizes the total energy violates the aufbau principle. For example, in Fe the calculated lowest-energy configuration is the observed one $d^{6} s^{2}$, but in this configuration the eigenvalue of the occupied $4 s \downarrow$ orbital lies above the eigenvalue of the four unoccupied $3 d \downarrow$ orbitals. Slater ${ }^{5}$ argued that the minimizing configuration must then have fractional occupation $\left(d^{6.4} s^{1.6}\right.$ in this case $\left.{ }^{12}\right)$.

Fractional occupation is not allowed in the original Kohn-Sham scheme, as Janak ${ }^{37}$ and Harris ${ }^{87}$ have pointed out. From the discussion of Sec. II A, we know that fractional occupation is now permitted in principle. The more practical problem of finding a realistic approximation for the exchange-correlation energy under fractional occupation remains; this problem is not solved by our scheme of self-interaction correction except in certain idealized cases, as discussed in Appendix B.

The SIC-LSD scheme of Sec. II B evades the Fermi statistics problem in the following way: When this scheme is applied to $\mathrm{Fe}$ in the $d^{6} s^{2}$ configuration, the eigenvalue for the occupied $d \downarrow$ orbital lies below that of the occupied $s \downarrow$ orbital, while the eigenvalue of the unoccupied $d \downarrow$ orbitals lies above $s \downarrow$, so the aufbau principle is satisfied. In the self-interaction-corrected Kohn-Sham scheme defined in Sec. II F, all the $d \downarrow$ orbitals will be degenerate and we expect that the Fermi statistics problem may reappear.

\section{J. Cohesive energies of transition metals}

The cohesive energy $E_{\text {con }}$ is the small (1-6 eV) difference of two large numbers: the energy of the free atom and the energy per atom of the solid. Although LSD-calculated total energies of atoms and solids are in error due to self-interaction, their difference yields a reasonable estimate for the cohesive energy in most metals. ${ }^{12}$ In $\mathrm{K}$ for example, where the LSD self-interaction error of the atomic energy is $73 \mathrm{eV}$, the LSD cohesive energy is only $1 \mathrm{eV}$, in good agreement with experiment. The explanation is of course that nearly all the self-interaction error is carried by the ionic core, which is essentially the same for the solid as for the atom, whereas the self-interaction 
error for the $\mathrm{K} 4 s$ orbital is much smaller than the cohesive energy.

The $3 d$ transition metals are a notable exception to this rule. Figure 9 shows a comparison of LSD (Ref. 12) and measured ${ }^{88}$ cohesive energies across the $3 d$ series. Note that LSD gives accurate cohesive energies at both ends of the series, but overestimates them by as much as $70 \%$ near the middle.

As we cross the transition series from $\mathrm{Ca}$ to $\mathrm{Cu}$, the $3 d$ band is progressively filled, and the orbitals contract in response to the increasing nuclear charge. As a result the LSD self-interaction $\delta_{n l}$ of each orbital grows progressively, as shown in Fig. 10. The $3 d$ orbitals are rather localized and each carries a self-interaction (0.2 to $1.2 \mathrm{eV}$ per electron) that is large on the scale of the cohesive energy, but they are not inert core orbitals until we reach $\mathrm{Cu}$; in fact, they hybridize strongly with the $4 s$ orbitals. As we move to the post-transition elements, the $3 d$ orbitals carry even more self-interaction but they are largely chemically inert when the solid is formed from atoms; this leads to an effective cancellation of SIC core terms in the cohesive energy, rendering LSD a good approximation. It seems likely, then, that the $s-d$ valence shell carries a greater LSD self-interaction error in the transition atom than it does in the solid, leading to the LSD overestimation of the cohesive energy.

Let us define the error of the LSD cohesive energy as

$$
\Delta=E_{\mathrm{coh}}^{\mathrm{LSD}}-E_{\mathrm{coh}}^{\mathrm{expt}} .
$$

Following the argument of the preceding paragraph, we equate $\Delta$ to the difference between the LSD self-interaction per atom in the gas and in the

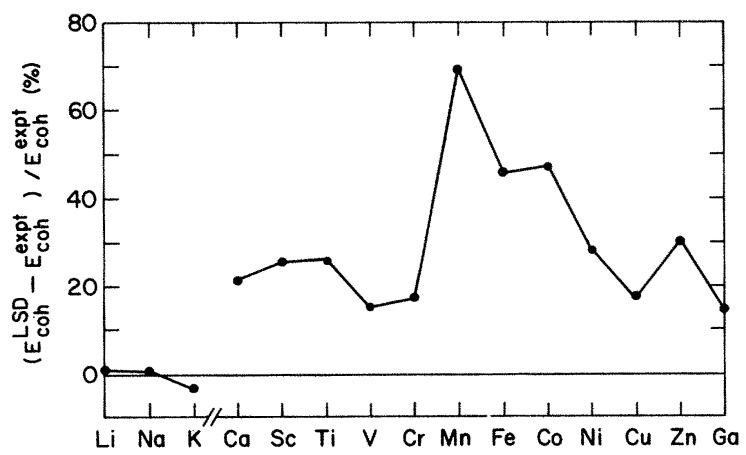

FIG. 9. Percent error in the LSD cohesive energies for some alkali metals and the $3 d$ metals. The LSD and experimental values were taken from Refs. 12 and 88 , respectively. solid:

$$
\begin{aligned}
\Delta & =\sum_{n l} N_{n l} \delta_{n l}^{\text {atom }} \text { (valence) } \\
& -\sum_{j} N_{j} \delta_{j}^{\text {solid }} \text { (valence) }
\end{aligned}
$$

where only valence orbitals contribute [i.e., $\delta_{n l}^{\text {solid }}($ core $) \cong \delta_{n l}^{\text {atom }}($ core $\left.)\right]$. The solid-state selfinteraction $\delta_{j}$ need not be evaluated in the present estimate, but is in principle to be calculated from suitably localized (Wannier-type) functions for the valence bands. We take the $\Delta$ values from Fig. $9, E_{\mathrm{coh}}^{\mathrm{LSD}}$ from Janak et $a l .^{12}$ and $\delta_{n l}^{\text {atom(valence) from }}$ Fig. 10, and calculate $\sum_{j} N_{j} \delta_{j}^{\text {solid }}$ (valence) from Eq. (79). From this we get the ratio between the valence self-interaction in the solid and in the

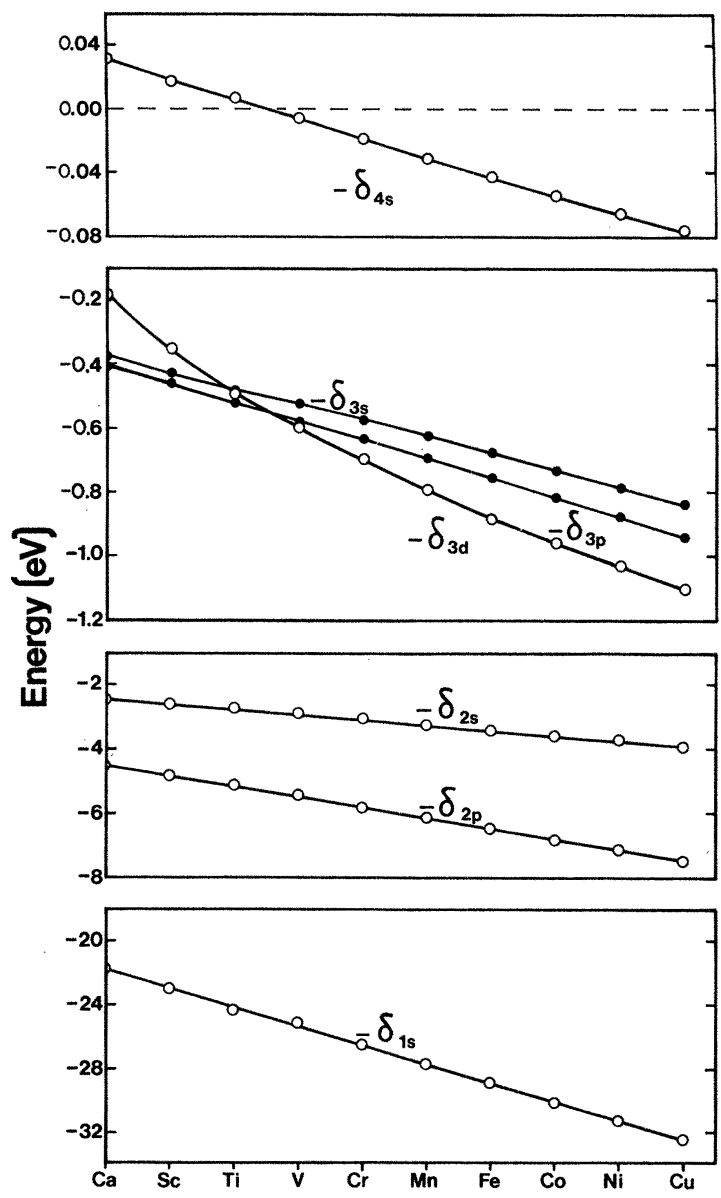

FIG. 10. LSD self-interaction error of the atomic orbitals in the $3 d$ transition series. The self-interaction correction $-\delta_{n l}$ is plotted here for the majority-spin orbitals. This correction is small (and in some cases positive) for the $4 s$ orbitals, but large and negative for the $3 d$ orbitals. 
atom. We find that the valence self-interaction in the solid is negligible for the free-electron metals $\mathrm{Li}$ and $\mathrm{Na}$ [e.g., $\sum_{j} N_{j} \delta_{j}^{\text {solid }}$ (valence) equals 0.00 and $0.01 \mathrm{eV}$ for $\mathrm{Li}$ and $\mathrm{Na}$, whereas the cohesive energies are 1.6 and $1.1 \mathrm{eV}$, respectively], and it varies from $-8 \%$ of the atomic valence self-interaction at the beginning of the $3 d$ series ( $\mathrm{Ti}$ ), through $55 \%$ at the center $(\mathrm{Mn})$ to $86 \%$ at the end of this series (Ni). Such a residual SIC for $d$ electrons in the $3 d$ metals seems physically reasonable and points to the possibility that selfinteraction effects should be considered in transition-metal physics. An a priori calculation of $\delta_{j}$ (valence) in the solid using a suitably localized wave-function representation seems very desirable. Note from Eq. (33) that $\delta_{\alpha \sigma}$ is basically the screened "Hubbard $U$ " (intrasite repulsion term). Our analysis above suggests that the Hubbard repulsion may not be screened out to zero even in elemental transition metals.

\section{K. $s$ - $d$ interconfigurational energies of transition elements}

The interconfigurational energy is the total energy difference between the atomic $d^{n-1} s^{1}$ and $d^{n-2} s^{2}$ orbital configurations. Harris and Jones ${ }^{33}$ have pointed out that LSD predicts the trends of this quantity across the $3 d$ transition series in good agreement with spectroscopic measurements (Fig. 11), although there is an absolute error of about $1 \mathrm{eV}$ in the LSD results which unduly favors the $d$-rich configuration, leading to the wrong predicted ground state in $\mathrm{Ti}, \mathrm{V}$, and $\mathrm{Co}$. The same error seems to lead to an incorrect description of $s-d$ charge transfer in transition-metal dimers. ${ }^{89}$

It seems at first thought that self-interaction correction should worsen the calculated interconfigurational energies, since from Fig. 10 the correction is negligible for a $4 s$ orbital but substantially negative $(-0.2$ to $-1.2 \mathrm{eV})$ for a $3 d$ orbital. Thus the first-order effect of the self-interaction correction is to favor the $d$-rich configuration even more than LSD does. However, a glance at Fig. 11 shows that this expectation is not met, and that the self-consistent SIC-LSD interconfigurational energies are essentially the same as those of LSD.

For estimates of the correction to the total energy or to the one-electron energy eigenvalues, the self-interaction correction can be treated as a first-order perturbation on the LSD solution (i.e., approximating SIC orbitals by LSD orbitals), but this approach fails for the interconfigurational energy. What happens is that the first-order effect lowers the energy of $d^{n-1} s^{1}$ relative to $d^{n-2} s^{2}$, but the higher-order effects lower the energy of the more polarizable $d^{n-2} s^{2}$ configuration relative to $d^{n-1} s^{1}$, and the se effects essentially cancel one

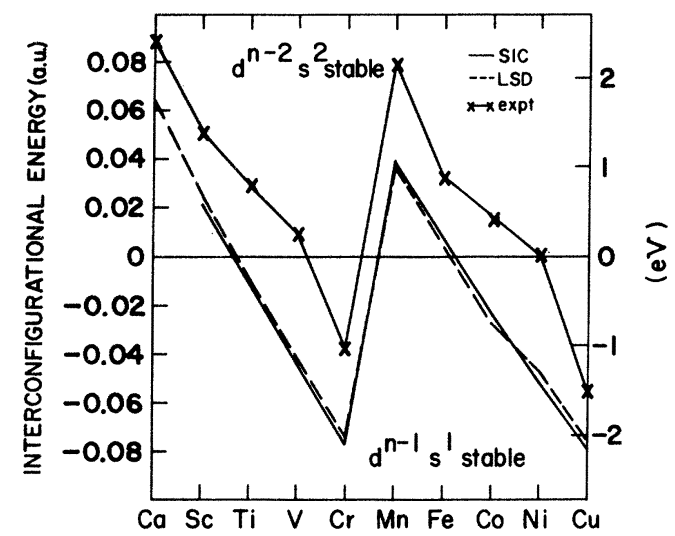

FIG. 11. $s-d$ interconfigurational energies of transition-metal atoms (calculated as total energy differences) compared with experiment (crosses, from Ref. 33). The break in the curve at $\mathrm{Cr}$ occurs because of the Hund's-rule special stability of the $d^{5}$ configuration. The LSD values shown here are about $0.2 \mathrm{eV}$ closer to experiment than those of Ref. 33, due to our use of the accurate Ceperley correlation.

another.

Self-interaction correction neither improves nor worsens the LSD interconfigurational energies. These LSD values are in fact remarkably "persistent": The same values are found in LSD calculations whether the density is sphericalized or not, ${ }^{90}$ and the same values are also obtained from the "weighted density" approximation of Eq. (83) below. ${ }^{45,91}$

\section{Spin splitting in $\mathrm{Fe}, \mathrm{Co}$, and $\mathrm{Ni}$}

The LSD approximation has been successful in predicting the spin susceptibilities of the paramagnetic metals, ${ }^{12,14}$ which are strongly enhanced by exchange and correlation. LSD also correctly predicts that the ground states of $\mathrm{Fe}, \mathrm{Co}$, and $\mathrm{Ni}$ are ferromagnetic, with calculated magnetic moments in good agreement with experiment. ${ }^{12}$ Except for the weak ferromagnet $\mathrm{Ni}$, the LSD band structures of the ferromagnetic metals are in reasonable agreement with the results of angleresolved photoelectron spectroscopy. ${ }^{32}$ For example, the ratio of theoretical to experimental occupied $d$-band width is about $1.1,1.2$, and 1.45 for $\mathrm{Fe}, \mathrm{Co}$, and $\mathrm{Ni}$, respectively, while the ratio of theoretical to experimental spin splitting is about 1.0, 1.2, and 2.2 for the same metals. ${ }^{32}$

The LSD overestimate of the spin splitting in $\mathrm{Ni}$ by a factor of two led us to wonder if self-interaction correction would change the LSD spin splitting. The analog in our atomic calculations of the solid-state band-structure spin splitting is $\epsilon_{3 d} \downarrow$ $-\epsilon_{3 d}$, the difference in eigenvalues of the minority 
$(\downarrow)$ - and majority ( $\uparrow)$-spin $3 d$ electrons. Figure 12 shows this quantity as a function of atomic configuration in LSD and SIC-LSD; the measured solid-state spin splittings are also shown for comparison.

Note that self-interaction correction increases the spin splitting, although the increase for $\mathrm{Ni}$ is only marginal. The reason is very simple: The majority spins see a more attractive exchangecorrelation potential than the minority spins do, and so have more localized orbitals. The selfinteraction correction lowers the eigenvalues of all occupied $d$ orbitals, but the eigenvalues of the more localized majority-spin orbitals get lowered more, leading to an increase in the spin splitting. [This argument, due to L. Kleinman and K. Mednick (private communication), is based on treating the self-interaction correction to the one-electron potential as a first-order perturbation on the LSD eigenvalue, a treatment which gives numerical results in rather good agreement with our selfconsistent ones. However, the success of this approach should not be interpreted to mean that the self-interaction correction has no effect on the orbitals of transition-metal atoms; in fact, at selfconsistency it contracts the $3 d$ orbitals and expands the $4 s$ orbitals non-negligibly.]

These atomic calculations suggest that selfinteraction correction could slightly worsen the calculated spin splittings in solid $\mathrm{Fe}, \mathrm{Co}$, and $\mathrm{Ni}$. However, as we observed in our discussion of the cohesive energies (Sec. IIIJ), self-interaction cor-

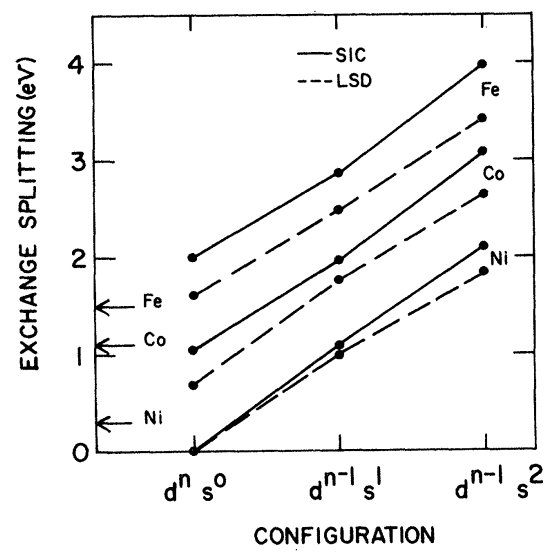

FIG. 12. Spin splitting in atomic $\mathrm{Fe}, \mathrm{Co}$, and $\mathrm{Ni}$ as a function of atomic configuration. The difference between the energy eigenvalues of minority - and majorityspin occupied $3 d$ orbitals is plotted here. (The LSD values for $\mathrm{Fe}$ and $\mathrm{Co}$ in the $d^{n} s^{0}$ configuration are uncertain due to convergence difficulties.) The observed (Ref. 32) spin splittings in the solid, indicated by horizontal arrows, are shown for comparison. rections are not the same in the solid transition metals as in the corresponding free atoms, so these atomic calculations may not be very predictive of solid-state effects.

\section{BRIEF REVIEW OF RELATED WORK}

When we separately began to work on self-interaction corrections, we knew about the work of Lindgren. ${ }^{92}$ Since then we have learned of other related studies. Here we attempt a brief critical review of the papers we have found, in relation to our own work. Except where otherwise indicated, this related work was directed toward removing the self-interaction error of the one-electron potential in the local-density approximation, in contrast to our own work which gives a general scheme for self-interaction correction of any spin-density-functional approximation for the total energy as well as the potential.

Latter ${ }^{93}$ observed that the Slater $n^{1 / 3}(\overrightarrow{\mathrm{r}})$ exchange potential approaches zero exponentially far from the center of a neutral atom, while the HartreeFock exchange potential goes to $-1 / r$, and proposed switching over from the former to the latter form at the value of $r$ where they cross, a scheme followed by Herman and Skillman ${ }^{94}$ in their atomicstructure calculations. From the present work, however, it is clear that self-interaction effects modify the potential even before it reaches its asymptotic $-1 / r$ value (see the difference in orbital-dependent potentials in Fig. 4 for small $r$ ). Cowan ${ }^{95}$. showed that Latter's procedure can introduce errors into calculated ionization energies, and proposed a scheme in which the self-Coulomb potential $u\left(\left[n_{\alpha \sigma}\right] ; \overrightarrow{\mathrm{r}}\right)$ was subtracted from the oneelectron potential seen by orbital $\alpha \sigma$, while the interelectronic exchange potential was parametrized in a complicated way to give a best fit to the Hartree-Fock orbital energies. This scheme gave the correct long-range behavior of the potential, and the orbitals gave a good total energy when inserted in the Hartree-Fock energy functional (not the density-functional expression).

Liberman ${ }^{96}$ proposed partitioning the density of an atom into a sphere containing $N-1$ electrons and the rest of space containing one electron. The total energy then involved local exchange only within the sphere and electrostatic interaction between the two regions. This scheme corrected the self-interaction error for the outermost electron, so that a $\triangle$ SCF calculation gave binding energies of negative ions as good as the Hartree-Fock values or better, but of course it left the large self-interaction error of the inner-shell electrons uncorrected.

Slater and Wood $^{2}$ pointed out that a large fraction of the total exchange energy in atoms is just self- 
exchange. However, they rejected any attempt to treat self-exchange exactly because, unlike the total exchange energy, it is not invariant under unitary transformation of the occupied orbitals. We discussed this fact, and how it may be exploited, in Sec. II F.

Lindgren's ${ }^{92,97}$ work is perhaps closest to our own. Lindgren and Rosén ${ }^{97}$ calculated the interelectronic exchange energy of a uniform electron gas confined to a finite volume by periodic boundary conditions, and found a correction to the local exchange energy which becomes

$$
-\sum_{\alpha \sigma} \int d^{3} r n_{\alpha \sigma}(\overrightarrow{\mathrm{r}}) \epsilon_{x}\left(n_{\alpha \sigma}(\overrightarrow{\mathrm{r}}), 0\right)
$$

for small electron numbers $N$, and never deviates much from this for large $N$. Using Eq. (77) and the exact self-Coulomb correction $-\sum_{\alpha \sigma} U\left[n_{\alpha \sigma}\right]$ as corrections to the local exchange energy, they variationally derived an orbital-dependent potential which is the same as the one we would find from Eq. (38) within the SIC scheme for local exchange only:

$$
\begin{aligned}
v_{x}^{\alpha \sigma}(\overrightarrow{\mathbf{r}})= & \mu_{x}^{\sigma}\left(n_{\mathfrak{t}}(\overrightarrow{\mathbf{r}}), n_{\downarrow}(\overrightarrow{\mathbf{r}})\right) \\
& -u\left(\left[n_{\alpha \sigma}\right] ; \overrightarrow{\mathbf{r}}\right)-\mu_{x}^{\dagger}\left(n_{\alpha \sigma}(\overrightarrow{\mathbf{r}}), 0\right) .
\end{aligned}
$$

In atomic calculations, this scheme gave eigenvalues $\epsilon_{\alpha \sigma}$ much closer to Hartree-Fock than the local exchange eigenvalues. Curiously, the total energies obtained directly from the self-interaction-corrected density functional for the energy were never reported. Instead, the Schrödinger equation with the exchange potential of Eq. (81) was used merely as a wave-function generator for HF calculations. In separate work, Lindgren and Schwarz ${ }^{98}$ showed that the dominance of the selfexchange over interelectronic exchange in small atoms was the reason for the decrease in the optimum $\alpha$ value of Slater's $X \alpha$ theory from $\sim 0.8$ for small atoms to $\sim \frac{2}{3}$, the proper LSD value for exchange, in large atoms.

Szasz, Berrios-Pagan, and McGinn ${ }^{99}$ proposed an approximation for the atomic energy as an explicit functional of the density, including a simple self-interaction correction in the spirit of the Fermi-Amaldi correction. ${ }^{1}$

Schrieffer ${ }^{100}$ briefly discussed the self-interaction error of $X \alpha$ and LSD total energies, and gave an argument qualitatively similar to the one of Eq. (41) to show how this error gets magnified in the orbital eigenvalues $\epsilon_{\alpha \sigma}$.

Talman and Shadwick ${ }^{101}$ showed how to construct an orbital-independent, spatially local exchange potential $v_{x}(\overrightarrow{\mathrm{r}})$ which minimizes the Hartree-Fock total energy. This potential naturally turns out to be self-interaction free, and can be regarded as essentially the exact Kohn-Sham potential for exchange in atoms. In a comparative study of various spatially local exchange potentials $v_{x}(\overrightarrow{\mathrm{r}})$, Talman, Ganas, and Green ${ }^{102}$ found that those which minimized the Hartree-Fock energy either exactly or in a restricted variational sense gave orbital energies $\epsilon_{\alpha \sigma}$ in better agreement with measured removal energies and excitation energies than the Hartree-Fock eigenvalues.

Brandow ${ }^{103}$ pointed out that a local-exchange $X \alpha$ band-structure model fails for Mott insulators such as $\mathrm{CoO}, \mathrm{MnO}$, and $\mathrm{NiO}$ essentially due to the failure of the theory to subtract the self-interaction terms from the potential. He then proposed to construct a $d$-band Hubbard Hamiltonian based on a linear combination of atomic orbitals fit to $X \alpha$ band structure with the self-interaction terms properly subtracted. This model Hamiltonian correctly describes the energy levels and magnetic properties of these systems when empirical values for the self-Coulomb terms are used.

Gopinathan ${ }^{49}$ devised a scheme in which the self-Coulomb correction was treated exactly, as in Lindgren's work, while the interelectronic exchange was approximated as

$$
\begin{aligned}
E_{x}^{i . e .}=\sum_{\sigma} \frac{-9 C}{2} \alpha_{\sigma} \sum_{\alpha} \int & d^{3} r n_{\alpha \sigma}(\overrightarrow{\mathbf{r}}) n_{\sigma}^{-2 / 3}(\overrightarrow{\mathbf{r}}) \\
& \times\left[n_{\sigma}(\overrightarrow{\mathrm{r}})-n_{\alpha \sigma}(\overrightarrow{\mathbf{r}})\right],
\end{aligned}
$$

which is roughly in the form of an interaction between the orbital density $n_{\alpha \sigma}(\vec{r})$ and the remaining spin density $\left[n_{\sigma}(\overrightarrow{\mathrm{r}})-n_{\alpha \sigma}(\overrightarrow{\mathrm{r}})\right]$. The variationally derived potential had the correct long-range behavior, and the eigenvalues and hyperfine contact densities were close to Hartree-Fock values. Again the self-interaction-corrected density functional for the energy served only as an intermediate step in the derivation of the potential and was not itself evaluated.

A Kohn-Sham density functional for the energy which is self-interaction-free [to the extent of satisfying Eq. (29) for fully occupied orbitals] has been proposed by Gunnarsson, Jonson, and Lundqvist, ${ }^{45}$ and independently by Alonso and Girifalco. ${ }^{104}$ This is the "weighted density" approximation, in which the exchange-correlation hole (Secs. II A and II C) is approximated as

$$
\rho\left(\overrightarrow{\mathbf{r}}, \overrightarrow{\mathbf{r}}^{\prime}\right)=n\left(\overrightarrow{\mathbf{r}}^{\prime}\right) \frac{\rho_{h}\left(\tilde{n}(\overrightarrow{\mathbf{r}}),\left|\overrightarrow{\mathbf{r}}^{\prime}-\overrightarrow{\mathbf{r}}\right|\right)}{\tilde{n}(\overrightarrow{\mathbf{r}})},
$$

where $\rho_{h}\left(\tilde{n},\left|\overrightarrow{\mathbf{r}}^{\prime}-\overrightarrow{\mathbf{r}}\right|\right)$ is the hole in a homogeneous electron gas of density $\tilde{n}$. The weighted density $\tilde{n}$ at each $\overrightarrow{\mathrm{r}}$ is determined to satisfy the sum rule (26). This approximation obviously becomes exact in the limit of slow spatial variation of the density, like LSD. Applied to a one-electron system, the sum rule (26) can only be satisfied for 
$\tilde{n}=0$ (the Wigner crystal limit) and then $\rho\left(\overrightarrow{\mathbf{r}}, \overrightarrow{\mathbf{r}}^{\prime}\right)$ $=-n\left(\overrightarrow{\mathbf{r}}^{\prime}\right)$, which is exact; ${ }^{45}$ thus our SIC scheme gives no correction to the weighted density approximation. The weighted density approximation has been tested in non-self-consistent calculations for atoms. It reduces the $\sim 10 \%$ error of the LSD exchange energy to $\sim 4 \%,{ }^{45}$ and greatly improves the exchange energy density. ${ }^{104}$ The error in the integrated exchange energy can be reduced further by a shell-partitioning scheme, ${ }^{45}$ at the cost that the approximation ceases to be a density functional. Unfortunately, the correlation energy is overestimated by about a factor of 2 , as in LSD. ${ }^{45}$ Also, as in LSD, the orbital eigenvalue for the negative-ion $\mathrm{H}^{-}$turns out to be incorrectly positive,${ }^{45}$ while the ionization potentials of atoms (calculated from relaxed energy differences) are somewhat worse than in LSD. ${ }^{91}$ Thus this scheme, which in comparison with SIC-LSD is rather more sophisticated, usually gives less satisfactory numerical results.

Bryant and Mahan ${ }^{105}$ used a form of self-interaction correction in the potential to calculate soft $\mathrm{x}$-ray absorption and emission in metals. To find the potential for core orbital $\alpha \sigma$, they subtracted the self-Coulomb potential $u\left(\left[n_{\alpha \sigma}\right] ; \overrightarrow{\mathrm{r}}\right)$ from the LSD potential (ignoring self-exchange correlation), and remarked that this gave a small correction to the shape of the orbital which translated into a large improvement in the ionization cross section near threshold. From the present study (cf. Fig. 5) it is clear, however, that the exchange and correlation contribution to the self-interaction correction for the eigenvalue [last term in Eq. (39)] is very large and of the opposite sign to the Coulomb term included by Bryant and Mahan.

Stoll, Pavlidou, and Preuss ${ }^{106}$ suggested that the correlation energies of atoms and molecules could be estimated by the equation

$$
\begin{aligned}
E_{c}= & \int d^{3} r n(\overrightarrow{\mathrm{r}}) \epsilon_{c}\left(n_{\uparrow}(\overrightarrow{\mathrm{r}}), n_{\downarrow}(\overrightarrow{\mathrm{r}})\right) \\
& -\sum_{\sigma} \int d^{3} r n_{\sigma}(\overrightarrow{\mathrm{r}}) \epsilon_{c}\left(n_{\sigma}(\overrightarrow{\mathrm{r}}), 0\right) .
\end{aligned}
$$

Here the first term is the LSD approximation for the total correlation, and the next term subtracts out the LSD approximation for parallel-spin correlation. They argued that the correlation between electrons of parallel spin is large in the homogeneous electron gas but not in atoms and molecules. Stoll ${ }^{107}$ has pointed out that Eq. (84) effectively subtracts out the spurious self-correlation, as in the present work.

Shore, Zaremba, Rose, and Sander ${ }^{108}$ applied density-functional theory to the Wigner crystallization of a low-density electron gas. They observed that in the low-density limit $\epsilon_{\mathrm{xc}}\left(n_{\uparrow}, n_{\downarrow}\right)$ $\propto n^{1 / 3}$, and that an appropriate choice of the proportionality constant, slightly different from the standard Wigner value, elminates the self-interaction in LSD for a variety of site-localized orbitals (exponential, Gaussian, etc.)

Zunger and Cohen ${ }^{109}$ applied a self-consistent self-interaction correction, analogous to the present one, to the local-density potential, including correlation, and displayed the improvements in the eigenvalues as well as the valence-electron total energies that result for a number of monovalent metallic atoms. They also proposed that the self-interaction correction can be profitably wedded to the pseudopotential idea: In a simple metal the valence electrons are delocalized and carry no self-interaction, so they can be treated in LSD; the self-interaction corrections to the core orbitals can then be absorbed into a firstprinciples electron-ion pseudopotential. This program has now been implemented. ${ }^{110}$

Perdew ${ }^{111}$ described the present version of selfinteraction correction to LSD, and tested it in non-self-consistent calculations for small atoms. This work revealed the accuracy of the SIC-LSD energy functional itself (not just the one-electron potential), and of the separate exchange and correlation contributions to it.

\section{CONCLUSIONS}

The Hohenberg-Kohn-Sham ${ }^{6,7}$ density-functional theory, as generalized by von Barth and Hedin ${ }^{8}$ and Levy, ${ }^{35}$ is a formally exact prescription for the ground-state energy, electron density, and spin polarization of any many-electron system. Fractional-occupation numbers are allowed in principle, although further work on the exchangecorrelation functional is needed before they can be used in practice. The exact density functional for the energy is self-interaction free, which means in particular that it satisfies Eq. (29). Approximate density functionals [e.g., the LSD of Eq. (27)] can be made to satisfy Eq. (29) by a simple scheme of self-interaction correction (SIC), Eq. (32). Correction of the self-consistent one-electron potential follows naturally from the variational principle. The most straightforward self-consistent formalism incorporating selfinteraction correction is that of Eq. (37). Like the Hartree approximation which it resembles, it is a density-functional theory in the sense of the Hohenberg-Kohn ${ }^{6}$ theorem, although it does not fit into the Kohn-Sham scheme.

In self-interaction-free approximations (such as Hartree, Hartree-Fock, or SIC-LSD), there is an inequality [Eq. (57)] relating the eigenvalue of an occupied orbital to the relaxed energy difference 
upon removal of the electron from this orbital. This inequality is often close to an equality, and amounts to an "extended Koopmans's theorem", less precise but possibly more powerful then the original.

When applied to LSD, the self-interaction correction properly satisfies the sum rule of Eq. (26) for systems without fractional occupation numbers, and improves the shape of the exchangecorrelation hole. We have applied this SIC-LSD scheme in atomic calculations, with results that are far more accurate than those of LSD for a modest increase of computational effort. (In comparison with HF, SIC-LSD is both simpler and more accurate.) Calculations of this type were recently shown $\mathrm{n}^{110}$ to generate accurate a priori pseudopotentials to describe the interaction between valence electrons and ions in more extended systems such as molecules and solids. The SIC scheme can also be applied directly to the allelectron problem in molecules and solids, if care is taken to avoid the "gremlins" discussed in Sec. II F.

Benefits of applying self-interaction correction to the LSD approximation in atomic calculations include: (i) improved values for the total energy and for the separate exchange and correlation pieces of it, (ii) accurate binding energies of negative ions, which otherwise could only be calculated by heavy CI calculations, (iii) orbital eigenvalues that closely approximate physical removal energies, including orbital relaxation effects (in sharp contrast to the LSD eigenvalues, which are almost useless for quantitative purposes), (iv) correct long-range behavior of the one-electron potential and density, and (v) improved overall description of the density. Our calculations also suggest that self-interaction correction will remedy the LSD underestimate of the band gaps in insulators (as shown by numerical results for the rare-gas solids and ionic crystals) and the LSD overestimate of the cohesive energy of the $3 d$ transition metals. However, self-interaction correction is not the cure for all the ills of LSD, and in particular it leaves the spin splitting in $\mathrm{Ni}$ and the $s-d$ interconfigurational energies of the transition elements unimproved and essentially unchanged.

\section{ACKNOWLEDGMENTS}

One of us (J.P.) acknowledges valuable discussions with L. Cole, D. Langreth, J. Rose, H. Shore, and S. Vosko. We are grateful to G. Baraff, L. Hedin, D. Koelling, I. Lindgren, and $\mathrm{H}$. Stoll for pointing out a number of important references to us. Special thanks are due to
D. Ceperley for sending us his numerical data on the electron-gas correlation energies prior to publication, to L. Kleinman for correcting our earlier misconception about the spin splitting in metallic $\mathrm{Ni}$, and to $\mathrm{M}$. Levy for comments on the manuscript and collaboration on Appendix A. One of us (J.P.) acknowledges the support of the National Science Foundation (Grant No. DMR 7812398).

\section{APPENDIX A: COMMENTS ON THE DEFINITION OF THE KINETIC ENERGY}

Here we will present an alternate definition of $T\left[n_{t}, n_{\downarrow}\right]$ which is equivalent to that of Eq. (14). We will then discuss the nature of the "minimization" which appears in the Kohn-Sham formalism of Eq. (17) and in the Hartree-type formalism of Eq. (37).

If the occupation numbers $f_{\alpha \sigma}$ which yield the minimum in Eq. (14) are all 0 or 1 , then the definition (14) obviously reduces to the original definition of Kohn and Sham ${ }^{7}: T\left[n_{\uparrow}, n_{\downarrow}\right]$ is the ground-state kinetic energy of a system of noninteracting electrons with spin densities $n_{\uparrow}(\vec{r})$ and $n_{\downarrow}(\overrightarrow{\mathrm{r}})$. If some of the minimizing occupation numbers are fractional, then Eq. (14) is equivalent to a more general definition. Levy ${ }^{36}$ originally proposed the generalization

$$
T\left[n_{\uparrow}, n_{\downarrow}\right]=\min \langle\hat{T}\rangle,
$$

when $\hat{T}$ is the $N$-electron kinetic-energy operator and the minimum is over all antisymmetric $\mathrm{N}$ electron wave functions which yield the given spin densities. Valone $\mathrm{e}^{112}$ has proposed that the search in Eq. (10) be made over statistical mixtures of antisymmetric $\mathrm{N}$-electron wave functions; if we apply this search also in Eq. (A1), we find that the definitions of Eqs. (14) and (A1) are equivalent, since by Coleman's theorem ${ }^{113}$ the necessary and sufficient conditions for a Hermitian one-particle density matrix $n\left(\overrightarrow{\mathrm{r}}, \overrightarrow{\mathrm{r}}^{\prime}\right)$ to arise from some statistical mixture of $\mathrm{N}$-fermion wave functions are that its eigenvalues (occupation numbers) lie in the interval $0 \leqslant f_{\alpha \sigma} \leqslant 1$, and $\int d^{3} r n(\overrightarrow{\mathrm{r}}, \overrightarrow{\mathrm{r}})=\sum_{\alpha \sigma} f_{\alpha \sigma}=N$. We now consider the nature of the "minimization" in Eq. (14). If the trial orbitals $\psi_{\alpha \sigma}$ are constrained to be orthogonal, then $T\left[n_{\uparrow}, n_{\downarrow}\right]$ is a true minimum of $\sum_{\alpha \sigma} f_{\alpha \sigma}\left\langle\psi_{\alpha \sigma}\left|-\frac{1}{2} \nabla^{2}\right| \psi_{\alpha \sigma}\right\rangle$. Note, however, that the Euler equation (17) is derived without imposing the orthogonality constraint. Thus if the trial orbitals are not constrained to be orthogonal, $T\left[n_{\uparrow}, n_{\downarrow}\right]$ will be the lowest-lying extremum of $\sum_{\alpha \sigma} f_{\alpha \sigma}\left\langle\psi_{\alpha \sigma}\left|-\frac{1}{2} \nabla^{2}\right| \psi_{\alpha \sigma}\right\rangle$ such that no orbital is more than singly occupied, and this extremum is not necessarily a minimum. (It might, for example, be a saddle point.) The extremizing or- 
bitals, being solutions of Eq. (17), are of course orthogonal.

These observations become more than academic in the Hartree-type theory of Sec. II B, when Eq. (14) no longer applies and the extremizing orbitals are no longer orthogonal. In this case, "minimization over the orbitals" really means extremization. However, "minimization over the spin densities" retains its unqualified meaning.

\section{APPENDIX B: FRACTIONAL OCCUPATION NUMBERS}

We need to define the energy functional for fractional occupation for two distinct but related reasons: (1) According to Eq. (54), the relaxed energy difference upon removing an electron from orbital $\alpha \sigma$ is the integral of the orbital eigenvalue $\epsilon_{\alpha \sigma}\left(f_{\alpha \sigma}\right)$ over the interval $0 \leqslant f_{\alpha \sigma} \leqslant 1$. From this point of view, the most natural continuation of $E_{x c}$ is one which satisfies Eq. (29), since in that case the eigenvalue of an isolated orbital over the range $0 \leqslant f_{\alpha \sigma} \leqslant 1$ will be the total energy at full occupancy $\left(f_{\alpha \sigma}=1\right)$. Then for many-electron systems the whole dependence of $\epsilon_{\alpha \sigma}$ on $f_{\alpha \sigma}$ will come from physical relaxation effects. (2) There may be systems in which the energy minimizes with fractional occupation numbers at the Fermi level, so here the continuation of $E_{x c}$ must be a physical one.

Fractional occupation numbers arise whenever an electron hops between degenerate orbitals. Let us consider a model system composed of $N$ protons equally spaced around a ring, and one electron. The solution of the Kohn-Sham equations is a molecular orbital in which the electron is shared equally by all the protons. As the proton separation goes to infinity, one can also define $1 s$ hydrogenic orbitals on each proton which are arbitrarily close to being solutions of the KohnSham equations; these orbitals have fractional occupation $1 / N$. (The electron stays a long time on one proton before hopping to another.) The energy must be the same as it was for an isolated hydrogen atom, which it will be if Eq.(29) is satisfied for fractional occupation.

As a second example, consider two protons and two electrons. For small interproton separations, the Kohn-Sham solution of lowest energy will put both electrons, with opposite spins, into the same molecular orbital. As the separation increases, a point may be reached where a spin-split solution, with the spin-up electron on proton 1 and the spin-down electron on proton 2 , has the lower energy. ${ }^{114}$ This situation is degenerate with one in which the spin-down electron is on proton 1 and the spin-up on proton 2. The physical situation is that the spin on each proton flips up and down, and this can be described by giving half-occupancy to the spin-up and spin-down orbitals on each proton. This situation is not properly described by our scheme of self-interaction correction, which properly eliminates the interaction of each orbital with itself but improperly retains an interaction between the two fractionally occupied orbitals on the same proton.

\section{APPENDIX C: PARAMETRIZED CORRELATION ENERGIES}

Here we describe the parametrization we used for $\epsilon_{c}\left(n_{t}, n_{\downarrow}\right)$, the correlation energy per electron of a homogeneous electron gas, and explain why we believe it is preferable to other parametrizations.

Ceperley ${ }^{41}$ has calculated the energy of a uniform electron gas over a wide range of densities. He used Monte Carlo techniques to sample a correlated wave function for electrons in a finite volume subject to periodic boundary conditions, and extrapolated the energy per electron to infinite volume. Let us define

$$
\begin{aligned}
& n=\left(4 \pi r_{s}^{3} / 3\right)^{-1}, \\
& \zeta=\left(n_{\uparrow}-n_{\downarrow}\right) / n .
\end{aligned}
$$

Then Ceperley's parametrization of the correlation energy for $r_{s} \geqslant 1$ is

$$
\epsilon_{c}^{i}=\gamma_{i} /\left(1+\beta_{1}^{i} \sqrt{r_{s}}+\beta_{2}^{i} r_{s}\right)
$$

where $i=U$ (unpolarized, $\zeta=0$ ) or $P$ (polarized, $\zeta=1$ ). The corresponding correlation potential is

$$
\begin{aligned}
\mu_{c}^{i} & =\left(1-\frac{r_{s}}{3} \frac{d}{d r_{s}}\right) \epsilon_{c}^{i} \\
& =\epsilon_{c}^{i} \frac{\left(1+\frac{7}{6} \beta_{1}^{i} \sqrt{r_{s}}+\frac{4}{3} \beta_{2}^{i} r_{s}\right)}{\left(1+\beta_{1}^{i}{\sqrt{r_{s}}}+\beta_{2}^{i} r_{s}\right)} .
\end{aligned}
$$

For atomic calculations we also need $\epsilon_{c}$ at high densities $\left(r_{s}<1\right)$ and arbitrary polarizations $0 \leqslant \zeta \leqslant 1$. The leading behavior of the high-density expansion is

$$
\epsilon_{c}^{i}=A_{i} \ln r_{s}+B_{i}+C_{i} r_{s} \ln r_{s}+D_{i} r_{s},
$$

and the corresponding correlation potential is

$$
\begin{aligned}
\mu_{c}^{i}= & A_{i} \ln r_{s}+\left(B_{i}-\frac{1}{3} A_{i}\right)+\frac{2}{3} C_{i} r_{s} \ln r_{s} \\
& +\frac{1}{3}\left(2 D_{i}-C_{i}\right) r_{s} .
\end{aligned}
$$

The constants $A_{U}$ and $B_{U}$ are known from the calculation of Gell-Mann and Brueckner, ${ }^{115}$

$$
A_{U}=0.0311, B_{U}=-0.048
$$

(atomic units). To find $A_{P}$ and $B_{P}$, note that in the high-density limit

$$
\epsilon_{c}^{i}=\epsilon_{c}^{i, \mathrm{RPA}}+\epsilon_{c}^{x 2},
$$


where $\epsilon_{c}^{i, \mathrm{R} P A}$ is the correlation energy in the random-phase approximation and

$$
\epsilon_{c}^{x^{2}}=0.023
$$

is the second-order exchange constant (independent of spin polarization). Using Misawa's ${ }^{116}$ RPA scaling relation

$$
\epsilon_{c}^{P, \operatorname{RPA}}\left(r_{s}\right)=\frac{1}{2} \epsilon_{c}^{U, \operatorname{RPA}}\left(r_{s} / 2^{4 / 3}\right),
$$

we find

$$
A_{P}=0.01555, B_{P}=-0.0269 \text {. }
$$

We used Eqs. (C3) and (C4) for $r_{s}>1$, and Eqs. (C5) and (C6) for $r_{s}<1$. The coefficients $C_{i}$ and $D_{i}$ were chosen to match $\epsilon_{c}^{i}$ and $\mu_{c}^{i}$ at $r_{s}=1$, and turned out to be rather small. [This is an interpolation procedure and not a precise determination of the $O\left(r_{s}\right)$ terms in the high-density expansion.]

For intermediate spin polarizations $0<\zeta<1$, we used a standard interpolation formula, first proposed by von Barth and Hedin, ${ }^{8}$ in which the correlation energy has the same polarization dependence as the exchange energy:

$$
\begin{aligned}
\epsilon_{c}\left(r_{s}, \zeta\right)= & \epsilon_{c}^{U}\left(r_{s}\right)+f(\zeta)\left[\epsilon_{c}^{P}\left(r_{s}\right)-\epsilon_{c}^{U}\left(r_{s}\right)\right], \\
\mu_{c}^{\sigma}\left(r_{s}, \zeta\right)= & \mu_{c}^{U}\left(r_{s}\right)+f(\zeta)\left[\mu_{c}^{P}\left(r_{s}\right)-\mu_{c}^{U}\left(r_{s}\right)\right] \\
& +\left[\epsilon_{c}^{P}\left(r_{s}\right)-\epsilon_{c}^{U}\left(r_{s}\right)\right][\operatorname{sgn}(\sigma)-\zeta] \frac{d f}{d \zeta},
\end{aligned}
$$

where

$$
f(\zeta)=\frac{(1+\zeta)^{4 / 3}+(1-\zeta)^{4 / 3}-2}{2^{4 / 3}-2} .
$$

A more realistic interpolation formula, based on the $\zeta$ dependence of the RPA correlation energy, has recently been given by Vosko, Wilk, and Nusair. ${ }^{117}$

The parameters we used are given in Table XII. For the unpolarized electron gas, where Ceperley' $\mathrm{s}^{41}$ calculations were performed for all $r_{s} \geqslant 1$, we used Ceperley's original parameters, but for the polarized gas, where his calculations

TABLE XII. Parameters used to fit the $r_{s}$ dependence of the correlation energy in an unpolarized $(U)$ and polarized $(P)$ uniform electron gas. Values of $A$ and $B$ are given in Eqs. (C7) and (C11). Parameters in a.u.

\begin{tabular}{crrrr}
\hline & \multicolumn{2}{c}{ Ceperley-Alder } & \multicolumn{2}{c}{ Ceperley } \\
Parameter & \multicolumn{1}{c}{$U$} & \multicolumn{1}{c}{$P$} & \multicolumn{1}{c}{$U$} & \multicolumn{1}{c}{$P$} \\
\hline$\gamma$ & -0.1423 & -0.0843 & -0.1471 & -0.0790 \\
$\beta_{1}$ & 1.0529 & 1.3981 & 1.1581 & 1.2520 \\
$\beta_{2}$ & 0.3334 & 0.2611 & 0.3446 & 0.2567 \\
$C$ & 0.0020 & 0.0007 & 0.0014 & 0.0001 \\
$D$ & -0.0116 & -0.0048 & -0.0108 & -0.0046 \\
\hline \hline
\end{tabular}

TABLE XIII. Correlation energy per electron of the unpolarized and polarized uniform electron gas. CA: parametrized Ceperley-Alder, RPA: numerical randomphase approximation. $\epsilon_{c}$ in $\mathrm{eV}$.

\begin{tabular}{rcccc}
\hline \hline & \multicolumn{2}{c}{ Unpolarized } & \multicolumn{2}{c}{ Polarized } \\
\multicolumn{1}{c}{$r_{s}$} & $\mathrm{CA}$ & $\mathrm{RPA}$ & $\mathrm{CA}$ & $\mathrm{RPA}$ \\
\hline 0.01 & -5.21 & -5.82 & -2.68 & -3.32 \\
0.1 & -3.30 & -3.89 & -1.72 & -2.34 \\
0.5 & -2.07 & -2.65 & -1.10 & -1.68 \\
1 & -1.62 & -2.14 & -0.86 & -1.41 \\
2 & -1.23 & -1.68 & -0.66 & -1.15 \\
3 & -1.01 & -1.44 & -0.55 & -1.01 \\
4 & -0.87 & -1.27 & -0.47 & -0.91 \\
5 & -0.77 & -1.16 & -0.42 & -0.84 \\
6 & -0.69 & -1.06 & -0.38 & -0.79 \\
10 & -0.51 & -0.84 & -0.29 & -0.64 \\
20 & -0.31 & -0.58 & -0.19 & -0.47 \\
50 & -0.16 & -0.35 & -0.10 & -0.29 \\
100 & -0.09 & -0.23 & -0.06 & -0.20 \\
\hline \hline
\end{tabular}

were performed only for $r_{s} \geqslant 10$, we adjusted his parameters to give a better match to the highdensity limit. This adjustment successfully predicted the results of the more recent calculation of Ceperley and Alder ${ }^{42}$ for $r_{s} \geqslant 1$.

Ceperley and Alder ${ }^{42}$ have made a stochastic sampling of an exact solution of the Schrödinger equation for the electron gas. Table XII also gives the parameters we have determined by a

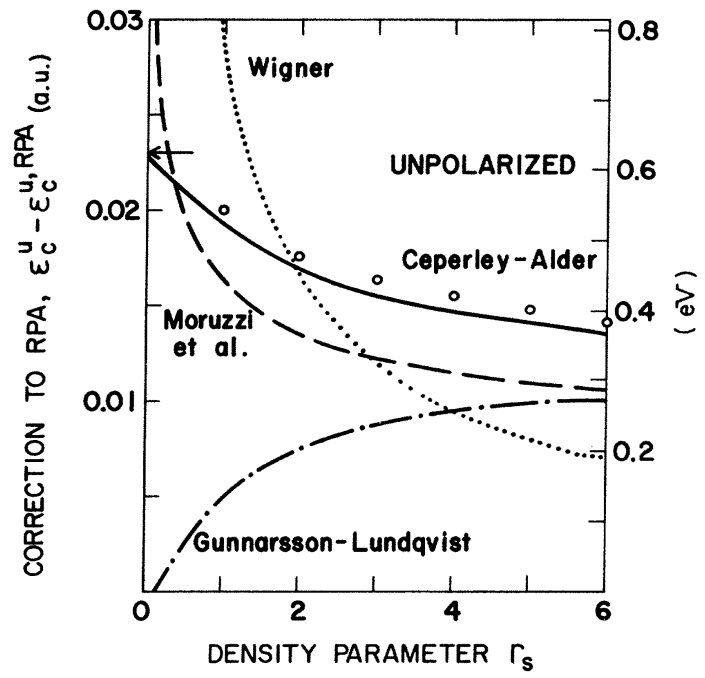

FIG. 13. Correction to the RPA correlation energy of the unpolarized electron gas as a function of $r_{s}$. The parametrized correlation energies considered are those of Ceperley and Alder (Ref. 42, solid curve), Moruzzi, Janak, and Williams (Ref.12, dashed curve), Gunnarsson and Lundqvist (Ref. 40, dash-dotted curve), and Wigner (Ref. 118, dotted curve). Ceperley values (Ref. 41, open circles) are shown for comparison. The arrow shows the exact high-density limit of the correction. 


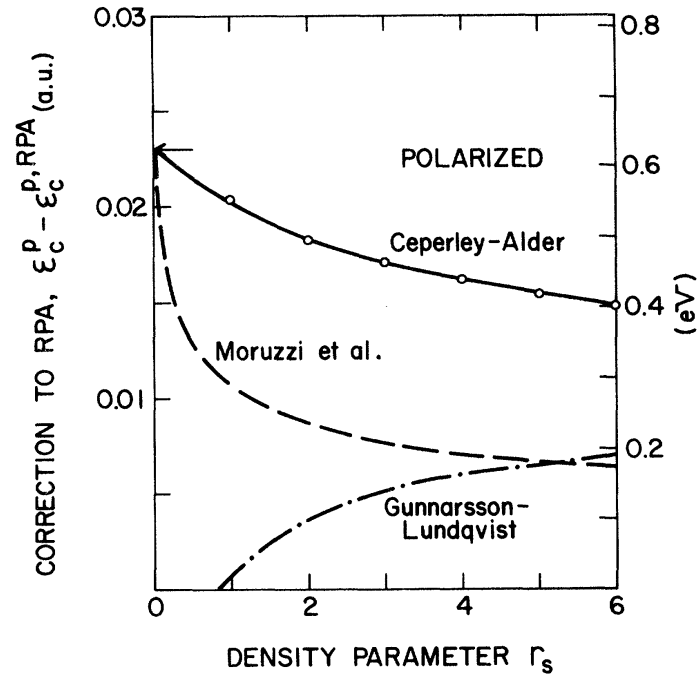

FIG. 14. Same as Fig. 13, but for the polarized electron gas.

careful fit to their numbers. For the densities they considered $\left(r_{s}=1,2,5,10,20,50,100\right)$, our parametrization never deviates from their results by more than 0.0001 a.u. In Table XIII we compare the parametrized Ceperley-Alder correlation energies with numerical RPA correlation energies. ${ }^{117}$ The difference between the Ceperley-Alder values and Ceperley values we used in our calculations can be gleaned from Figs. 13 and 14; although the difference is very small, the Ceperley-Alder values should be preferred in future work. Vosko, Wilk, and Nusair ${ }^{117}$ have independently parametrized the Ceperley-Alder results in a different way, but for the $r_{s}$ values shown in Table XIII their numbers never deviate from ours by more than $1.3 \%$ relatively or by more than 0.0013 a.u. absolutely.

One way to assess the accuracy of the correlation energy is to plot $\epsilon_{c}^{i}-\epsilon_{c}^{i, \mathrm{RPA}}$ vs $r_{s}$, as suggested by Vosko, Wilk, and Nusair. ${ }^{117}$ As $r_{s} \rightarrow 0$, this difference correctly approaches the secondorder exchange constant (C9) in our parametrizations, but not in some other parametrizations that have often been used in density-functional calculations. In Figs. 13 and 14, we compare the high-density behavior of our parametrization of Ceperley-Alder correlation with the parametrizations of Wigner, ${ }^{118}$ Gunnarsson and Lundqvist, ${ }^{40}$ and Moruzzi, Janak, and Williams. ${ }^{12}$ [The latter employs the Hedin-Lundqvist ${ }^{119}$ parametrization of the Singwi et al. calculation ${ }^{120}$ for the unpolarized gas, and then uses the RPA scaling relation (C10) to estimate $\epsilon_{c}$ for the polarized gas.]

Recently Zabolitzky ${ }^{121}$ has obtained a numerical upper bound on the correlation energy of the infinite uniform electron gas; his results for $1<r_{s}$ $<20$ are greater than the Ceperley-Alder ${ }^{42}$ values by about $5 \%$ for the unpolarized gas, and about $15 \%$ for the polarized gas. Thus his results are consistent with the Ceperley-Alder results, and closer to them than to most other calculated correlation energies.
${ }^{1}$ E. Fermi and E. Amaldi, Accad. Ital. Rome 6, 119 (1934).

${ }^{2}$ J. C. Slater and J. H. Wood, Int. J. Quantum Chem. 4, 3 (1971).

${ }^{3} \bar{N}$. W. Ashcroft and N. D. Mermin, Solid State Physics (Holt, Rinehart and Winston, New York, 1976).

${ }^{4}$ A. B. Kunz, Phys. Rev. B 12, 5890 (1975).

${ }^{5} \mathrm{~J}$. C. Slater, The Self-Consistent Field for Molecules and Solids (McGraw-Hill, New York, 1974).

${ }^{6}$ P. Hohenberg and W. Kohn, Phys. Rev. 136, B864 (1964).

${ }^{7}$ W. Kohn and L. J. Sham, Phys. Rev. 140, A1133 (1965).

${ }^{8} \mathrm{U}$. von Barth and L. Hedin, J. Phys. C $\underline{5}, 1629$ (1972).

Also A. K. Rajagopal and J. Callaway, Phys. Rev. B 7, 1912 (1973).

${ }^{9} \mathrm{O}$. Gunnarsson, B. I. Lundqvist, and J. W. Wilkins, Phys. Rev. B 10, 1319 (1974).

${ }^{10} \mathrm{O}$. Gunnarsson, J. Harris, and R. O. Jones, J. Chem. Phys. 67, 3970 (1977).

${ }^{11}$ B. I. Dunlap, J. W. Connolly, and J. R. Sabin, J. Chem. Phys. 71, 4993 (1979).

${ }^{12}$ V. L. Moruzzi, J. F. Janak, and A. R. Williams, Calculated Electronic Properties of Metals (Pergamon, New York, 1978).
${ }^{13}$ O. Gunnarsson, J. Phys. F 6, 587 (1976); J. Callaway, D. Laurent, and C. S. Wang, in Transition Metals, edited by M. J. G. Lee, J. M. Perg, and E. Fawcett (The Institute of Physics, London, 1977), p. 41.

${ }^{14}$ S. H. Vosko, J. P. Perdew, and A. H. MacDonald, Phys. Rev. Lett. 35, 1725 (1975).

${ }^{15}$ A. Zunger and A. J. Freeman, Phys. Rev. B 15, 47 (1977); 15, 5049 (1977); 17, 2030 (1978); Int. J. Quantum Chem. S11, 539 (1977); Ref. 31.

${ }^{16}$ N. D. Lang, Solid State Phys. 28, 225 (1973).

${ }^{17}$ R. Monnier and J. P. Perdew, Phys. Rev. B 17, 2595 (1978); R. Monnier, J. P. Perdew, D. C. Langreth, and J. W. Wilkins, Phys. Rev. B 18, 656 (1978); J. P. Perdew and R. Monnier, J. Phys. F 10, L287 (1980).

${ }^{18} \mathrm{~J}$. A. Appelbaum and D. R. Hamann, Rev. Mod. Phys. 48,3 (1976).

${ }^{19}$ G. P. Kerker, K. M. Ho, and M. L. Cohen, Phys. Rev. Lett. 40, 1593 (1978).

${ }^{20}$ A. Zunger, Phys. Rev. B 22, 959 (1980).

${ }^{21}$ D. C. Langreth and J. P. Perdew, Solid State Commun. 17, 1425 (1975); Phys. Rev. B 15, 2884 (1977).

${ }^{22} \mathrm{D}$. C. Langreth and J. P. Perdew, Solid State Commun. 31, 567 (1979); Phys. Rev. B 21, 5469 (1980).

${ }^{23} \mathrm{~B}$. Y. Tong and L. J. Sham, Phys. Rev, 144, 1 (1966). 
${ }^{24}$ D. J. W. Geldart and M. Rasolt, Phys. Rev. B $\underline{13}, 1477$ (1976).

${ }^{25}$ K. Schwarz, Chem. Phys. Lett. 57, 605 (1978).

${ }^{26}$ H. B. Shore, J. H. Rose, and E. Zaremba, Phys. Rev. B 15, 2858 (1977).

${ }^{27} \mathrm{U}$. Rössler, in Rare Gas Solids, edited by M. L. Klein and J. A. Venables (Academic, New York, 1976), p. 505.

${ }^{28}$ K. Hamrin, G. Johansson, U. Gelius, C. Nordling, and K. Siegbahn, Phys. Scr. 1, 277 (1970).

${ }^{29}$ S. B. Trickey and J. P. Worth, Int. J. Quantum Chem. S11, 529 (1977).

${ }^{30} \mathrm{M}$. Boring, Int. J. Quantum Chem. S8, 451 (1974).

${ }^{31}$ A. Zunger and A. J. Freeman, Phys. Rev. B 16, 2901 (1977) (and references therein); Phys. Lett. $\overline{57 \mathrm{~A}}, 453$ (1976).

${ }^{32}$ D. E. Eastman, F. J. Himpsel, and J. A. Knapp, Phys. Rev. Lett. 44, 95 (1980).

${ }^{33} \mathrm{~J}$. Harris and R. O. Jones, J. Chem. Phys. 68,3316 (1978).

${ }^{34}$ A. Zunger, J. P. Perdew, and G. L. Oliver, Solid State Commun. 34, 933 (1980). In Fig. 1 of this paper, an error occurred in plotting the LSD splitting between the spin-up and spin-down potentials; the correct splitting is 10 times smaller than the one plotted. We would like to thank L. Kleinman and K. Mednick for drawing our attention to this mistake. The exchange splitting in the SIC scheme for magnetic elements is discussed in Sec III $L$ in the present paper.

${ }^{35}$ M. Levy, Proc. Natl. Acad. Sci. USA 76, 6062 (1979).

${ }^{36}$ In Eq. (14) we are pursuing a "hint" in the unpublished first draft of Ref. 35, to the effect that the kinetic energy can be defined as a functional of the density in a way that admits fractional occupation numbers. See also M. Levy, Bull. Am. Phys. Soc. 24 (4), abstract EI16 (1979).

${ }^{37}$ J. F. Janak, Phys. Rev. B 18,7165 (1978).

${ }^{38}$ The remaining constraints $0 \leqslant f_{\alpha \sigma} \leqslant 1$ make Eq. (23) an inequality. A possible objection to relaxing the constraint $\sum_{\alpha \sigma} f_{\alpha \sigma}=N$ is that, although $\boldsymbol{T}, U$, and $n_{\sigma}$ were defined for noninteger numbers of electrons, $E_{\mathrm{xc}}$ was defined at $Q-T-U$ and $Q$ was defined only for integer numbers of electrons. The answer to this objection is that any continuation of $E_{x c}$, differentiable in the range $0 \leqslant f_{\alpha \sigma} \leqslant 1$, will imply Eq. (23).

${ }^{39}$ R. G. Parr, R. A. Donnelly, M. Levy, and W. E. Palke J. Chem. Phys. 68, 3801 (1978); L. J. Bartolotti, S. R. Gadre, and R. G. Parr, J. Am. Chem. Soc. 102, 2945 (1980).

${ }^{40} \mathrm{O}$. Gunnarsson and B. I. Lundqvist, Phys. Rev. B $\underline{13}$, 4274 (1976).

${ }^{41}$ D. M. Ceperley, Phys. Rev. B 18, 3126 (1978).

${ }^{42}$ D. M. Ceperley and B. J. Alder, Phys. Rev. Lett. $\underline{45}$, 566 (1980).

${ }^{43}$ R. S. Gadre, L. J. Bartolotti, and N. C. Handy, J. Chem. Phys. 72, 1034 (1980).

${ }^{44}$ For example, F. E. Harris, Int. J. Quantum Chem. 13, 189 (1978).

${ }^{45} \mathrm{O}$. Gunnarsson, M. Jonson, and B. I. Lundqvist, Phys. Rev. B 20, 3136 (1979).

${ }^{46}$ P. S. Bagus, Phys. Rev. 139, A619 (1965).

${ }^{47}$ A. R. Williams, R. A. de Groot, and C. B. Sommers, J. Chem. Phys. 63,628 (1975).

${ }^{48}$ L. Hedin and A. Johansson, J. Phys. B 2, 1336 (1969).

${ }^{49} \mathrm{M}$. Gopinathan, J. Phys. B 12 , $521(197 \overline{9})$; Phys. Rev.
A 15, 2135 (1977).

${ }^{50}$ M. M. Morrell, R. G. Parr, and M. Levy, J. Chem. Phys. 62, 549 (1975).

${ }^{51}$ Y. Tal, Phys. Rev. A 18, 1781 (1978); 21, $2186(\mathrm{E})$ (1980).

${ }^{52}$ A. K. Theophilou, J. Phys. C 12, 5419 (1979).

${ }^{53}$ J. Katriel, J. Phys. C 13, L375 (1980).

${ }^{54}$ S. M. Valone and J. F. Capitani, Phys. Rev. A $\underline{23}, 2127$ (1981).

${ }^{55}$ W. J. Hunt and W. A. Goddard, Chem. Phys. Lett. $\underline{3}$, 414 (1969).

${ }^{56}$ J. H. Rose and H. B. Shore, Solid State Commun. 17, 327 (1975).

${ }^{57}$ C. Edmiston and K. Ruedenberg, J. Chem. Phys. $\underline{43}$, S97 (1965).

${ }^{58} \mathrm{We}$ are grateful to Lee Cole for performing this calculation.

${ }^{59} \mathrm{C}$. Froese Fischer, The Hartree-Fock Method for Atoms (Wiley, New York, 1977).

${ }^{60}$ L. A. Hemstreet, Phys. Rev. B 11, 2260 (1975).

${ }^{61} \mathrm{~F}$. Herman, J. P. Van Dyke, and I. B. Ortenburger, Phys. Rev. Lett. 22, 807 (1969); F. Herman, I. B. Ortenburger, and J. P. Van Dyke, Int. J. Quantum. Chem. S3, 827 (1970); I. B. Ortenburger and F. Herman, in Computational Methods in Band Theory, edited by P. M. Marcus, J. F. Janak, and A. R. Williams (Plenum, New York, 1971), p. 469.

${ }^{62} \mathrm{~A}$. Veillard and E. Clementi, J. Chem. Phys. $\underline{49}, 2415$ (1968).

${ }^{63}$ B. Y. Tong, Phys. Rev. A 4 , 1375 (1971).

${ }^{64}$ J. P. Perdew, E. R. McMullen, and A. Zunger, Phys. Rev. A (in press).

${ }^{65} \mathrm{M}$. Levy, S. C. Clement, and Y. Tal, in Chemical Applications of Atomic and Molecular Electrostatic Potentials, edited by P. Politzer and D. G. Truhlar (Plenum, New York, 1981) (in press).

${ }^{66} \mathrm{E}$. Clementi and C. Roetti, At. Data Nucl. Data Tables 14, 177 (1974).

${ }^{67}$ L. C. Green, M. M. Mulder, M. N. Lewis, and J. W. Woll, Phys. Rev. 93, 757 (1954).

${ }^{68} \mathrm{H}$. Hotop and W. C. Lineberger, J. Phys. Chem. Ref. Data $\underline{4}, 539$ (1975).

${ }^{69}$ S. Jagannathan, Ph.D. thesis, University of Georgia, Athens, Ga., 1979 (unpublished).

${ }^{70}$ D. W. Smith, S. Jagannathan, and G. S. Handler, Int. J. Quantum Chem. S13, 103 (1979).

${ }^{71}$ C. E. Moore, Natl. Bur. Stand. (U. S.) Ref. Data Ser. 34, 1 (1970).

${ }^{72}$ M. L. Cohen, M. Schlüter, J. R. Chelikowsky, and S. G. Louie, Phys. Rev. B 12, 5575 (1975); J. A. Appelbaum and D. R. Hamann, Phys. Rev. B $\underline{8}, 1977$ (1973).

${ }^{73}$ (a) A. Zunger and M. A. Rather, Chem. Phys. 30, 423 (1978); (b) A. Zunger and M. L. Cohen, Phys. Rev. B 20, 4082 (1979); (c) A. Zunger, J. Vac. Sci. Technol. 16, 1337 (1979).

${ }^{74}$ T. M. Wilson, Int. J. Quantum. Chem. S2, 269 (1968); 3, 757 (1970).

${ }^{75}$ A. Zunger and M. L. Cohen, 'Phys. Rev. B 20,1189 (1979).

${ }^{76}$ A. Zunger and A. J. Freeman, Phys. Rev. B $\underline{17}, 4850$ (1978); B 17, 2030 (1978).

${ }^{77}$ N. F. Mott, Metal-Insulator Transitions (Taylor and Francis, London, 1974), p. 128.

${ }^{78}$ W. P. Menzel, C. C. Lin, D. Fouquet, E. E. Lafon, 
and R. C. Chaney, Phys. Rev. Lett. 30, 1313 (1973); R. C. Chaney, E. E. Lafon, and C. C. Lin, Phys. Rev. B 4, 2734 (1971); G. E. Laramore and A. C. Switendick, ibid. $\underline{7}, 3615$ (1973); D. M. Drost and J. L. Fry, ibid. 5 , 684 (1972).

${ }^{79}$ L. Kleinman and K. Mednick, Phys. Rev. B 20,2487 (1979).

${ }^{80}$ A. Zunger, Phys. Rev. B 21, 4785 (1980); J. Ihm and M. L. Cohen, Solid State Commun. 29, 711 (1979); Phys. Rev. B 21, 1527 (1980); J. P. Worth and S. B. Trickey, ibid. 11, 3658 (1975); A. Zunger and A. J. Freeman, ibid. 15, 5049 (1977); A. Zunger and M. L Cohen, ibid. 19, 568 (1979); Ref. 29.

${ }^{81}$ R. T. Pool, J. Liesegang, R. C. C. Leckey, and J. G. Jenkin, Phys. Rev. B 11, 5190 (1975).

${ }^{82}$ W. Hanke and L. J. Sham, Phys. Rev. Lett. 33, 582 (1974); 43, 387 (1979).

${ }^{83}$ W. Schafer and M. Schrieber, Solid State Commun. 32, 591 (1979).

${ }^{84} \mathrm{M}$. Bennett and J. C. Inkson, J. Phys. C 10, 987 (1977).

${ }^{85}$ E. Wigner and F. Seitz, Phys. Rev. 43, 804 (1933); 46, 509 (1934).

${ }^{86} \mathrm{~A}$. Zunger (unpublished results).

${ }^{87} \mathrm{~J}$. Harris, Int. J. Quantum. Chem. S13, 189 (1979).

${ }^{88} \mathrm{C}$. Kittel, Introduction to Solid State Physics, 5th ed. (Wiley, New York, 1975), p. 74 (from data furnished by L. Brewer).

${ }^{89} \mathrm{~J}$. Harris and R. O. Jones, J. Chem. Phys. $\underline{70}, 830$ (1979).

${ }^{90} \mathrm{O}$. Gunnarsson and R. O. Jones (unpublished).

${ }^{91} \mathrm{O}$. Gunnarsson and R. O. Jones, Phys. Scr. 21, 394 (1980).

${ }^{92}$ I. Lindgren, Int. J. Quantum Chem. 5, 411 (1971).

${ }^{93}$ R. Latter, Phys. Rev. 99, 510 (1955).

${ }^{94}$ F. Herman and S. Skillman, Atomic Structure Calculations (Prentice-Hall, Englewood Cliffs, 1963).

${ }^{95}$ R. D. Cowan, Phys. Rev. 163, 54 (1967).

${ }^{96}$ D. A. Liberman, Phys. Rev. 171,1 (1968).

${ }^{97}$ I. Lindgren and A. Rosén, Technical Report GIPR-034, Institute of Physics, Chalmers Technical University, Göteborg, Sweden (unpublished).
${ }^{98}$ I. Lindgren and K. Schwarz, Phys. Rev. A $\underline{5}, 542$ (1972).

${ }^{99}$ L. Szasz, I. Berrios-Pagan, and G. McGinn, Z. Naturforsch. 309, 1516 (1975).

${ }^{100}$ J. R. Schrieffer, J. Vac. Sci. Technol. 13, 335 (1976).

${ }^{101}$ J. D. Talman and W. F. Shadwick, Phys. Rev. A 14, 36 (1976).

${ }^{102}$ J. D. Talman, P. S. Ganas, and A. E. S. Green, Int. J. Quantum Chem. S13, 67 (1979).

${ }^{103}$ B. H. Brandow, Adv. Phys. 26, 651 (1977).

${ }^{104} \mathrm{~J}$. A. Alonso and L. A. Girifalco, Phys. Rev. B 17, 3735 (1978).

${ }^{105}$ G. W. Bryant and G. D. Mahan, Phys. Rev. B 17,1744 (1978).

${ }^{106}$ H. Stoll, C. M. E. Pavlidou, and H. Preuss, Theoret. Chim. Acta 149, 143 (1978); H. Stoll, E. Golka, and

H. Preuss, ibid. 55, 29 (1980).

${ }^{107} \mathrm{H}$. Stoll, private communication.

${ }^{108}$ H. B. Shore, E. Zaremba, J. H. Rose, and L. Sander, Phys. Rev. B 18, 6506 (1978).

${ }^{109}$ A. Zunger and M. L. Cohen, Phys. Rev. B 18, 5449 (1978).

${ }^{110}$ A. Zunger, Phys. Rev. B 22, 649 (1980).

${ }^{111}$ J. P. Perdew, Chem. Phys. Lett. 64, 127 (1979).

${ }^{112}$ S. M. Valone, J. Chem. Phys. 73, 1344 (1980).

${ }^{113}$ A. J. Coleman, Rev. Mod. Phys. 35, 668 (1963).

${ }^{114} \mathrm{O}$. Gunnarsson, P. Johansson, S. Lundqvist, and

B. I. Lundqvist, Int. J. Quantum Chem. S9, 83 (1975).

${ }^{115}$ M. Gell-Mann and K. A. Brueckner, Phys. Rev. 106, 364 (1957).

${ }^{116}$ S. Misawa, Phys. Rev. 140, A1645 (1965).

${ }^{117}$ S. H. Vosko, L. Wilk, and M. Nusair, Can. J. Phys. $58,1200(1980)$.

${ }^{118} \mathrm{E}$. P. Wigner, Phys. Rev. 46, 1002 (1934); Trans. Faraday Soc. 34, 678 (1938).

${ }^{119} \mathrm{~L}$. Hedin and $\overline{\mathrm{B}}$. I. Lundqvist, J. Phys. C $\underline{4}, 2064$ (1971).

${ }^{120}$ K. S. Singwi, A. Sjölander, M. P. Tosi, and R. H. Land, Phys. Rev. B 1, 1044 (1970).

${ }^{121}$ J. G. Zabolitzky (unpublished). 